\title{
Time Resolution of the COSMo TPC Prototype by
}

\author{
Russell C. J. Woods
}

\author{
A thesis submitted to the \\ Faculty of Graduate and Postdoctoral Affairs \\ in partial fulfilment of the requirements for the degree of \\ Master of Science \\ Ottawa-Carleton Institute for Physics \\ Department of Physics, Carleton University \\ Ottawa, Ontario, Canada
}

May 2011

(C)Russell C. J. Woods, 2011 


$\begin{array}{ll}\begin{array}{l}\text { Library and Archives } \\ \text { Canada }\end{array} & \begin{array}{l}\text { Bibliotheque et } \\ \text { Archives Canada }\end{array} \\ \begin{array}{l}\text { Published Heritage } \\ \text { Branch }\end{array} & \begin{array}{l}\text { Direction du } \\ \text { Patrimoine de l'édition }\end{array} \\ \begin{array}{l}395 \text { Wellington Street } \\ \text { Ottawa ON K1A ON4 } \\ \text { Canada }\end{array} & \begin{array}{l}\text { 395, rue Wellington } \\ \text { Ottawa ON K1A ON4 } \\ \text { Canada }\end{array}\end{array}$

\author{
Your file Votre référence \\ ISBN: 978-0-494-81684-4 \\ Our file Notre référence \\ ISBN: 978-0-494-81684-4
}

NOTICE:

The author has granted a nonexclusive license allowing Library and Archives Canada to reproduce, publish, archive, preserve, conserve, communicate to the public by telecommunication or on the Internet, loan, distribute and sell theses worldwide, for commercial or noncommercial purposes, in microform, paper, electronic and/or any other formats.

The author retains copyright ownership and moral rights in this thesis. Neither the thesis nor substantial extracts from it may be printed or otherwise reproduced without the author's permission.
AVIS:

L'auteur a accordé une licence non exclusive permettant à la Bibliothèque et Archives Canada de reproduire, publier, archiver, sauvegarder, conserver, transmettre au public par télécommunication ou par l'Internet, prêter, distribuer et vendre des thèses partout dans le monde, à des fins commerciales ou autres, sur support microforme, papier, électronique et/ou autres formats.

L'auteur conserve la propriété du droit d'auteur et des droits moraux qui protège cette thèse. $\mathrm{Ni}$ la thèse ni des extraits substantiels de celle-ci ne doivent être imprimés ou autrement reproduits sans son autorisation.
In compliance with the Canadian Privacy Act some supporting forms may have been removed from this thesis.

While these forms may be included in the document page count, their removal does not represent any loss of content from the thesis.
Conformément à la loi canadienne sur la protection de la vie privée, quelques formulaires secondaires ont été enlevés de cette thèse.

Bien que ces formulaires aient inclus dans la pagination, il n'y aura aucun contenu manquant. 


\section{Abstract}

The International Linear Collider (ILC) is a proposed next generation electronpositron collider, deemed critical to supplement Large Hadron Collider (LHC) physics. The International Large Detector (ILD) concept for ILC proposes a Time Projection Chamber (TPC) with Micro-Pattern Gas Detector (MPGD) readout for central tracking. The new MPGD readout concept of charge dispersion can achieve the ILD-TPC transverse resolution goal with fewer readout pads, and has been adapted by the collaboration for further R\&D; however, the time resolution had not been studied. I present here the first such study using a TPC with Micromegas readout, with data from beam tests at KEK and high magnetic field cosmic ray tests at DESY. I find the time resolution at zero drift to be $\epsilon_{\mathrm{L}_{0}}(\mathrm{KEK})=4.1 \pm 0.1$ ns, and $\epsilon_{\mathrm{L}_{0}}(\mathrm{DESY})=7.2 \pm 0.1$ ns. Extrapolation of these measurements yields a resolution of $\epsilon_{\mathrm{L}}(200 \mathrm{~cm}) \approx 1300 \mu m$, which is consistent with ILD-TPC design requirements. 


\section{Acknowledgements}

This work could not have been completed without the help of many friends and colleagues. I would like to thank to my supervisor, Dr. Madhu Dixit, for sharing his extensive knowledge of physics, and for believing in my work. Thanks to Jim Lacey for always providing his unique perspective of problems, and to Stephen Turnbull and Yun-Ha Shin for their patient explanations of analysis code and encouragement. Thanks to Dr. Cliff Hargrove, Dr. Alain Bellerive, and Dr. Paul Johns, who helped me through what I have learned to be the most difficult times of research: the beginning, and the end. Finally, a heartfelt thanks to Mom, Dad, Jenn, Dave, Gran, Gramps, Elizabeth, and the rest of my family for their unwavering support. 


\section{Contents}

$\begin{array}{lll}\text { Abstract } & \text { ii }\end{array}$

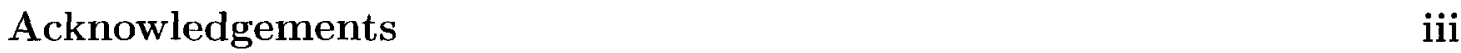

List of Tables vii

List of Figures $\quad$ viii

Abbreviations $\quad x$

1 Introduction 1

1.1 Motivation . . . . . . . . . . . . . . . . . . 1

1.2 The International Linear Collider . . . . . . . . . . . . . . 3

1.3 Resolution Requirements of the ILD . . . . . . . . . . . . . . . 6

1.4 Thesis Outline . . . . . . . . . . . . . . . . . . 7

2 Time Projection Chambers 9

2.1 Fundamentals . . . . . . . . . . . . . . . . . . 10

2.1 .1 Ionization . . . . . . . . . . . . . . . . . 11

2.1 .2 Drift Velocity . . . . . . . . . . . . . . . . 13

2.1 .3 Diffusion . . . . . . . . . . . . . . . . . . 14

2.1 .4 Amplification . . . . . . . . . . . . . . 17

2.1 .5 Readout . . . . . . . . . . . . . . . . . . 19

2.1 .6 Track Reconstruction . . . . . . . . . . . . . . . 20

2.2 Previous TPCs . . . . . . . . . . . . . . . 23

3 Micro Pattern Gas Detector TPCs 24

3.1 Gas Electron Multipliers . . . . . . . . . . . . . . . 25

3.2 Micro-Mesh Gaseous Structures . . . . . . . . . . . . . . 27

3.3 The COSMo TPC . . . . . . . . . . . . . . . . . . . . . . . 29

3.3 .1 Charge Dispersion . . . . . . . . . . . . . 30

3.3 .2 Readout . . . . . . . . . . . . . . . . . 33 


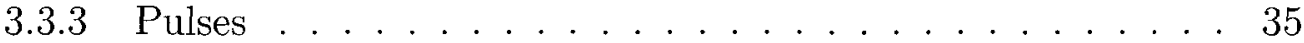

4 Experiments $\quad \mathbf{4 0}$

4.1 KEK Beam Test . . . . . . . . . . . . . . . . . . . . . 40

4.1 KEK Run Summary . . . . . . . . . . . . . . . . . . 42

4.2 DESY High Magnetic Field Test . . . . . . . . . . . . . . . . . . . . 42

4.2 .1 DESY Run Summary . . . . . . . . . . . . . . . 44

4.3 Previous Analysis Results . . . . . . . . . . . . . . . . . . . . 44

5 Time Analysis $\quad 47$

5.1 Pulse Timing . . . . . . . . . . . . . . . . . . . . 47

5.1 .1 Differentiation Method . . . . . . . . . . . . 49

5.1 .2 Error Function Fit Method . . . . . . . . . . . . . . . 52

5.2 Track Fit . . . . . . . . . . . . . . . 55

5.2 .1 Start Time . . . . . . . . . . . . 57

5.2 .2 Drift Velocity .................. 60

5.2 .3 Track Reconstruction . . . . . . . . . . . . . . 61

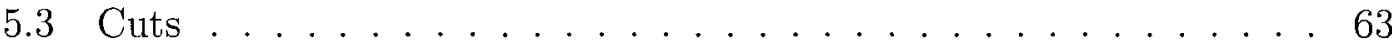

5.3.1 Track Angle . . . . . . . . . . . . . . . . . . . 63

5.3 .2 Edge Events ................... 63

5.3.3 Overflow Pulses . . . . . . . . . . . . . . . 64

5.4 Longitudinal Diffusion Constant . . . . . . . . . . . . . . . . 64

5.5 Resolution ..................... 65

5.5.1 The Geometric Mean Method ............. 66

6 Results $\quad 69$

6.1 Longitudinal Diffusion . . . . . . . . . . . . . . . . . . . 70

6.2 Resolution ..................... 72

6.2 .1 KEK Results . . . . . . . . . . . . . . . . 75

6.2 .2 DESY Results . . . . . . . . . . . . . 76

6.2 .3 Summary . . . . . . . . . . . . . . 77

6.3 Resolution Dependence on $\theta$. . . . . . . . . . . . . 78

7 Conclusion $\quad 81$

A Justification of Linear Track Fit for $(t, \theta) \quad 86$

B Additional Figures $\quad 89$

$\begin{array}{lrr}\text { C Numerical Differentiation } & 97\end{array}$

D Weighted Least-Squares Fit 101 
E Geometric Mean Method 104

$\begin{array}{ll}\text { References } & 108\end{array}$ 


\section{List of Tables}

1.1 Resolution requirements of the ILD-TPC . . . . . . . . 7

4.1 KEK experiment parameters . . . . . . . . . . . . . . . . . 42

4.2 DESY experiment parameters . . . . . . . . . . . . . . 44

5.1 Drift time distribution analysis results. . . . . . . . . . . . 59

5.2 Drift velocity measurement results. . . . . . . . . . . . 61

6.1 Measurements of the intrinsic longitudinal resolution, $\epsilon_{\mathrm{L}_{0}} \ldots \ldots 77$

6.2 Measurements of the effective number of electrons, $N_{\mathrm{eff}} \ldots \ldots \ldots 78$

7.1 Longitudinal resolution achieved by other $\mathrm{TPC}_{\mathrm{s}} \ldots \ldots \ldots$. . . . 84 


\section{List of Figures}

1.1 Proposed design of the International Linear Collider (ILC) . . . . . 4

1.2 Sketch of the International Large Detector concept for the ILC . . . 5

2.1 The basic processes in a wire TPC . . . . . . . . . . 11

2.2 Energy deposited in gas as a function of momentum . . . . . . . . 12

2.3 Avalanche generated on a proportional wire . . . . . . . 18

2.4 Track segment rotation due to $\mathbf{E} \times \mathbf{B}$ effect $\ldots \ldots \ldots \ldots$

2.5 Momentum from track curvature in a magnetic field . . . . . . . . 22

3.1 The Gas Electron Multiplier (GEM) $\ldots \ldots \ldots \ldots$

3.2 Sketch of the Micromegas MPGD . . . . . . . . . . . 28

3.3 Photograph of the COSMo TPC . . . . . . . . . . . 30

3.4 Sketch of an MPGD-TPC readout plane with charge dispersion . . 32

$3.5 \rho(r)$, and $Q(t)$ resulting from charge dispersion . . . . . . 32

3.6 COSMo readout channel map . . . . . . . . . . . . 34

3.7 Typical pulses read out from the COSMo TPC . . . . . . . . 35

3.8 Effect of drift time on readout pulses . . . . . . . . . . . 36

3.9 All 126 pulses readout from a single event in the COSMo TPC . . . 38

3.10 Primary pulses from the 7 readout rows . . . . . . . . . . . 39

4.1 Photograph and sketch of the KEK test apparatus . . . . . . . . . 41

4.2 Photograph and sketch of the DESY experiment setup . . . . . 43

4.3 Distributions of $\phi$ and $x$ for all KEK events . . . . . . . . 45

4.4 KEK transverse resolution plot . . . . . . . . . . . . 45

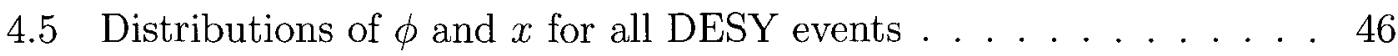

4.6 DESY transverse resolution plot . . . . . . . . . . . 46

5.1 Sketch of drift cluster and pulse formation . . . . . . . . . . 49

5.2 Numerically differentiated pulse fitting . . . . . . . . . . 50

5.3 The effect of baseline noise on pulse differentiation . . . . . . . . . 51

5.4 Estimating parameters for error function pulse fitting . . . . . . 53

5.5 Error function fit to the leading edge of a pulse . . . . . . . . 54

5.6 Comparison of error function fit to numerical differentiation . . . 55

5.7 Linear track fit in the $t-y$ plane $\ldots \ldots \ldots \ldots . \ldots 57$ 
$5.8 \mathrm{KEK} t_{\text {zero }}$ distribution histogram . . . . . . . . . . . . 58

5.9 DESY $t_{\text {zero }}$ distribution histogram . . . . . . . . . . . . . . 59

5.10 KEK reconstructed track parameter distributions . . . . . . . . 62

5.11 DESY reconstructed track parameter distributions . . . . . . . . . . 62

5.12 Calculating inclusive and exclusive residuals . . . . . . . . . . 68

6.1 KEK scatter plot of $\sigma_{\mathrm{L}}$ vs. $z \ldots \ldots \ldots \ldots 71$

6.2 DESY scatter plot of $\sigma_{\mathrm{L}}$ vs. $z \ldots \ldots \ldots 71$

6.3 KEK inclusive and exclusive residual distributions . . . . . . . . . 75

6.4 KEK $\epsilon_{\mathrm{L}}$ vs. $z \ldots \ldots \ldots . \ldots . \ldots . \ldots 75$

6.5 DESY inclusive and exclusive residual distributions . . . . . . . . 76

6.6 DESY $\epsilon_{\mathrm{L}}$ vs. $z \ldots \ldots \ldots \ldots$

6.7 DESY $\epsilon_{\mathrm{L}}$ vs. $z$, extrapolated to ILD-TPC drift distance . . . . . . 77

6.8 DESY $\sigma_{\mathrm{L}}$ vs. $\theta \ldots \ldots \ldots \ldots$

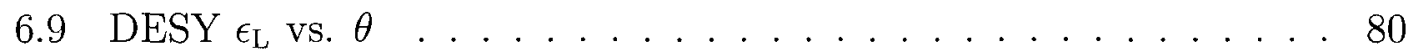

A.1 Forces on a charged particle in a TPC . . . . . . . . 87

B.1 KEK gas parameter curves calculated by Magboltz . . . . . . . . . 90

B.2 DESY gas parameter curves calculated by Magboltz . . . . . . . . . 91

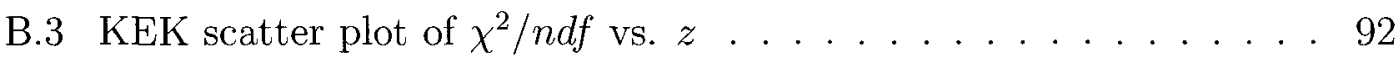

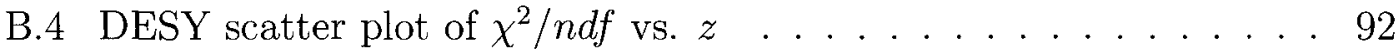

B.5 KEK scatter plot of $\chi^{2} / n d f$ vs. $\sigma_{L} \ldots \ldots . \ldots . \ldots 93$

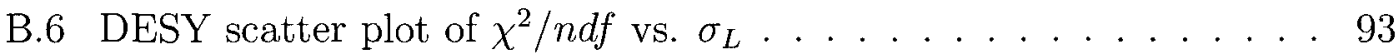

B.7 KEK $\sigma_{L}$ distributions, $0.5 \mathrm{~cm} z$-bins $(z \leq 8 \mathrm{~cm})$, Gaussian fitted . . 94

B.8 KEK $\sigma_{L}$ distributions, $0.5 \mathrm{~cm} z$-bins $(z>8 \mathrm{~cm})$, Gaussian fitted . . 95

B.9 DESY $\sigma_{L}$ distributions, $1 \mathrm{~cm} z$-bins, Gaussian fitted . . . . . . . . 96 


\title{
Abbreviations
}

\author{
ALEPH Apparatus for LEP PHysics
}

ALICE A Large Ion Collider Experiment

BESS Balloon-bourne Experiment with Superconducting Spectrometer

CEA Atomic and Alternative Energy Commission of France.

CERN European Organization for Nuclear Research, Geneva, Switzerland.

COSMo Carleton Orsay Saclay Montreal collaberation

DESY Deutsches Elektronen-SYnchrotron, Hamburg, Germany.

FADC Flash Analogue-to-Digital Converter

GEM Gas Electron Multiplier

ILC International Linear Collider

ILD International Large Detector

JACEE Japanese-American Cooperative Emulsion Experiments

KEK High Energy Accelerator Research Organization, Tsukuba, Japan.

LEP Large Electron Positron Collider, Geneva, Switzerland.

LHC Large Hadron Collider, Geneva, Switzerland.

MPGD Micro Pattern Gas Detector

MWPC Multi-Wire Proportional Chamber

PRF Pad Response Function

RHIC Relativistic Heavy Ion Collider, Brookhaven National Laboratory.

ROOT Object oriented platform for data acquisition, analysis, and simulation.

STAR Solenoidal Tracker At RHIC

T2K Tokai to Kamioka neutrino oscillation experiment, Japan.

TPC Time Projection Chamber

UVTPC University of Victoria Time Projection Chamber 
To break nothing, is to learn nothing.

Götz Ruprecht 


\section{Chapter 1}

\section{Introduction}

\subsection{Motivation}

The Standard Model of Particle Physics is a theory which describes the fundamental building blocks of matter and how they interact with one another, to an extremely high precision. Though the theory has triumphed every time it has been tested by experiment, there still remains one Standard Model particle to be discovered, the so-called Higgs Boson, which would confirm a part of the theory explaining how mass is acquired by fundamental particles.

In March of 2010 the Large Hadron Collider (LHC), the world's largest accelerator, produced two beams of $3.5 \mathrm{TeV}$ protons, and successfully brought them into a head-on collision to produce $7 \mathrm{TeV}$ of centre-of-mass (CM) energy - the highest energy ever achieved in a collider experiment. In a few years it 
is expected to reach $14 \mathrm{TeV}$. With an expected mass of less than $200 \mathrm{GeV}$, the Standard Model Higgs boson should certainly be produced by the LHC if it indeed exists. Its discovery would truly be a monumental event in human history and the crowning achievement of the Standard Model, but it would not foreshadow the end of particle physics. The general consensus in the physics community is that the Standard Model must be a low energy approximation of a deeper theory, one that would also explain mysteries like dark matter, dark energy, matter-antimatter asymmetry, and the unification forces. With the LHC opening up a wide range of terascale centre-of-mass energies, it will allow physicists to weed out proposed theories that do not agree with experiment. However, the LHC is not the best tool for every job.

The precision of the LHC is fundamentally limited by the internal structure of the colliding protons. In the Standard Model, a proton is a baryon, meaning it is composed of 3 quarks bound by the strong force, which is mediated by gluons. The total momentum of the proton is therefore distributed among the quarks and gluons, so that the initial state of a collision between, for example, a gluon from one proton and a gluon from another proton, is not well defined. For this reason it is desirable to collide electrons and positrons (leptons), which are fundamental particles, and thus do have a well defined initial state before a collision.

The problem with an electron-positron synchrotron operating at LHC energies is efficiency. When forced to travel in a curved trajectory, all charged particles radiate energy known as synchrotron radiation. The amount of energy lost per 
revolution, $\Delta \mathrm{E}$, is inversely proportional to the fourth power of the mass of the particle, $m$, and the radius of the synchrotron, $\rho$ :

$$
\Delta E=\frac{1}{\rho}\left(\frac{E}{m}\right)^{4} .
$$

Radiation loss increases rapidly with beam energy, but since an electron is three orders of magnitude lighter than a proton, an electron-positron accelerator of the same size as the LHC would not be able to achieve the same collision energies. In fact, the Large Electron Positron collider (LEP) used the same tunnel as the LHC, but synchrotron radiation limited the achievable collision energy to $209 \mathrm{GeV}$. There are two ways to overcome this problem: (1) build a circular machine with a much larger radius, or (2) forgo the synchrotron in favour of a linear accelerator, which is the more realistic option and chosen by the International Committee for Future Accelerators (ICFA).

\subsection{The International Linear Collider}

The International Linear Collider (ILC), shown in Figure 1.1, is a proposed electronpositron collider that would complement the LHC by making precision measurements of all the Standard Model particles, including the Higgs boson, as well as any new phenomena discovered. The project formed when several regional linear collider efforts, which included Next Linear Collider (NLC) in the USA, the Global 


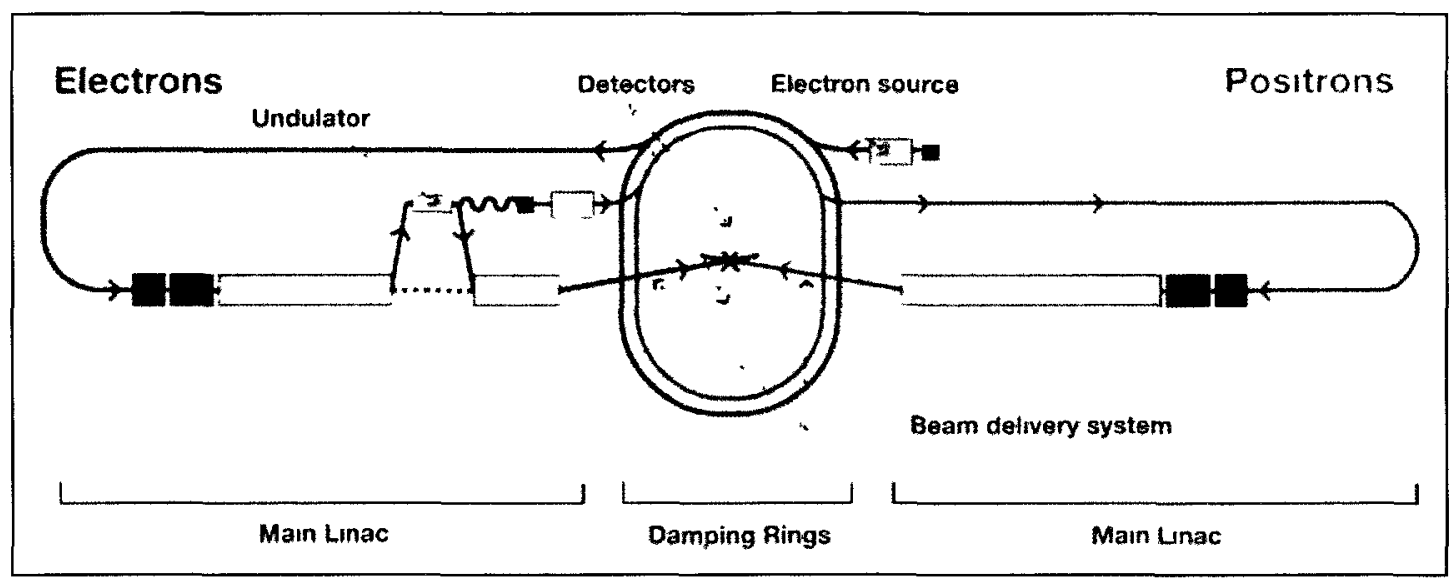

Figure 1.1: Proposed design of the International Linear Collider (ILC), from the 2007 ILC Reference Design Report [1].

Linear Collider (GLC) in Japan, and Tera-electronvolt Energy Superconducting Linear Accelerator (TESLA) in Europe, combined resources to build the ILC using the superconducting RF technology developed for TESLA.

Due to the substantial financial investment required, the status of the project depends on early success at the LHC. The ready-to-construct Engineering Design report should be completed by 2012. It is not expected to deviate significantly from the vision set out by the 2007 ILC Reference Design Report (RDR), which calls for two $15 \mathrm{~km}$ long superconducting linear accelerators to produce 500 $\mathrm{GeV}$ collision energies with a peak luminosity of $2 \times 10^{34} \mathrm{~cm}^{-2} \mathrm{~s}^{-1}$ (which could be upgraded to $1 \mathrm{TeV}$ ).

This ILC would allow for model independent studies of the Higgs boson and related phenomena. The best example of this, the so-called Golden Channel, is the Higgs-strahlung scattering of a $Z$ particle. This process allows for a precise measurement of the Higgs mass, since it is entirely determined by the decay of the 


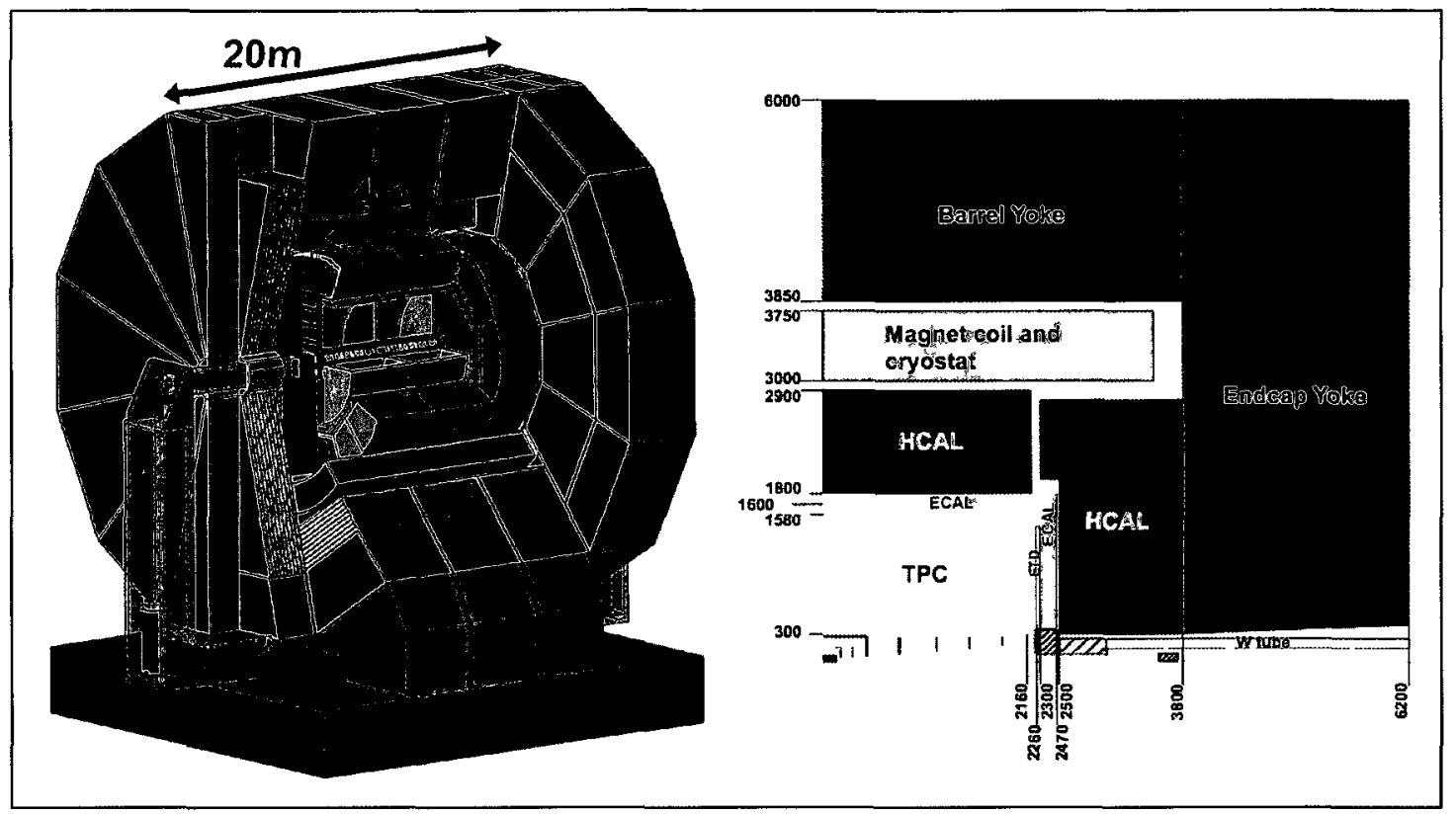

FIgURE 1.2: Sketch of the International Large Detector concept for the ILC, with cross-section showing component details including the Hadronic and Electromagnetic Calorimeters (HCAL and ECAL) from [1].

$Z$ into two leptons, as well as measurements of the Higgs couplings, and searches for invisible decays. The resolution is limited only by how well the collision energy is known, and the precision of the tracker.

Two different detector concepts have been approved to produce Detailed Baseline Design reports by 2012. The most significant difference between the two proposals is how charged particle tracking will be achieved. The Silicon Detector (SiD) concept is based around five layers of silicon detectors to provide a small number of highly accurate track points; while the International Large Detector (ILD) concept, seen in Figure 1.2, proposes a Time Projection Chamber (a gas based detector) for the central charged particle tracker, which is the focus of this thesis. 


\subsection{Resolution Requirements of the ILD}

The required performance of the detectors for the ILC is much greater than at previous accelerators. For example, the event of primary interest for studying the Higgs boson, the so-called golden channel of Higgs production, is the Higgsstrahlung process:

$$
e^{+} e^{-} \rightarrow Z^{0} H \rightarrow l^{+} l^{-} X,
$$

where the collision of a positron $\left(e^{+}\right)$with an electron $\left(e^{-}\right)$results in a $Z$ and Higgs $(H)$ boson. When the $Z$ decays into a lepton, anti-lepton pair, the recoil mass of the Higgs in completely determined from the momenta of the leptons. Therefore, the accuracy of the mass measurement depends only on knowledge of the beam energy and momentum resolution of the two leptons, regardless of the Higgs decay mode $(X)$, and is said to be model independent.

To make the best possible measurement of the Higgs mass, the signal-tonoise ratio (resonance height above background events) needs to be maximized. Monte Carlo studies indicate that the lepton momentum in Equation 1.2 will need to be measured with an accuracy that is 10 times better than what was achieved at LEP [1]. The requirement on the single hit transverse resolution approaches the fundamental limitations imposed by electron statistics, and must be achieved without compromising the longitudinal resolution. The transverse and longitudinal resolution goals for the TPC are summarized in Table 1.1. 
The ILD-TPC will require a large volume ( $4.3 \mathrm{~m}$ long, $3.6 \mathrm{~m}$ diameter) and a small silicon vertex detector at its core surrounding the interaction point. It has been shown that a TPC using Micro-Pattern Gas Detectors (MPGDs) combined with the new concept of charge dispersion, first developed in Canada, can achieve the $100 \mu \mathrm{m}$ transverse resolution goal and reduce detector cost and complexity. However, the longitudinal resolution (in the direction of ion drift) had not been studied.

\begin{tabular}{lll}
\hline Resolution Goals & & \\
\hline Transverse $\left(\epsilon_{\mathrm{T}}\right)$ & $\leq 100 \mu \mathrm{m}$ & $2 \mathrm{~m}$ drift \\
& & \\
Longitudinal $\left(\epsilon_{\mathrm{L}}\right)$ & $1400 \mu \mathrm{m}$ & $2 \mathrm{~m}$ drift \\
& $500 \mu \mathrm{m}$ & zero drift \\
\hline
\end{tabular}

TABLE 1.1: Resolution requirements of the ILD-TPC [2].

\subsection{Thesis Outline}

In this thesis, I present the first study of the longitudinal resolution of a Micromegas TPC with charge dispersion readout, known as COSMo (a collaboration between Carleton, Orsay, Saclay, and Montreal). My responsibilities were to: (1) develop a new timıng algorithm that operates on raw pulses from Flash Analogueto-Digital Converters (FADCs); (2) use data previously collected for studying the transverse resolution to measure the longitudinal resolution with the new algorithm; and (3) compare the results to other TPCs and ILD-TPC requirements given in Table 1.1. 
My thesis proceeds as follows:

- Chapter 2 provides a necessary introduction to the conventional TPC, including the important physical processes, and the limitations on measurement accuracy they impose.

- Chapter 3 covers a modern class of TPC that uses micro-pattern gas detectors (MPGDs), which includes the COSMo prototype, and describes a new readout concept called charge dispersion.

- Chapter 4 describes the two experiments where the data used in this thesis was collected: a high energy $\pi^{+}$beam test conducted in 2005 in Japan, and a high magnetic field cosmic ray test conducted in 2006 in Germany.

- Chapter 5 introduces the different analysis techniques that were used to create the new timing algorithm, and the tests (measurements of known gas parameters) that were used as consistency checks.

- Chapter 6 details the results of the measurements using data from both KEK and DESY: the longitudinal diffusion constant, and longitudinal resolution as a function of both drift distance $(z)$ and track dip angle $(\theta)$.

- Chapter 7 summarizes all results, compares the longitudinal resolution of COSMo with other TPCs and the ILD-TPC goals, and presents some improvements that could be made to the algorithm, as well as other timing methods that could be pursued in the future. 


\section{Chapter 2}

\section{Time Projection Chambers}

First proposed by David R. Nygren at Lawrence Berkeley National Laboratory in the 1970 's to meet the needs of collider experiments with rapidly increasing beam energies, [3], the Time Projection Chamber (TPC) functions like a 3-dimensional high speed camera for charged particles, with close to $4 \pi$ solid angle coverage. The essential property of the TPC which distinguishes it from conventional drift chambers is a magnetic field aligned parallel to the electric field of the drift volume. This has the important advantage of suppressing the transverse diffusion of drift electrons while also functioning as a magnetic spectrometer to allow for the measurement of particle momentum and reconstruction of decay vertices. The amount of ionization collected (the energy loss) allows for the identification of particles. This chapter provides details of how this is all accomplished, as well as physical constraints, and benchmarks of performance set by previous experiments. 


\section{$2.1 \quad$ Fundamentals}

At its most basic, a conventional wire TPC is a gas filled cylindrical chamber, with uniform electric and magnetic fields parallel to the $z$-axis (in cylindrical coordinates), as pictured in Figure 2.1. When a charged particle traverses the gas volume, it ionizes gas molecules leaving a trail of electrons and positively charged ions along its path. The electrons, which have a much greater mobility than the ions, drift and diffuse under the influence of the uniform axial electric and magnetic fields toward the TPC anode where they reach an amplification stage, known as a multi-wire proportional chamber (MWPC). In the vicinity of a wire, the electrons gain enough energy to cause additional ionizations, and thus trigger an avalanche.

Located a few millimetres below each wire is a parallel row of cathode pads that record a capacitively induced signal, providing a means of determining the $\mathrm{x}$ and $\mathrm{y}$ coordinates of the track segment: $y$ given by the wire, and $x$ from the cathode pads (the position along the wire). The track segments, one for each pad row, together form a projection of the original particle track in the $x-y$ plane. Since the drift velocity of the drift electrons is essentially constant, the $z$ coordinate of each track segments is proportional to the drift time of the signal to reach the anode wires. Thus, the TPC can reconstruct the track of a charged particle in 3 dimensions.

To understand the achievable performance, the TPC can be broken down into four basic processes that lead to track reconstruction: ionization, drift and 


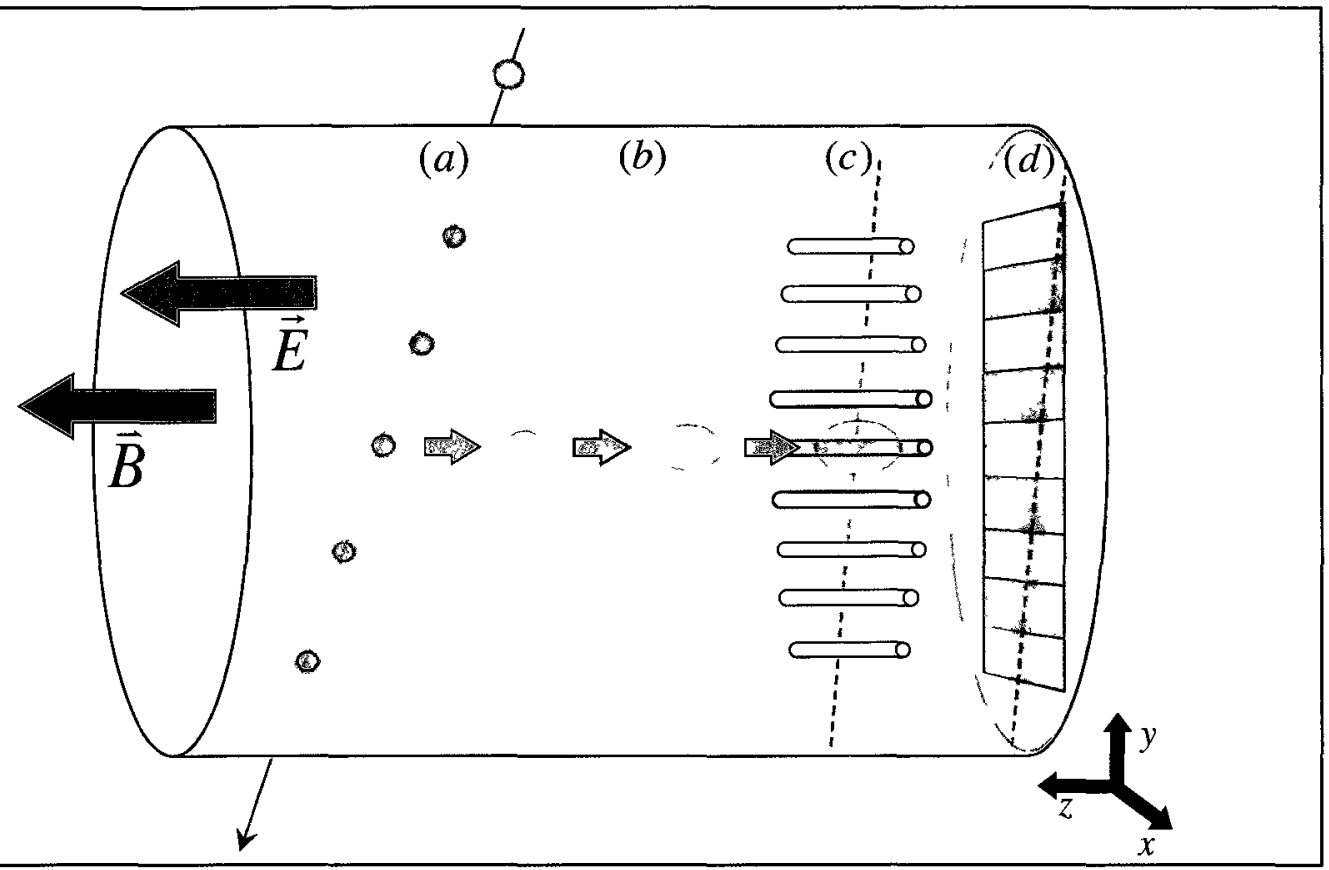

Figure 2.1: The basic processes in a wire TPC: (a) ionization trail, (b) drift and diffusion of electron clusters, (c) amplification of electrons via anode wires, (d) readout of induced signal via cathode pads.

diffusion, signal amplification, and readout.

\subsubsection{Ionization}

When a charged particle passes through the TPC volume, it interacts electromagnetically with gas molecules along its path. The number of these primary interactions is probabilistic, governed by Poisson statistics. The number of electrons (ionizations) resulting from each primary interaction $(0,1,2 \ldots)$ varies, and is referred to as the cluster sıze. The distribution of cluster sizes has a long tail (characterized by a Landau curve [4]), meaning that while typical clusters only contain a few electrons, it is possible they contain many more. This non-uniformity of 


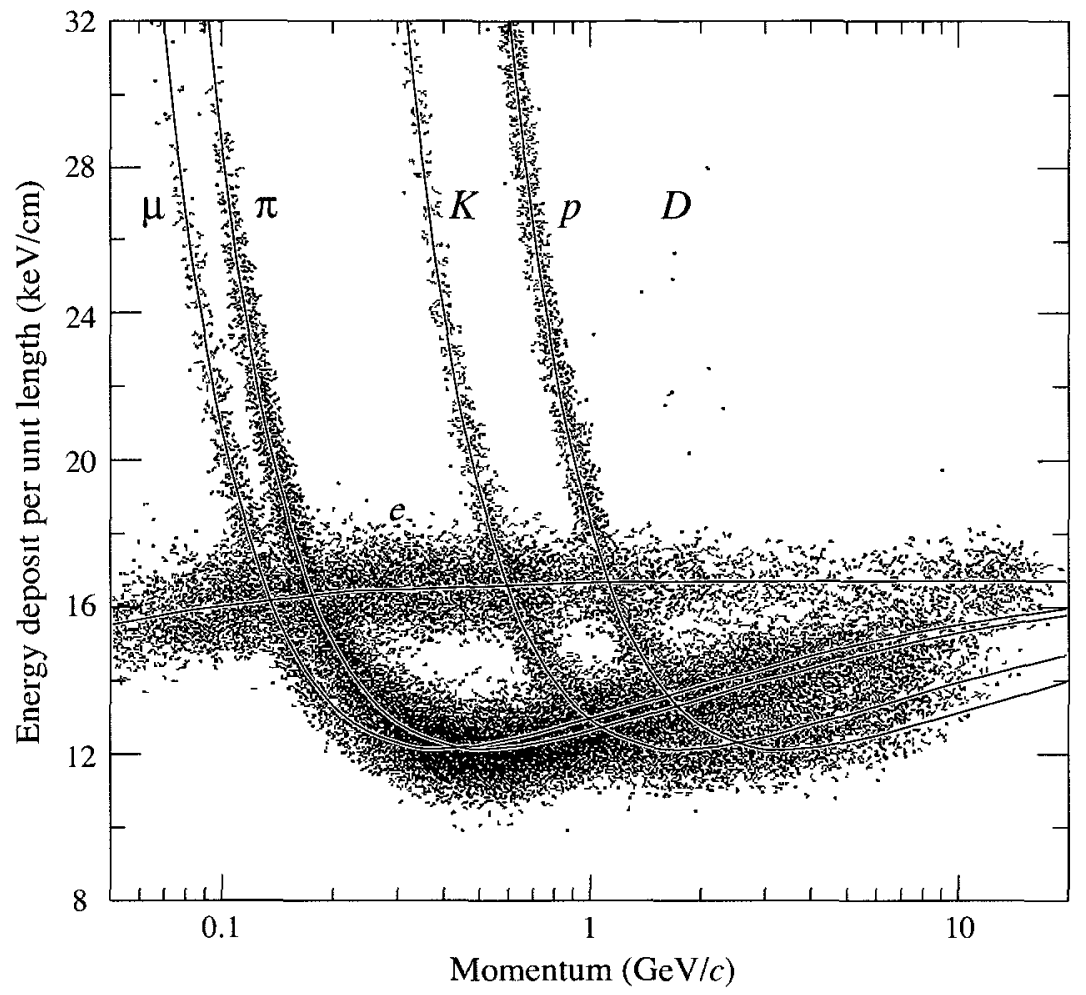

FrguRe 2.2: Energy deposited in gas as a function of ionizing particles momentum, as measured by Nygren's PEP TPC [3].

energy deposited along the track, seen as fluctuations in cluster size, is important because it can potentially worsen resolution by way of the so-called $E \times B$ effect, which will be discussed in Section 2.1.4.

The energy lost by a charged particle moving through a gas per unit length is characterized by the $d E / d X$ curve as a function of momentum. It is also mass dependent, as shown in Figure 2.2. Once momentum is determined from track reconstruction (Section 2.1.6), these well understood energy loss curves (both empirically and from the Bethe-Bloch Equation [5]) can be referenced for particle identification. 


\subsubsection{Drift Velocity}

The electron clusters drift to the readout plane carrying space and time coordinates of the incident particle trajectory because the electric field is uniform throughout the drift volume and parallel to the cylindrical $z$-axis. The uniform field is achieved using conducting field rings placed around the inside of the drift volume, which are connected by a resister chain to step down the voltage from the central membrane cathode of the TPC to the endplate. The electron clusters are accelerated by the electric field, but very quickly reach an equilibrium due to collisions with gas molecules, and drift with a constant velocity, $v_{\mathrm{drift}}$.

The Langevin equation for the drift velocity of an electron in a gas can be expressed as a function of electric and magnetic fields as:

$$
\mathbf{v}_{\mathrm{dr} \mathbf{r f t}}=\frac{\mu}{1+(\omega \tau)^{2}}\left(\mathbf{E}+\frac{\mathbf{E} \times \mathbf{B}}{\mathrm{B}} \omega \tau+\frac{\mathbf{B}(\mathbf{E} \cdot \mathbf{B})}{\mathrm{B}^{2}}(\omega \tau)^{2}\right)
$$

where $\tau$ is the mean time between collisions of the electron with gas molecules, $\mu\left(=e \tau / m_{e}\right)$ is the electron mobility, and $\omega\left(=e B / m_{e}\right)$ is the cyclotron frequency. In an ideal TPC, the electric field $\mathbf{E}$ and magnetic field $\mathbf{B}$ are perfectly parallel, and the equation simplifies greatly to $v_{\text {drift }}=\mu \mathbf{E}$. However, this still depends on one's knowledge of $\tau$ for a particular gas mixture. A commonly used program called Magboltz, written by S. F. Biagi [6], simulates the transport of individual electrons through a chosen gas mixture to predict properties such as drift velocity and diffusion, taking into account using an extensive library of cross-sections. This 
is particularly useful for TPCs that use a gas mixture containing a quencher, as inelastic collisions with these molecules (vibrational and rotational excitations) are much more probable. Drift velocity can also be precisely measured using a UV laser [7], which accounts for any inhomogeneities in the $\mathbf{E}$ and $\mathbf{B}$ fields. Another (albeit more crude) option is to analyze the distribution of drift times, which is explained in Chapter 5 of this thesis.

\subsubsection{Diffusion}

Independent of the average drift velocity, the electrons also have a thermal velocity due to random collisions in the gas, which is given by the Maxwell-Boltzmann distribution and leads to the following equation for the thermal velocity:

$$
v_{\text {thermal }}=\sqrt{\frac{8 k_{\mathrm{b}} T}{\pi m_{\mathrm{e}}}}
$$

where $k_{\mathrm{b}}$ is the Boltzmann constant, $T$ is the gas temperature, and $m_{e}$ is the electron mass. This additional thermal velocity is random in direction (Brownian motion), and is responsible for diffusion. So while the center of gravity of a cluster travels in the axial direction of the TPC with speed $v_{\text {drift }}$, the position of individual electrons obeys a spherical Gaussian probability distribution function spreading in time. For example, the probability distribution function for the $z$ position of a 
single electron is given by:

$$
p(z, t)=\frac{1}{\sigma_{z} \sqrt{2 \pi}} \exp \left[-\frac{1}{2}\left(\frac{z-\mu_{z}}{\sigma_{z}}\right)^{2}\right]
$$

where $\mu_{z}$ is the mean position of the cluster in the $z$ direction, and $\sigma_{z}$ is the width ${ }^{1}$ of the distribution, commonly defined as:

$$
\sigma_{D_{z}}^{2}=D_{z}^{2} t
$$

where $D_{z}$ is the so-called diffusion constant, and carries the dimensions [length] $\times$ $[t \imath m e]^{-\frac{1}{2}}$. However, we find it is more convenient for the width of the charge cluster to depend on drift distance $(z)$ rather than time. Therefore, we choose the diffusion constant to carry units of $\mu \mathrm{m} / \sqrt{\mathrm{cm}}$. In addition, it is particularly convenient in this thesis (as will been seen in Chapter 5) to express the width in units of time rather than distance, therefore we divide by the drift velocity, which gives:

$$
\sigma_{D_{\mathrm{L}}}^{2}=\frac{D_{\mathrm{L}}^{2} z}{v_{\mathrm{drIft}}^{2}}
$$

where the subscript $z$ has been changed to L to emphasize the longitudinal direction. Thus, $\sigma_{D_{\mathrm{L}}}$ can be viewed as the longitudinal diffusion per unit of drift distance, measured in units of time.

\footnotetext{
${ }^{1}$ Throughout this thesis I will refer to the square root of the variance of a distribution as its width.
} 
The same equation can be defined for diffusion in the transverse plane $(x-y)$, however there is a significant difference between diffusion in the transverse plane of a TPC and diffusion in the longitudinal plane. If a drift electron has a velocity component in the transverse plane, then it is moving perpendicular to the direction of the magnetic field, and is subject to the Lorentz force. This causes the electron to spiral in a helical path, reducing the net displacement it can reach in the transverse plane over its drift time, effectively reducing the diffusion by a factor of $\left(1+\omega^{2} \tau^{2}\right)$. Thus, for a given gas mixture, the transverse diffusion constant, $D_{\mathrm{T}}$, depends on both $\mathbf{E}$ and $\mathbf{B}$ fields, whereas longitudinal diffusion only depends on the $\mathbf{E}$ field.

The resolution that can be achieved by a TPC is limited by the width of the clusters at the readout plane, and is therefore dependent on diffusion. Position and time information of the original ionization track, which is carried by the drifting electron clusters, deteriorates with drift distance due to diffusion in the longitudinal plane according to the relationship:

$$
\epsilon_{\mathrm{L}}^{2}=\epsilon_{\mathrm{L}_{0}}^{2}+\frac{\sigma_{D_{\mathrm{L}}}^{2}}{N_{\mathrm{eff}}}
$$

where $\epsilon$ is the single hit longitudinal resolution measured in units of time, $\sigma_{D_{\mathrm{L}}}$ is the cluster width due to diffusion, and $N_{\text {eff }}$ is the effective number of electrons contributing to the measurement. The constant term $\epsilon_{\mathrm{L}_{0}}$ is the zero-drift resolution limit, which is determined by the gas mixture, detector properties, and systematic 
error. It can be thought of as the intrinsic resolution and is therefore used to characterize a detector.

Equivalently, Equation 2.6 can be written for the transverse resolution. Therefore it is important to minimize the transverse diffusion, which is achieved by choosing a gas mixture with a low diffusion constant, coupled with a high magnetic field.

\subsubsection{Amplification}

Amplification is a necessary stage of all gaseous detectors because the number of primary ionization electrons collected by the anode (typically on the scale of a few $\mathrm{mm}$ ) would be too small to be distinguishable from random noise in the electronics. A conventional wire TPC amplifies the ionization using thin anode proportional wires ( $\sim 20 \mu \mathrm{m}$ in diameter) fixed directly above (and parallel to) each row of pads on the readout plane, as illustrated in Figure 2.3.

Anodes are arranged perpendicular to the direction of a typical track. Since the electric field lines must terminate on the wires, the drifting electron clusters follow the converging field lines into the wires. The electric field increases rapidly in the wire region with $1 / r$, and the electrons gain enough kinetic energy to ionize atoms in the gas. The result is a cascading avalanche of electrons in the region very close to the wire. 


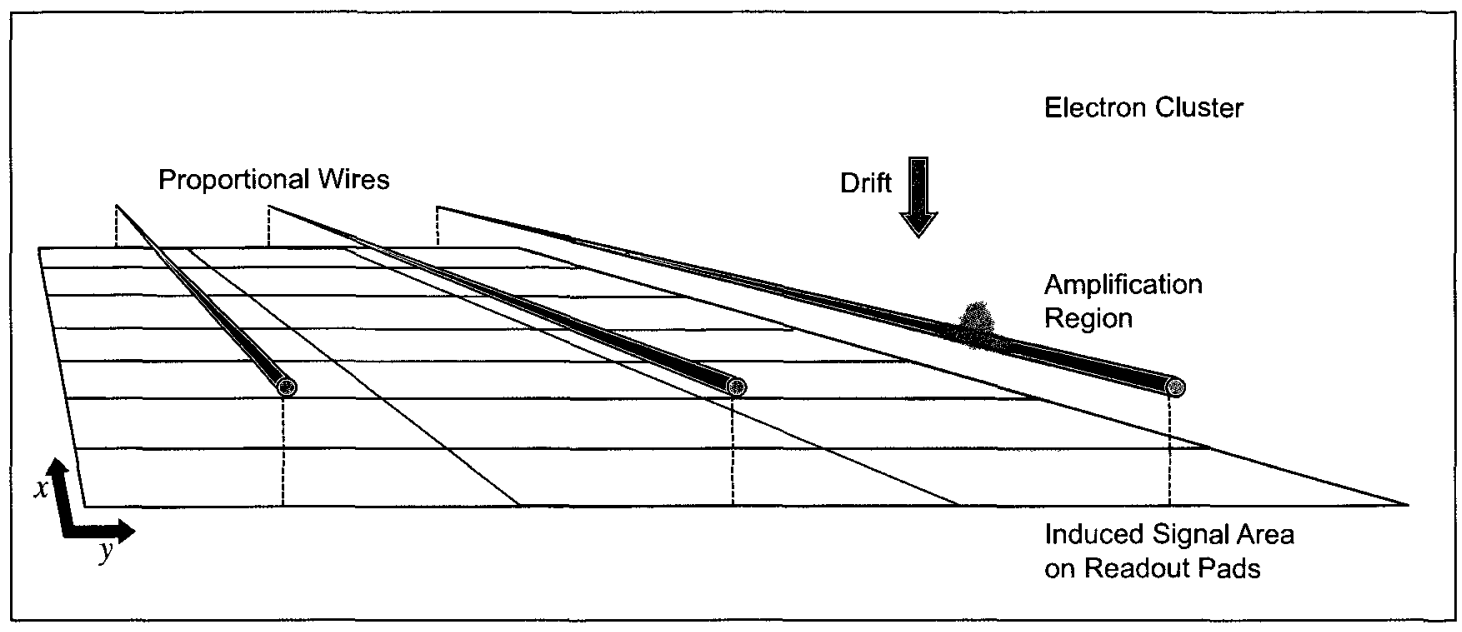

Figure 2.3: Avalanche generated on a proportional wire, and the capacitively induced signal 'area' on the readout pads.

Wire amplification has one unavoidable drawback, commonly known as the $\mathbf{E} \times \mathbf{B}$ effect. As the electric field lines converge to terminate on the anode wires, they are no longer parallel to the $z$-axis near the wire, and therefore no longer parallel to the magnetic field. Therefore the drift electrons gain a component of velocity perpendicular to the magnetic field, and feel a force:

$$
\mathbf{F}_{\mathbf{E} \times \mathbf{B}}=-\mathrm{q} \mathbf{v} \times \mathbf{B},
$$

making them drift normal to both the $\mathbf{E}$ and $\mathbf{B}$ fields. This effectively causes a rotation of the ionization track segment above the wires, as depicted in Figure 2.4. Combined with non-uniform cluster sizes along the ionization track, charge spreading along the wire length leads to an increase in the uncertainty of the hit position as measured by the cathode pads.

The $\mathbf{E} \times \mathbf{B}$ effect is the limiting factor for the spatial resolution which can 


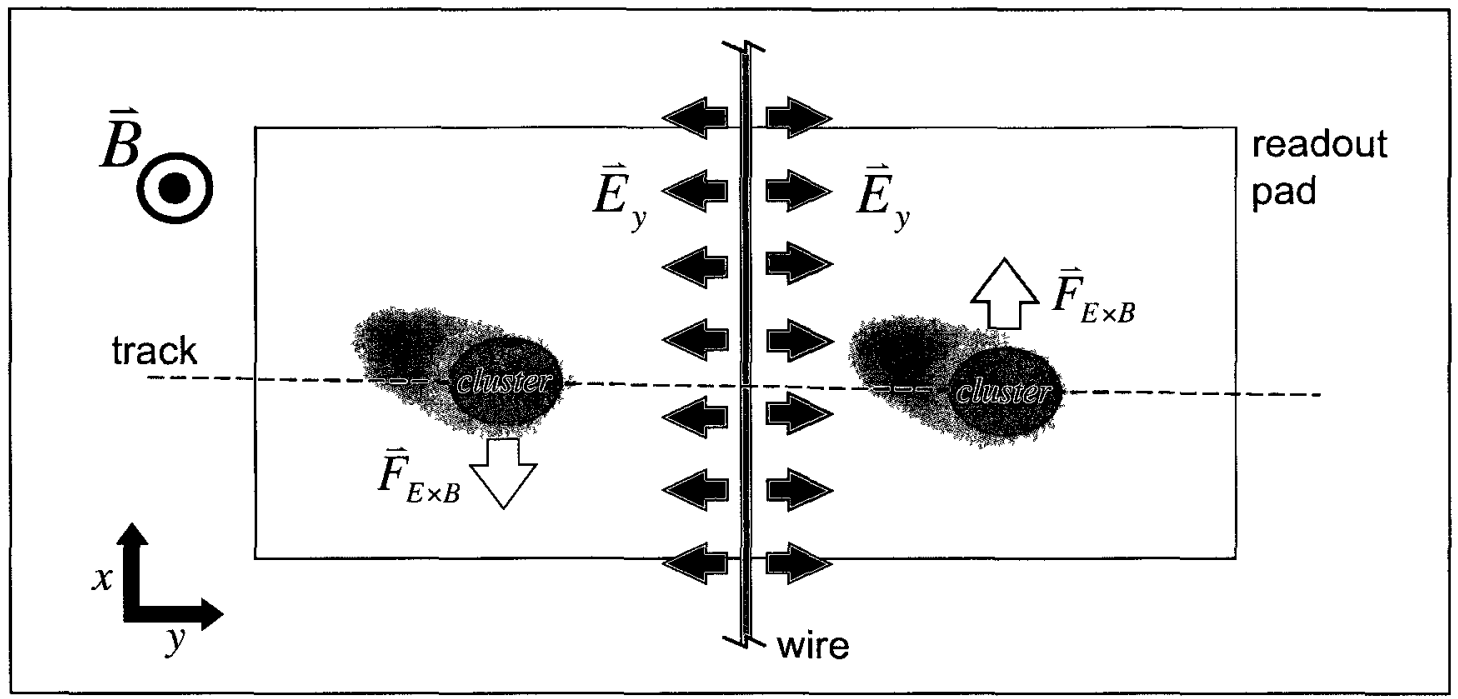

Figure 2.4: Track segment rotation due to $\mathbf{E} \times \mathbf{B}$ effect. If the two clusters have an unequal amount of electrons due to clustering along the track, then the reconstructed track position will be shifted away from the true position.

be attained in conventional time projection chambers. To achieve the resolution goals of the ILC-ILD TPC, a new class of amplification device, know as Micro Pattern Gaseous Detectors (MPGD's), will be used and is discussed in detail in Chapter 3 of this thesis.

\subsubsection{Readout}

In a conventional TPC where proportional wires are used for amplification, it is actually the slow drifting positive ions created in the avalanche, and not the fast moving electron avalanche, that generates a signal in the readout electronics. As positive ions follow the electric field lines away from the wire, they induce a signal current in the anode wire. The cathode pads see the capacitive signal which must 
be amplified using pre-amplifiers. The resulting signal is digitized using Flash Analogue-to-Digital Converters (FADC's) and stored for analysis.

The readout plane of a large wire TPC is actually broken into sectors like slices of a pie, as in the STAR TPC [21]. Since the wires must be straight, and the end plate of TPC is circular, dividing the plane into sectors allows the wires to be approximately perpendicular to tracks originating at the interaction point. The readout pads are arranged in circular rows, and parallel to the high momentum tracks.

The number of readout pads, and hence their dimensions, are chosen as a balance between the resolution goal for the detector, and the budget constraints for the electronic readout. As high momentum tracks are approximately in the radial direction, the most important factor in track reconstruction is the transverse resolution. For this reason, the chosen pad width tends to be significantly smaller than the radial length. The height of the wire above the cathode pads is chosen to ensure that the induced signal from a cluster registers on more than one pad. This is required to achieve sufficient transverse resolution, and is a critical topic in TPC design and will be a recurring theme throughout the rest of the thesis.

\subsubsection{Track Reconstruction}

The track of a particle through the TPC volume, and its momentum, is reconstructed from a finite number of $(x, y, z)$ points, determined by the number of pad 
rows. For example, in our simple TPC displayed in Figure 2.1, there are 9 pad rows, and therefore the track is reconstructed from 9 points. The digitized signals recorded for each cathode pad in a row together provide one point on the projected track in $(x, y)$. This is accomplished through the use of a Pad Response Function (PRF).

The PRF, determined a priori, gives the expected signal amplitude as a function of cluster distance from the center of the $\mathrm{pad}^{2}$. Thus the signal amplitude from each pad in a particular row, together with the PRF, is enough information to calculate the $x$ coordinate. The $y$ coordinate is taken as the row position.

The $z$ coordinate of the track above the anode wire is determined from the time of arrival of signal, $t_{\text {zero }}$ read directly off the anode wire. Since the velocity of clusters through the drift region of the TPC is constant, the original position of the cluster is simply:

$$
z=v_{\text {drift }}\left(t_{\text {zero }}-t_{\text {start }}\right)
$$

where $t_{\text {start }}$ is the time that the original particle passes through the TPC, provided by a fast external trigger.

In general, a helix is fit to the $(x, y, z)$ track points simultaneously. The track momentum can be calculated from the radius of curvature of the projected

\footnotetext{
${ }^{2}$ In the conventional MWPC readout, the PRF takes the form of a simple Gaussian.
} 


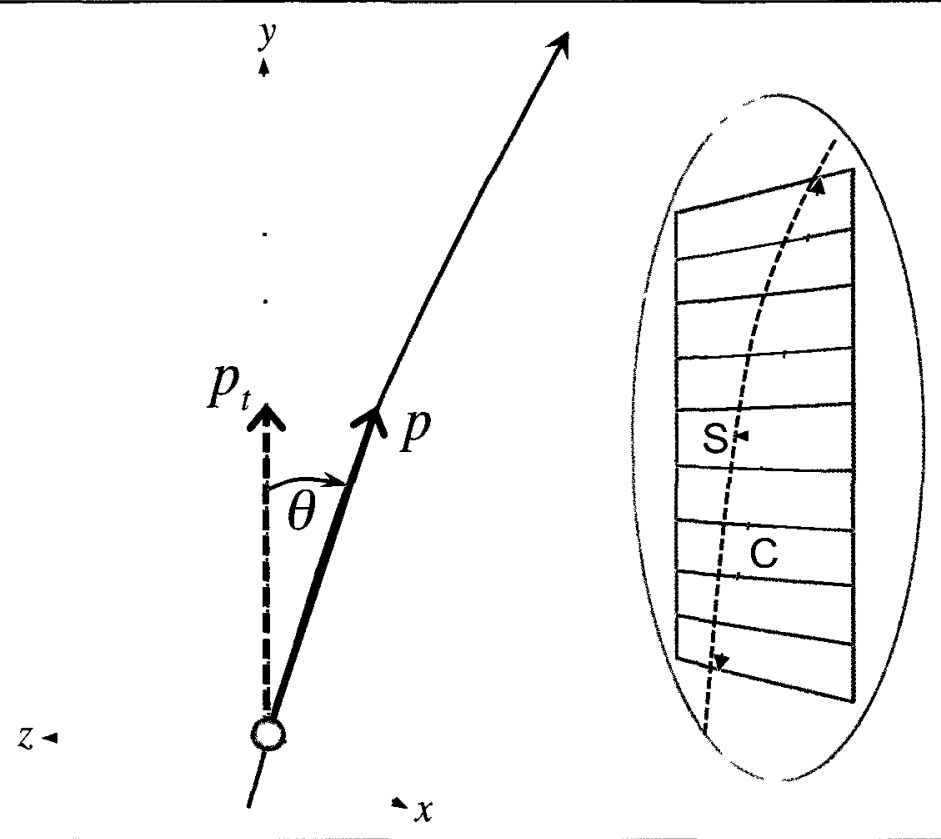

FigURE 2.5: Transverse momentum is determined by the curvature of projected track in the readout plane, which is calculated from the chord length (C) and sagitta (S). Total momentum requires a measurement of $\theta$.

track in the readout plane, $\mathrm{R}$, and the angle $\theta$ :

$$
|p|=\frac{q B R}{\cos \theta}
$$

where $B$ is the magnetic field strength. The momentum resolution therefore depends on the uncertainty of $\mathrm{R}$, which depends on the transverse resolution, and of $\theta$, which depends on the longitudinal resolution.

This thesis is primarily focused on measuring the longitudinal resolution, which is the detector's ability to reconstruct $z$ in Equation 2.8, and $\theta$ in Equatıon 2.9 independently of $x$. Therefore, rather than helical, I wlll focus on linear track fits in $(z(t), y)$, as will be explained with greater detall in Chapter 5. 


\subsection{Previous TPCs}

An important benchmark in conventional TPC performance was the very successful ALEPH experiment [7], which operated in the Large Electron Positron (LEP) synchrotron at CERN and made precision measurements of the $W$ and $Z$ bosons. It operated over the full lifetime of LEP, from 1989 until the decommissioning in 2000 to make way for the LHC.

The ALEPH detector TPC had a diameter of $3.6 \mathrm{~m}$ and a length of $4.4 \mathrm{~m}$, which was the largest at the time and very close to the dimensions of the proposed ILD TPC. The ALEPH TPC used a gas mixture of $91 \%$ argon and $9 \%$ methane, a magnetic field of $1.5 \mathrm{~T}$, and a drift field of $125 \mathrm{~V} / \mathrm{cm}$. The average single hit point resolution achieved was $173 \mu \mathrm{m}$ in the transverse direction, and $740 \mu \mathrm{m}$ in the longitudinal direction (though it should be noted that these values are for stiff high energy $Z^{0}$ decays to two leptons, which are the easiest to measure).

Today, there are two important large scale TPCs being used in high energy heavy ion collider experiments: the STAR detector has been observing $\sim 100 \mathrm{GeV}$ per nucleon collisions of heavy nuclei in RHIC at Brookhaven, and ALICE in the LHC at CERN recently begun studying the collision of $2.76 \mathrm{TeV}$ per nucleon lead ions [5]. The obvious complexity in the aftermath of such collisions speaks to the robust nature of the TPC: a powerful tool for high energy physics. 


\section{Chapter 3}

\section{Micro Pattern Gas Detector}

\section{TPCs}

Conventional TPC's using proportional wires have been proven in many important experiments, and continue to be the right tool for many high energy experiments today. However, the ILD-TPC will need to achieve an order of magnitude better momentum resolution than what is allowed by the intrinsic limitations of wires. Improved techniques of micro-fabrication have led to the development of a new class of amplification devices called Micro-Pattern Gas Detectors (MPGD) which eliminate the $\mathbf{E} \times \mathbf{B}$ problem, suppress ion feedback into the drift volume, and have a low material budget. Two leading candidate MPGD's for the ILD-TPC are the GEM and Micromegas, which have shown excellent potential in smaller scale detectors. 


\subsection{Gas Electron Multipliers}

The Gas Electron Multiplier (GEM), introduced in 1997 by F. Sauli [8], is a widely used MPGD in smaller drift chambers and prototype TPCs. The basic device consists of an insulating dielectric film (typically kapton) coated on both sides with a thin layer of copper, so that the top and bottom layers can maintain a large potential difference without breakdown. The sandwich is perforated with holes which have a typical diameter of $\sim 70 \mu \mathrm{m}$, and are on a $\sim 140 \mu \mathrm{m}$ hexagonal grid. With a few hundred volts applied between the two sides of the device, a high electric field is generated within the holes, typically on the order of $50 \mathrm{kV} / \mathrm{cm}$. Drifting electrons follow converging electric field lines into the GEM holes, where they are rapidly accelerated by the high field causing additional ionization of gas molecules. This results in an avalanche of electrons in the hole region, which exits the other side of the device.

The small distance between holes ensures that drift electrons, no matter where they are generated, will not gain a significant transverse velocity entering a hole. This is important because as explained in Chapter 2, transverse velocity leads to a Lorentz force due to the uniform magnetic field, and fundamentally limits the achievable resolution. Therefore, the resolution of a GEM TPC is not limited by the $\mathbf{E} \times \mathbf{B}$ effect.

To lower the probability of discharge, which can cause detrimental damage to a GEM, it is desirable to keep the voltage across the device as low as possible. 
To achieve the required gain using lower operating voltages, additional GEM's need to be added in series so that the amplification is spread out over multiple stages. While this is generally seen as a disadvantage as it adds complexity to the detector, there is an important benefit. The lower $\mathbf{E}$ field between GEM stages increases the transverse diffusion, so-called defocusing, of the cluster, allowing for the use of larger readout pads. The resolution is not adversely affected because the larger cluster width is balanced by the increase in the number of electrons.

Use of the GEM has been successfully demonstrated in many prototype MPGD TPCs, including the UVic TPC (UVTPC), which was able to achieve good transverse resolution using $1.2 \mathrm{~mm}$ wide pads [10]. However, to cover the $10 \mathrm{~m}^{2}$ ILD-TPC endplate would require approximately 3 million such pads, with an equal number of readout channels, increasing the complexity and heat load (which becomes difficult to remove). For comparison, the ALEPH TPC, with

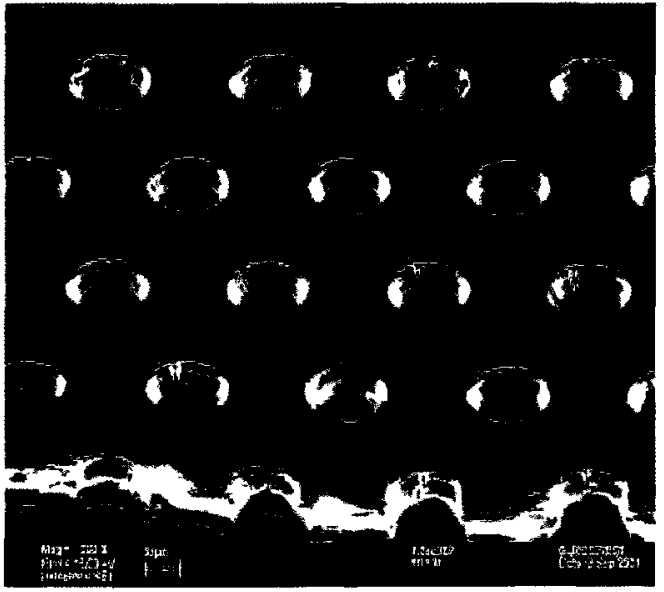

(a)

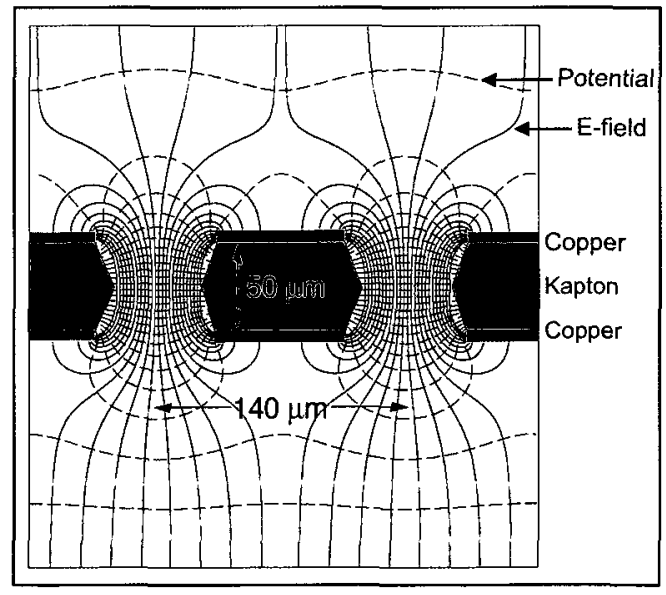

(b)

Figure 3.1: The Gas Electron Multiplier (GEM): (a) 100x magnification image and (b) field map of the hole region, where the Electric field lines are solid (red) and lines of constant potential are dashed (blue), from [9]. 
endplates of approximately the same size, had just under 50,000 readout channels! It is desirable to keep the detector complexity to a minimum, so all options are researched.

\subsection{Micro-Mesh Gaseous Structures}

The Micro-Mesh Gaseous Structure (Micromegas) was introduced in 1995 by I. Giomataris, [11], and is the offspring of the parallel-plate avalanche counter. Unlike the GEM, the Micromegas is physically coupled to the readout plane forming a single device. As shown in Figure 3.2, it consists of a thin $(\sim 3 \mu \mathrm{m})$ conducting mesh with $\sim 50 \mu \mathrm{m}$ pitch, supported by an array of pillars $\sim 100 \mu \mathrm{m}$ in diameter (with $\sim \mathrm{mm}$ pitch) above the anode. A modest voltage difference between the mesh and anode plane divides the gas volume of the detector into a low field drift region, and a high field amplification gap. The width of the amplification gap of the Micromegas is chosen to maximize gain for desirable operating voltages and gas mixtures. An important result is that small non-uniformities in the mesh height and fluctuations in pressure do not cause appreciable variations in gain, as shown in [11].

When an electron drifts through the mesh into the high field region, it gains enough energy to generate an avalanche of electrons and ions, the majority of which are produced very close to the anode. The short distance to the anode means that the signal produced from the electrons is small and too fast to be 


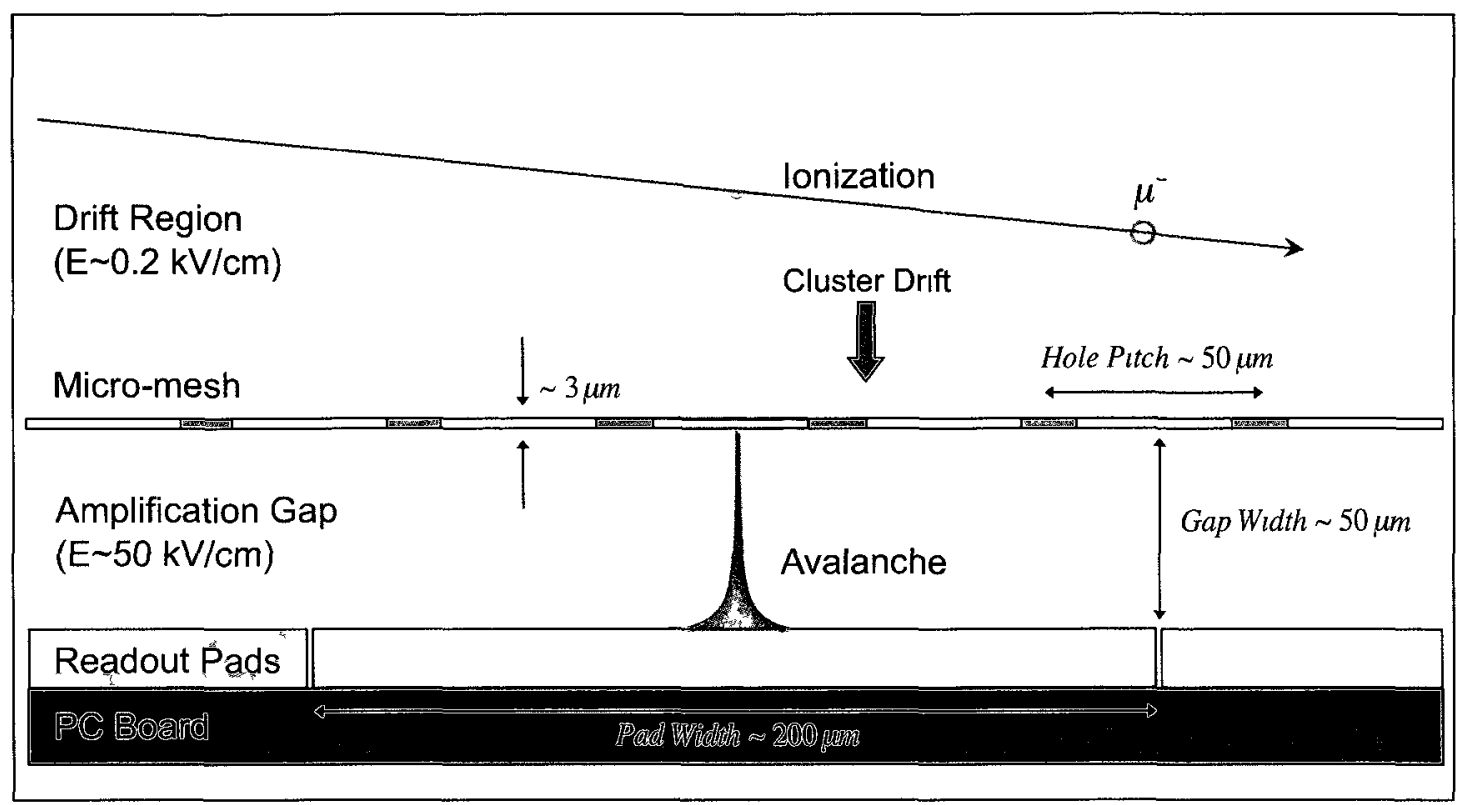

FIGURE 3.2: Sketch of the working principles and dimensions of a Micromegas MPGD in a Time Projection Chamber.

useful. Instead, it is the much larger signal from the slower moving positive ions, which drift back to the mesh across the full amplification gap, that is used for track reconstruction.

The relatively simple construction of the Micromegas device makes it extremely robust. Whereas the GEM is susceptible to defects caused by sparking, the Micromegas have been shown to withstand sparks without loss of performance [12]. Other important advantages of using the device are: the elimination of the $\mathbf{E} \times \mathbf{B}$ effect, and suppression of ion feedback. As with the GEM, the micro-scale distance between holes greatly reduces distance over which the $\mathbf{E}$ field lines converge compared to the conventional anode wire. The mesh also acts as a barrier to positive ions generated in the avalanche from accumulating in the drift volume, which can cause local distortions in the electric field. Conventional TPCs combat 
this problem using a gating grid of wires to absorb positive ions, which may prove unnecessary in a large scale MPGD TPC (this still requires study).

A significant drawback of the Micromegas is that due to the small amplification gap, there is very little diffusion in this region, so much narrower pads are required for charge sharing, as compared to the multiple GEM stage TPC. Whereas the conventional ALEPH TPC was able to achieve excellent resolution with $6 \mathrm{~mm}$ wide pads, to achieve the ILD resolution goal using Micromegas would require $200-400 \mu \mathrm{m}$ wide pads [13]. This has not been a problem for small detectors which have much less readout area to cover, however it would be unreasonable for the $9 \mathrm{~m}^{2}$ ILD-TPC endplates.

\subsection{The COSMo TPC}

A collaboration between Carleton University, Orsay, CEA Saclay, and Université de Montréal (COSMo) formed with the purpose of testing a new Canadian concept for readout, which would make the use of MPGDs in large scale time projection chambers much more feasible by reducing the required number of readout channels needed to achieve the resolution goals of the ILD. The prototype is a small MPGD TPC, shown in Figure 3.3, has a $15.7 \mathrm{~cm}$ drift region, and a $10 \times 10 \mathrm{~cm}^{2}, 50 \mu \mathrm{m}$ gap Micromegas amplification stage. What makes this TPC different from other prototype MPGD TPC's is the inclusion of a resistive anode plane, which improves 


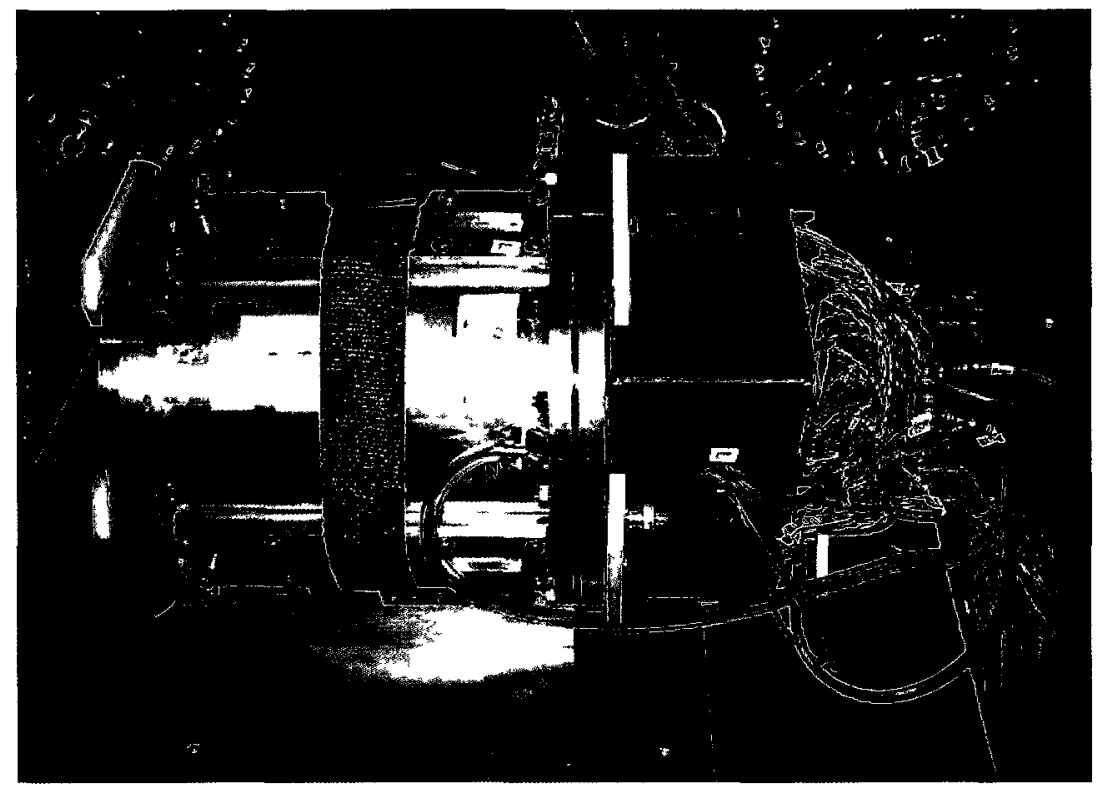

FIGURE 3.3: Photograph of the COSMo TPC.

signal spread over multiple pads, a concept known as charge dispersion that has the potential to achieve ILD-TPC resolution without resorting to narrow pads.

\subsubsection{Charge Dispersion}

To achieve charge dispersion, the conventional MPGD anode plane is modified with a high surface resistivity foil. It is laminated to the readout pads with a thin layer of insulating adhesive $(\sim 50 \mu \mathrm{m})$ which acts as an dielectric spacer, as shown in Figure 3.4. Together with the resistive foil above and readout pads below, this forms a distributed 2-dimensional RC network. Avalanche charge on the resistive anode obeys the 2-dimensional diffusion equation, written as:

$$
\frac{\partial \rho}{\partial t}=\frac{1}{R C}\left(\frac{\partial^{2} \rho}{\partial r^{2}}+\frac{1}{r} \frac{\partial \rho}{\partial r}\right)
$$


where $R C$ is the time constant of the system $(=\tau) ; R$ being the resistivity of the foil and $C$ the capacitance density per unit area of the insulating adhesive [14]. The result is that when a charge avalanche arrives at the surface, it disperses across the plane, covering a larger area with time. The solution of Equation 3.1 is a 2-dimensional Fourier series. This, however, can be simplified using the approximation that the charge deposited is a delta function and the pad edges are at infinity, to:

$$
\rho(r, t)=\frac{R C}{2 t} \exp \left(-r^{2} R C / 4 t\right)
$$

Equation 3.2 is plotted in Figure 3.5, which shows the time evolution of an initially localized surface charge distribution spreading over multiple pads. As this occurs, the electron density on the resistive foil is capacitively mirrored on the readout pads below. Unlike diffusion, charge dispersion does not degrade the resolution, because it is not a statistical process; the spreading of avalanche charge over the surface of the resistive foil is entirely determined by the physical properties of the readout.

The resistive MPGD anode readout is a Canadian concept that was initially conceived at Carleton, and was first tested using an X-ray tube and small drift chamber. It has since been tested with both GEM and Micromegas MPGDs, and is currently studied by many different groups internationally. The COSMo TPC used $\mathrm{a} \sim 1 \mathrm{M} \Omega / \square$ Cermet $(\mathrm{Al}-\mathrm{Si}$ ) coated $25 \mu \mathrm{m}$ Mylar film for both KEK and DESY tests (discussed in Chapter 4). Other options are being tested with the 


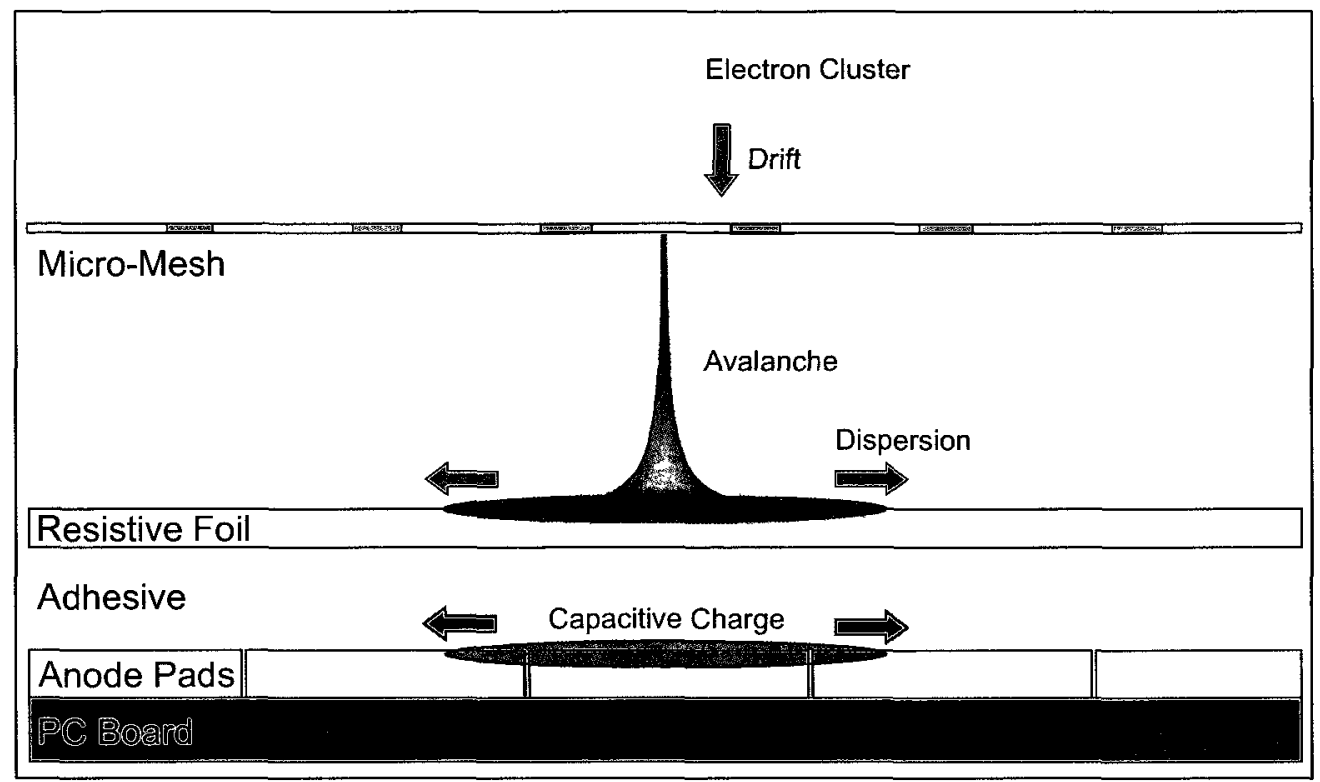

FIGURE 3.4: Sketch of an MPGD-TPC readout plane with charge dispersion. The resistive anode, dielectric adhesive layer, and readout pads, form a 2-dimensional distributed RC network.
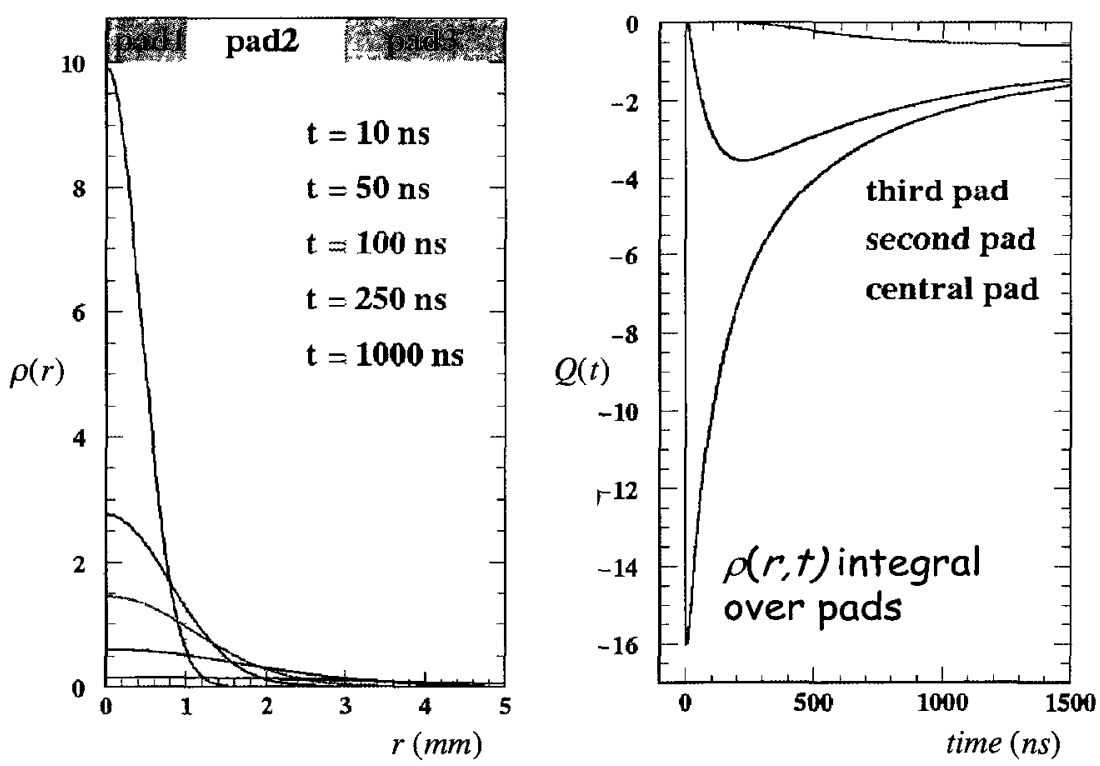

FIgURE 3.5: The charge density, $\rho(r)$, and integrated total charge, $Q(t)$ resulting from charge dispersion in a simulated resistive anode MPGD TPC [14]. 
goal of achieving high, uniform, resistivity.

\subsubsection{Readout}

Pulses are read out with 126 rectangular pads of $6 \times 2 \mathrm{~mm}^{2}$, which are contained in 7 rows of 18 pads each. There are also 2 outer rows, each having a single $36 \times 6 \mathrm{~mm}$ pad used as event vetoes. Each pad is connected to a charge-preamplifier from the ALEPH TPC, and a $200 \mathrm{MHz}, 8$-bit FADC. The pads integrate the time dependent charge density to give a resulting signal that represents the total charge on a pad as a function of time. The FADCs digitize the amplified charge signals directly without an intermediate shaper into $5 \mathrm{~ns}$ wide bins ${ }^{1}$. Pad dimensions were chosen to demonstrate that the ILD transverse resolution goal could be achieved with an MPGD-TPC using wide pads with a charge dispersion readout. The pad width, which is the dimension perpendicular to the projected path of a typical particle onto the readout plane (in this case, the $x$-direction) is the most important factor for determining the single hit momentum precision of the TPC. Normally it must be narrow enough so that the cluster signal is picked up by more than a single pad, which is necessary for calculating the charge centroid. With twice the width of pads that a GEM based MPGD requires to achieve the ILD transverse resolution goal (100 $\mu \mathrm{m}$ after $2 \mathrm{~m}$ of drift), the addition of charge dispersion could allow for half the readout channels - a much simpler detector.

\footnotetext{
${ }^{1}$ Much slower FADCs could have been used, as will be demonstrated in the analysis and results of this thesis.
} 


\begin{tabular}{|c|c|c|c|c|c|c|c|c|c|c|c|c|c|c|c|c|c|c|c|}
\hline \multirow{2}{*}{\multicolumn{2}{|c|}{$\begin{array}{cc}\text { Row } & y(\mathbf{m m}) \\
1 & 24\end{array}$}} & \\
\hline & & \multicolumn{18}{|c|}{18} \\
\hline 2 & 18 & 11 & 10 & 5 & 4 & 31 & 30 & 25 & 24 & 19 & 17 & 46 & 42 & 38 & 34 & 62 & 58 & 54 & 50 \\
\hline 3 & 12 & 14 & 9 & 8 & 3 & 2 & 29 & 28 & 23 & 22 & 48 & 45 & 41 & 37 & 33 & 61 & 57 & 53 & 49 \\
\hline 4 & 6 & 13 & 12 & 7 & 6 & 1 & 32 & 27 & 26 & 21 & 20 & 44 & 40 & 36 & 64 & 60 & 56 & 52 & 16 \\
\hline 5 & 0 & 79 & 115 & 119 & 123 & 127 & 99 & 103 & 107 & 111 & 47 & 43 & 39 & 35 & 63 & 59 & 55 & 51 & 15 \\
\hline 6 & -6 & 80 & 116 & 120 & 124 & 128 & 100 & 104 & 108 & 84 & 85 & 90 & 91 & 96 & 65 & 70 & 71 & 76 & 77 \\
\hline 7 & -12 & 113 & 117 & 121 & 125 & 97 & 101 & 105 & 109 & 112 & 86 & 87 & 92 & 93 & 66 & 67 & 72 & 73 & 78 \\
\hline 8 & -18 & 114 & 118 & 112 & 126 & 98 & 102 & 106 & 110 & 81 & 83 & 88 & 89 & 94 & 95 & 68 & 69 & 74 & 75 \\
\hline 9 & -24 & & & & & & & & & 82 & & & & & & & & & \\
\hline & $x(\mathrm{~mm})$ & -17 & -15 & -13 & -11 & -9 & -7 & -5 & -3 & -1 & 1 & 3 & 5 & 7 & 9 & 11 & 13 & 15 & 17 \\
\hline & Column & 1 & 2 & 3 & 4 & 5 & 6 & 7 & 8 & 9 & 10 & 11 & 12 & 13 & 14 & 15 & 16 & 17 & 18 \\
\hline
\end{tabular}

FIGURE 3.6: This figure shows the mapping of channel numbers on the readout plane of the COSMo TPC. Rows 1 and 9 long single pads that are used as event vetoes.

The pad height dimension, in the direction parallel to the projected path of a typical particle (y-direction) determines the track segmentation size, and therefore the number of space points used in track fitting. Since 200 track points are required by the ILD-TPC to meet the momentum resolution goal, and the effective TPC radius is planned to be $1280 \mathrm{~mm}$, the pads must be $\sim 6 \mathrm{~mm}$ in height. 


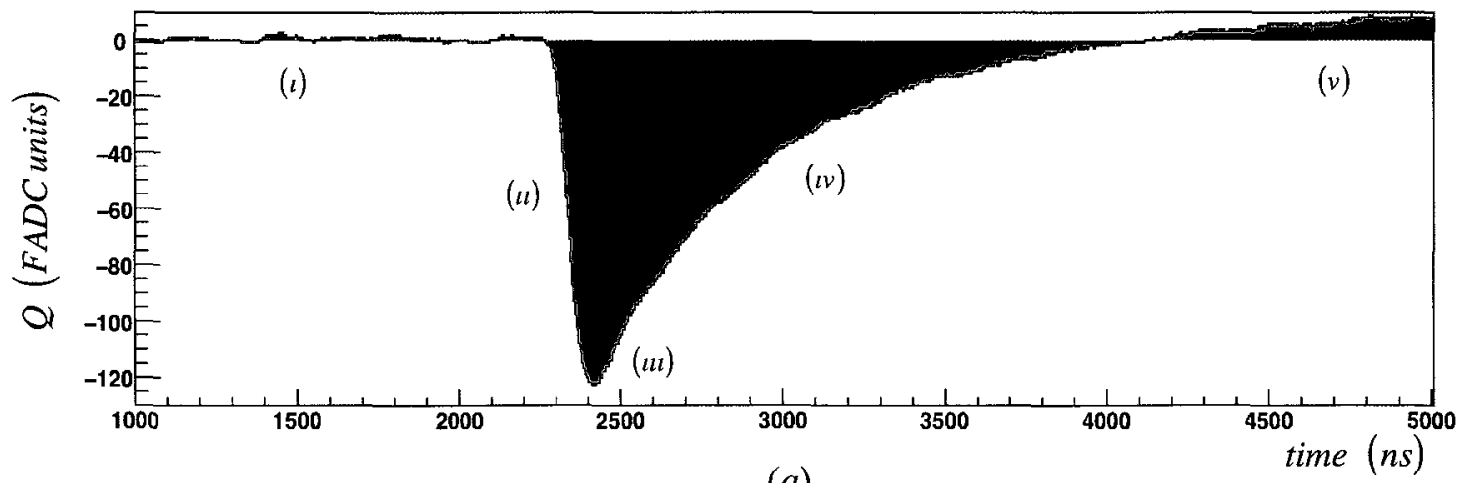

(a)

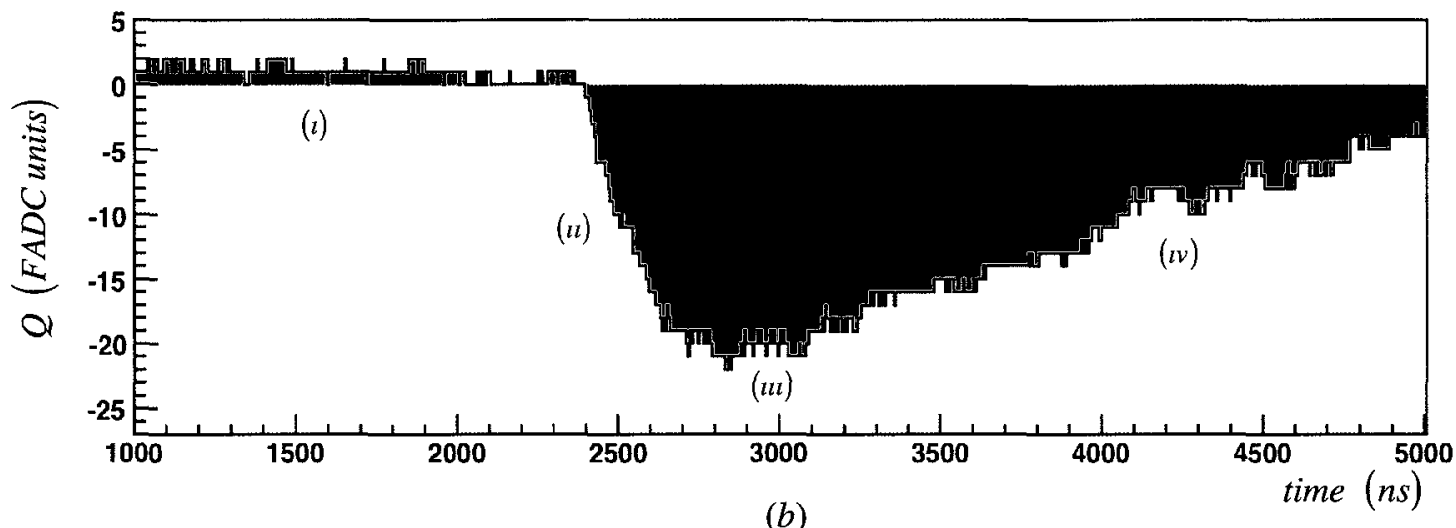

(b)

FIGURE 3.7: Typical pulses readout from the COSMo TPC. The pulse in (a) is due to direct charge collection, while (b) is a result of charge dispersion. The important characteristics are: (i) baseline noise, (ii) leading edge, (iii) charge collection peak, (iv) decay, and (v) over-shoot (an artifact produced by the electronics). Note the difference of vertical scale.

\subsubsection{Pulses}

The shape and amplitude of the charge pulse read off a particular pad depends on many different factors, including: the pad shape, RC time constant of the readout, where the projection of the track is with respect to the pad (x-position), drift distance, diffusion, and track angle. The pad geometry and RC time constant are optimized and fixed in our experiments, so we can't observe those particular effects. 

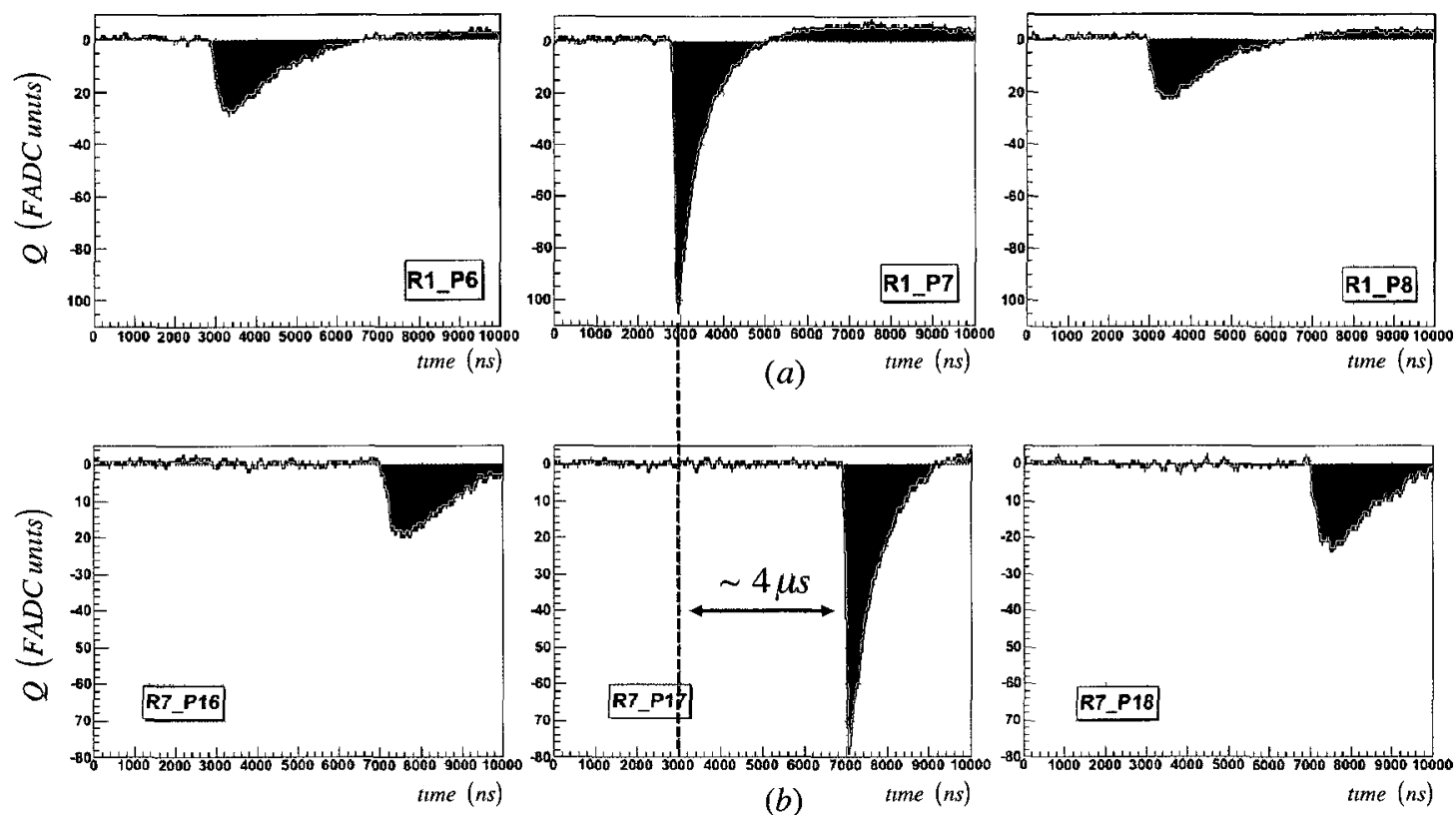

(a)
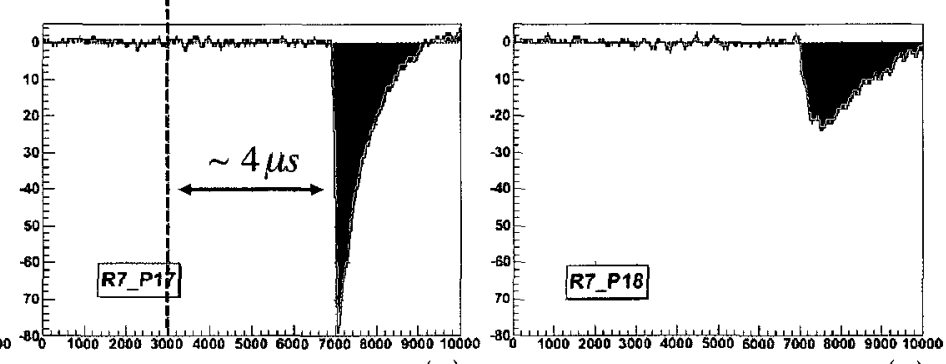

(b)

tame $(n s)$

tume $(n s)$

FIGURE 3.8: Pulses read out from two events in the COSMo TPC, showing the primary pulse in the center, and pulses from the two adjacent pads, the so-called nearest neighbours. Figure (a) is from a track with short drift distance (KEK, Run 45, Event 25, $z=12 \mathrm{~mm}$ ), and Figure (b) is taken from a track with a much longer distance (KEK, Run 45, Event 93, $z=137 \mathrm{~mm}$ ). The difference in drift times is approximately $4 \mu s$.

The location of the track projection with respect to the pad determines how the pulse is generated. For example, if the projection lies on the pad, then signal will be generated by direct charge, as shown in Figure $3.7 \mathrm{a}$, and is defined as the primary charge pulse on the row. The primary charge pulse is easily identified because it has the highest amplitude. For other pads in the row, the pulse is generated by charge dispersion across the resistive film (or a combination of direct charge and charge dispersion). The pulses directly adjacent to the primary charge pulse are referred to as the nearest nezghbour pulses, and have a lower signal-noise ratio and longer rise time, as shown in Figure 3.7b.

We can observe the effect of drift distance and track angle $(\theta)$ in the COSMo 
TPC from pulse characteristics. The drift time, which we define as the elapsed time between an event trigger and the mean arrival time of charge at the MPGD, causes a horizontal shift of the pulse (along the time axis) as well as a stretch in the leading edge of the pulse due to the additional time for longitudinal diffusion of the cluster. A particle passing through the TPC at an angle, $\theta$, with respect to the readout plane, will generate primary pulses with increasing (or decreasing) drift times between rows, and increasing (or decreasing) rise times. This effect can be seen in Figure 3.9 which displays the readout from all 126 pads from a single event, and Figure 3.10, which shows the primary pulses from each pad row for the same event. 


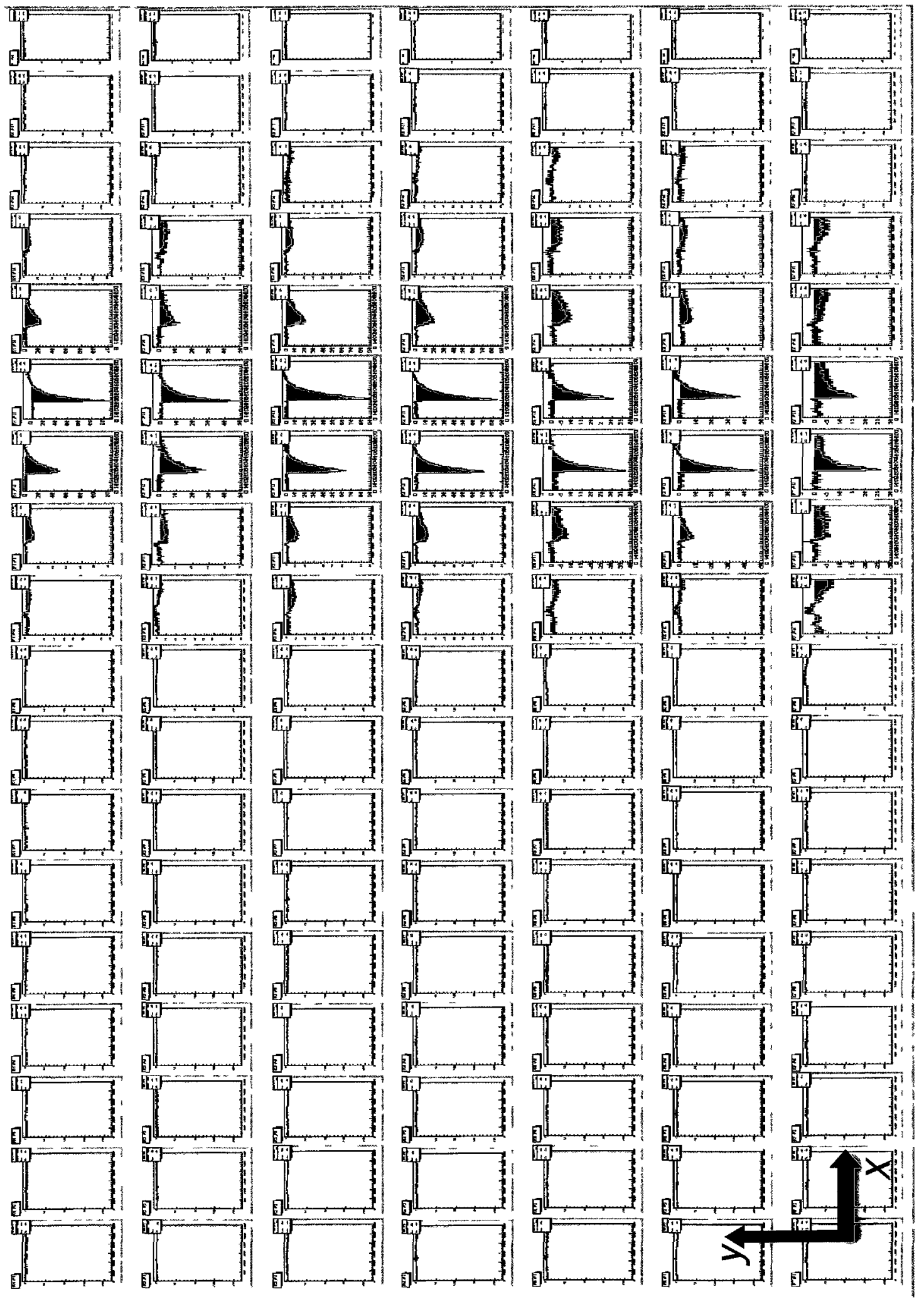

Figure 3.9: Pulses from all 126 pads (DESY, Run 6007, Event 243). 

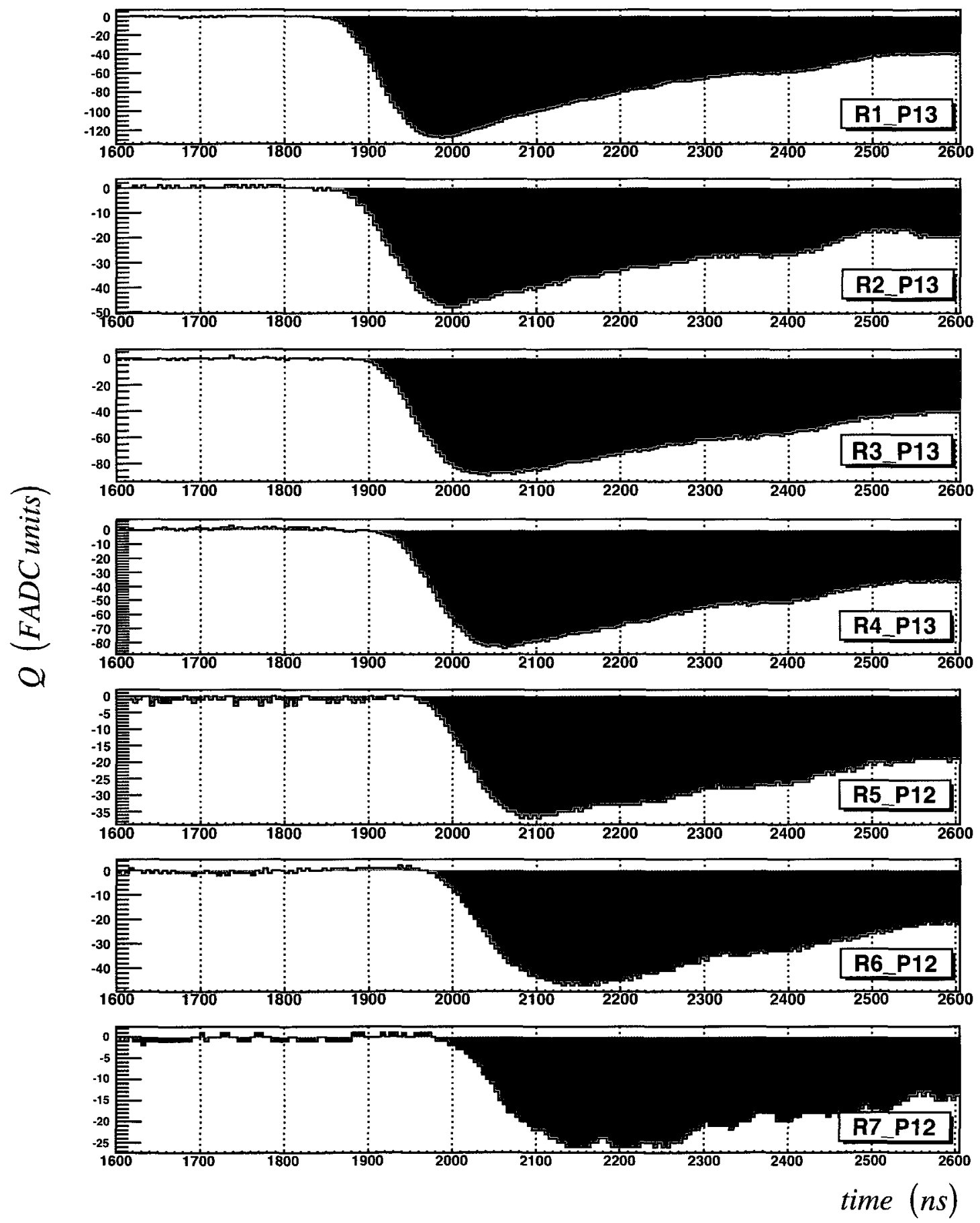

Figure 3.10: Primary pulses from each of the 7 readout rows. The increase in $t_{z e r o}$ (and rise-time) from Row 1 to Row 7 indicates a track angle $\theta$ with respect to the anode plane (DESY, Run 6007, Event 243). 


\section{Chapter 4}

\section{Experiments}

Data analyzed for the time resolution study in this thesis were previously collected from two different tests of a prototype MPGD TPC with charge dispersion. The first tests took place at KEK in the Fall of 2005, using a beam of high energy pions. The second test was conducted at DESY in Hamburg, using cosmic ray events in a high (5 T) magnetic field. The original analyses of both tests were focused on measuring the transverse resolution; the main results have been included at the end of this chapter.

\subsection{KEK Beam Test}

In the Fall of 2005, the first beam tests of the COSMo TPC (then known as the Carleton TPC) were conducted at KEK, a high energy research facility in 


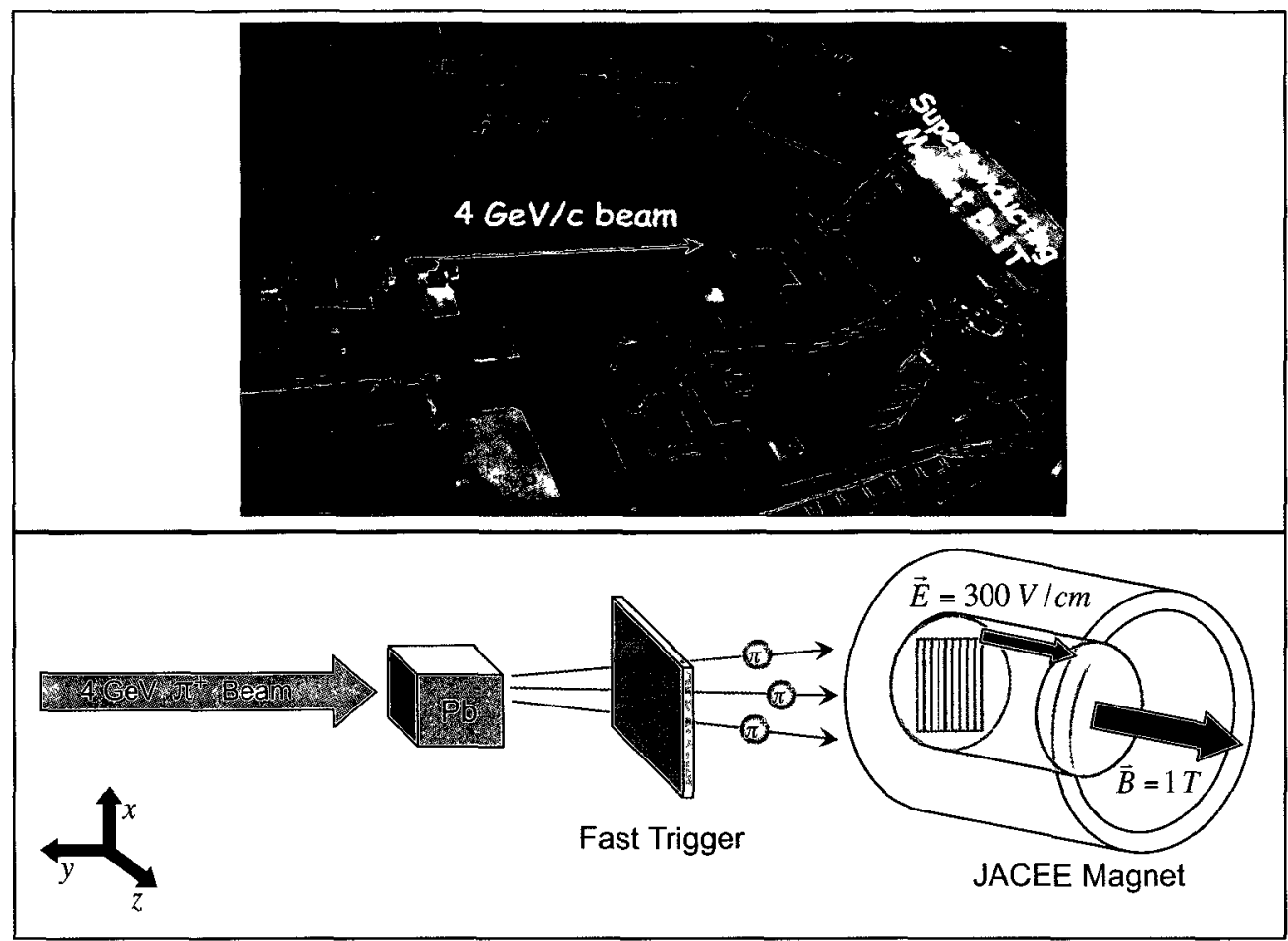

FIgURE 4.1: Photograph and sketch of the test of the COSMo TPC in a $4 \mathrm{GeV}$ $\pi^{+}$beam and $1 \mathrm{~T}$ superconducting magnet, at the KEK laboratory in Tsukuba, Japan.

Tsukuba, Japan. A $4 \mathrm{GeV} \pi^{+}$beam was directed at the TPC which was situated inside the $1 \mathrm{~T}$ super-conducting Jacee magnet, which has an inner diameter of $85 \mathrm{~cm}$ and an effective length of $1.5 \mathrm{~m}$. The ultra-thin solenoid was developed at KEK as a backup for the BESS-Polar instrument (Balloon-borne Experiment with Superconducting Spectrometer) [15]. A lead brick was located upstream in the beam path to disperse the beam, such that scattered particles would cover the full drift region of the TPC. This setup is shown in Figure 4.1. The chosen gas mixture was $95 \%$ argon $(\mathrm{Ar})$ and $5 \%$ isobutane $\left(\mathrm{C}_{4} \mathrm{H}_{10}\right)$, for a low transverse diffusion of $126 \mu \mathrm{m} / \sqrt{\mathrm{cm}}$ in the relatively weak $1 \mathrm{~T}$ magnetic field at $70 \mathrm{~V} / \mathrm{cm}$, $[6]$. 


\subsubsection{KEK Run Summary}

\begin{tabular}{ccccccr}
\hline \multirow{2}{*}{ Run } & \multirow{2}{*}{ Date } & \multirow{2}{*}{ Time } & \multicolumn{3}{c}{ Voltage Settings } & \multirow{2}{*}{ Events } \\
\cline { 4 - 6 } & & & $V_{\text {drift }}$ & $V_{\text {last }}$ & $V_{\text {mesh }}$ & \\
\hline 40 & $19 / 10 / 05$ & $9: 27-10: 10$ & 1450 & 400 & 351 & 3879 \\
41 & $19 / 10 / 05$ & $10: 10-11: 19$ & 1450 & 400 & 350 & 3879 \\
42 & $19 / 10 / 05$ & $11: 19-12: 12$ & 1450 & 400 & 350 & 3879 \\
43 & $19 / 10 / 05$ & $12: 12-12: 57$ & 1451 & 400 & 351 & 3879 \\
44 & $19 / 10 / 05$ & $12: 57-13: 46$ & 1452 & 400 & 351 & 3879 \\
45 & $19 / 10 / 05$ & $13: 46-14: 08$ & 1452 & 400 & 351 & 986 \\
\hline
\end{tabular}

TABLE 4.1: KEK experiment parameters and information on runs used in this thesis.

\subsection{DESY High Magnetic Field Test}

In the Fall of 2006, the COSMo TPC was tested at DESY in Hamburg, Germany, where the DESY superconducting magnetic provided fields up to $5 \mathrm{~T}$, as shown in Figure 4.2. Cosmic ray data were collected over a period of about four weeks. The gas mixture was changed to the so-called T2K gas, $95 \%$ argon, $3 \%$ carbon-tetrafluoride (CF4), and $2 \%$ isobutane, which is being used in the T2KNear Detector and is considered a possible candidate for the ILD TPC. It has a high $73 \mu \mathrm{m} / \mathrm{ns}$ electron drift velocity at a moderate $200 \mathrm{~V} / \mathrm{cm}$ electric field, and a large $\omega \tau \sim 20$ at $5 \mathrm{~T}$, which reduces transverse diffusion to $\mathrm{D}_{\mathrm{T}} \simeq 19 \mu \mathrm{m} / \sqrt{\mathrm{cm}}$. The transverse TPC resolution was measured at $5 \mathrm{~T}$ to benchmark TPC performance in a magnetic field of strength comparable to that of the ILC detector. 

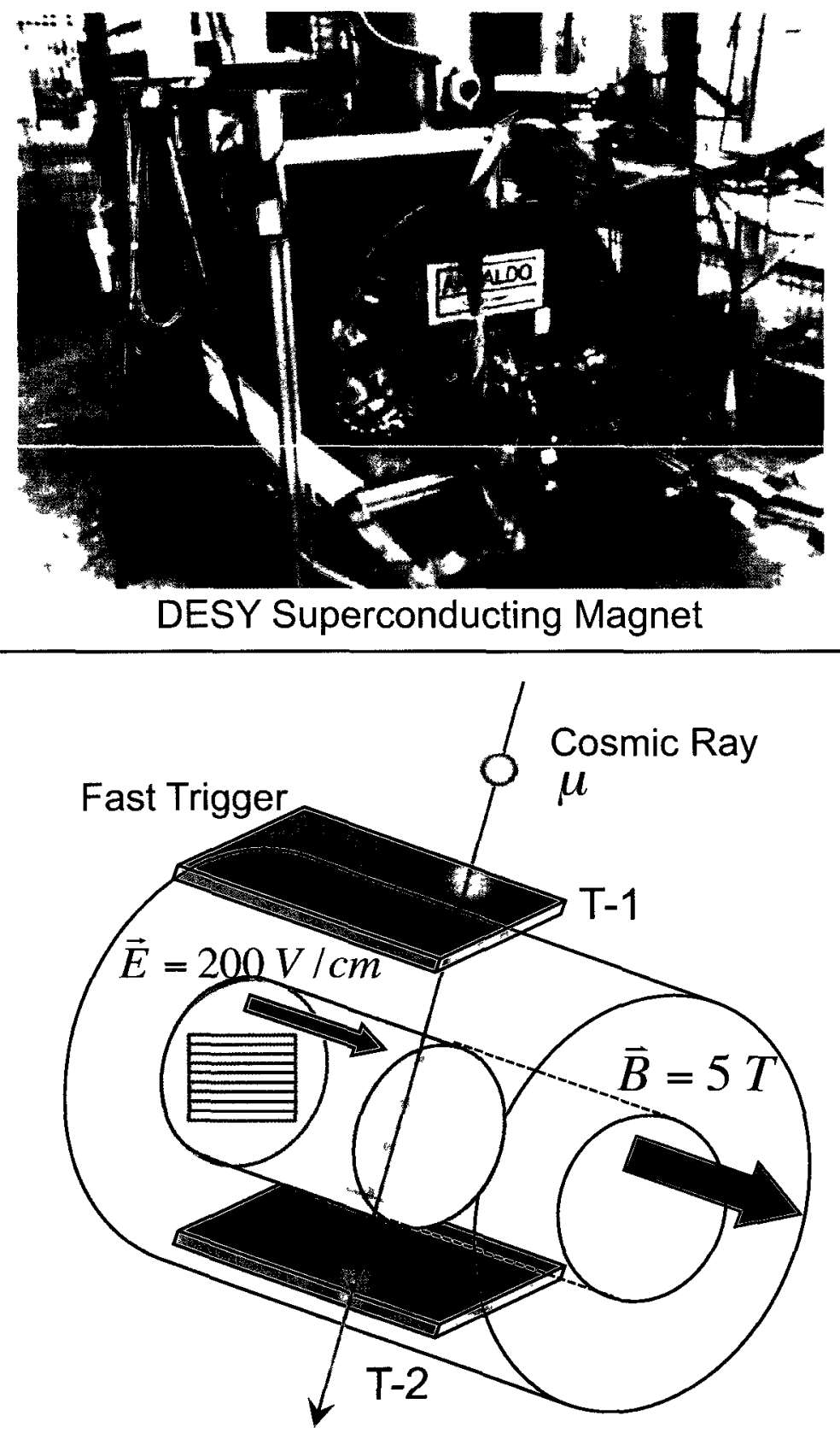

FIgure 4.2: Photograph and diagram of the cosmic ray test of the COSMo TPC in $5 \mathrm{~T}$ superconducting magnet, at the DESY laboratory in Hamburg, Germany. 


\subsubsection{DESY Run Summary}

\begin{tabular}{ccccccccc}
\hline \multirow{2}{*}{ Run } & \multirow{2}{*}{$\begin{array}{c}\text { Start } \\
\text { Date }\end{array}$} & Elapsed & \multicolumn{2}{c}{ Voltage Settings } & Cosmic & Filtered & Events \\
\cline { 5 - 6 } & Time & $V_{\text {drift }}$ & $V_{\text {last }}$ & $V_{\text {mesh }}$ & Rate & Rate & \\
\hline 6007 & $25 / 11 / 06$ & $17 \mathrm{~h} 7$ & 3470 & 470 & 330 & $51 /$ min & $2.0 /$ min & 2012 \\
6008 & $26 / 11 / 06$ & $50 \mathrm{~h} 20$ & 3465 & 470 & 330 & $51 /$ min & $2.0 /$ min & 6048 \\
6009 & $28 / 11 / 06$ & $47 \mathrm{~h} 43$ & 3401 & 470 & 330 & $50 /$ min & $2.0 /$ min & 5681 \\
6010 & $30 / 11 / 06$ & $45 \mathrm{~h} 9$ & 3472 & 470 & 330 & $50 /$ min & $2.0 /$ min & 5288 \\
6011 & $2 / 12 / 06$ & $52 \mathrm{~h} 5$ & 3472 & 470 & 330 & $50 /$ min & $2.0 /$ min & 6333 \\
\hline
\end{tabular}

TABLE 4.2: DESY experiment parameters and information on runs used in this thesis.

\subsection{Previous Analysis Results}

Results for the analysis of the transverse resolution from the $2005 \mathrm{KEK}$ experiment are shown in Figure 4.3, and Figure 4.4 from [16]. The resolution degrades with drift distance, however this is due to the $126 \mu \mathrm{m} / \sqrt{\mathrm{cm}}$ transverse diffusion. An extrapolation to zero drift distance yields $\epsilon_{0}=50 \mu \mathrm{m}$. A stronger field would flatten the resolution curve to $\sim \epsilon_{0}$, so this was an excellent result.

Figures 4.5 and 4.6 shows an analysis of the 2006 DESY experiment from [17]. The smaller $19 \mu \mathrm{m} / \sqrt{\mathrm{cm}}$ transverse diffusion, due to the $5 \mathrm{~T}$ field and $\mathrm{T} 2 \mathrm{~K}$ gas, is clearly reflected in the near constant resolution over the full drift length of the TPC. These results are the best ever produced by a TPC with $2 \mathrm{~mm}$ wide pads, and show that the ILD-TPC target of $100 \mu \mathrm{m}$ after $2 \mathrm{~m}$ of drift is possible ${ }^{1}$.

\footnotetext{
${ }^{1}$ An improved analysis technique has reduced $\epsilon_{0}$ further to $\sim 30 \mu \mathrm{m}$, however this result has not yet been unpublished.
} 

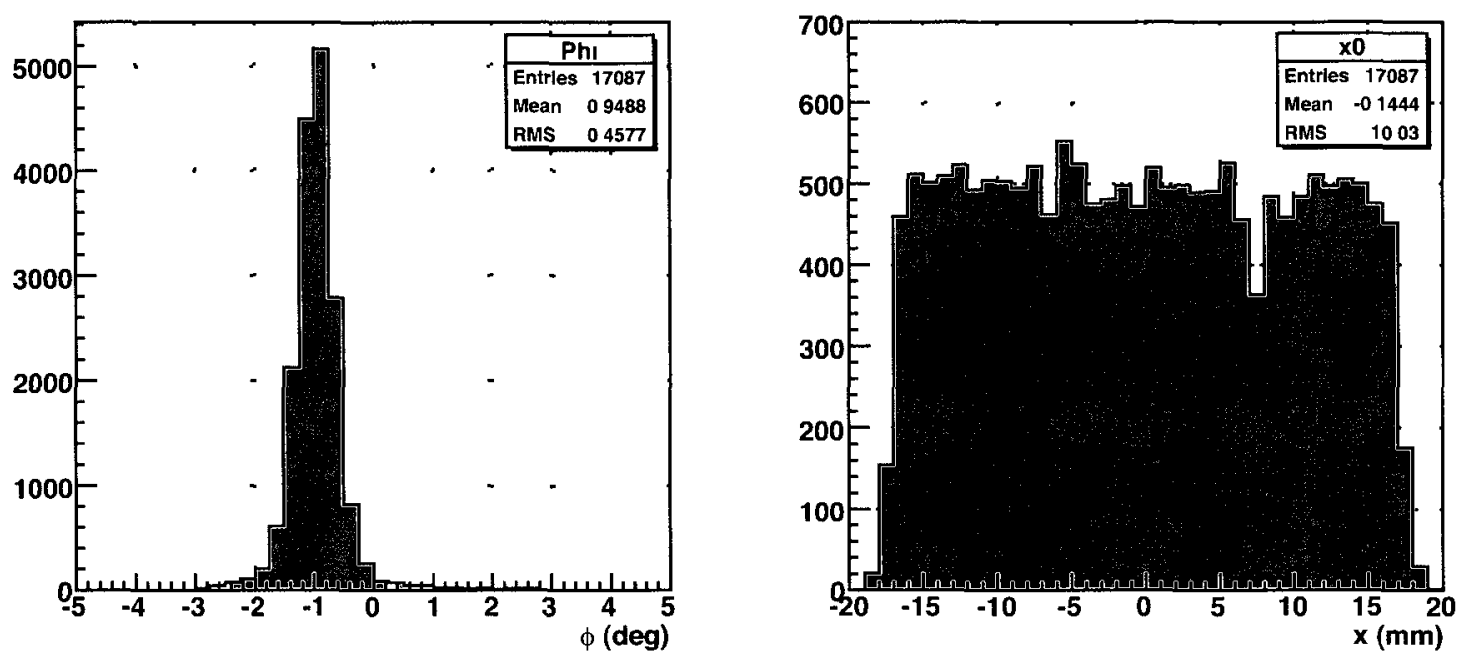

Figure 43 Distributions of $\phi$ and $x$ (center row) of pions in the KEK analysis The narrow width of the $\phi$ distribution reflects the distance between the lead brick (used to scatter the beam) and the TPC

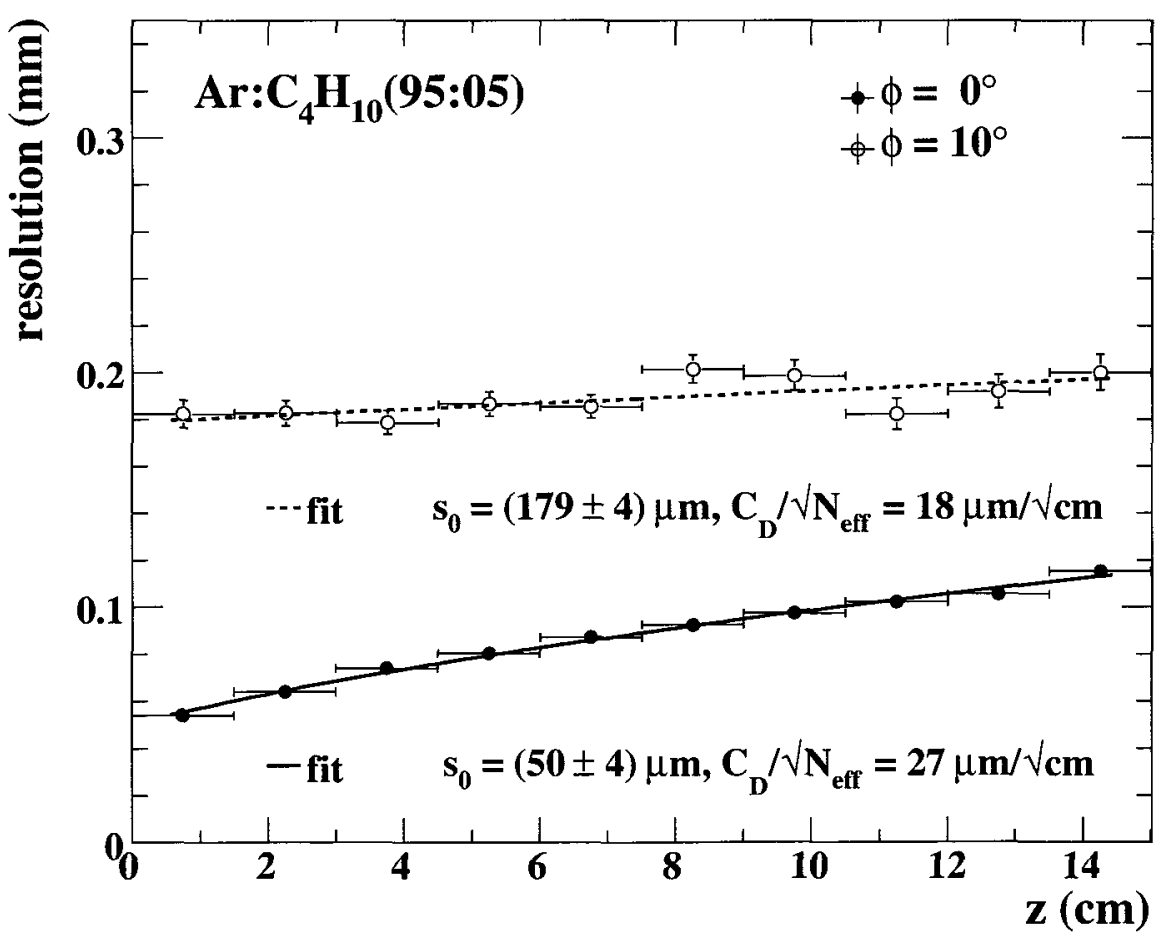

Figure 44 Transverse resolution of the COSMo TPC for $4 \mathrm{GeV} \pi^{+}$particles in a $1 \mathrm{~T}$ magnetic field The upper curve shows the effect of track angle $\phi$ on the resolution [16] 

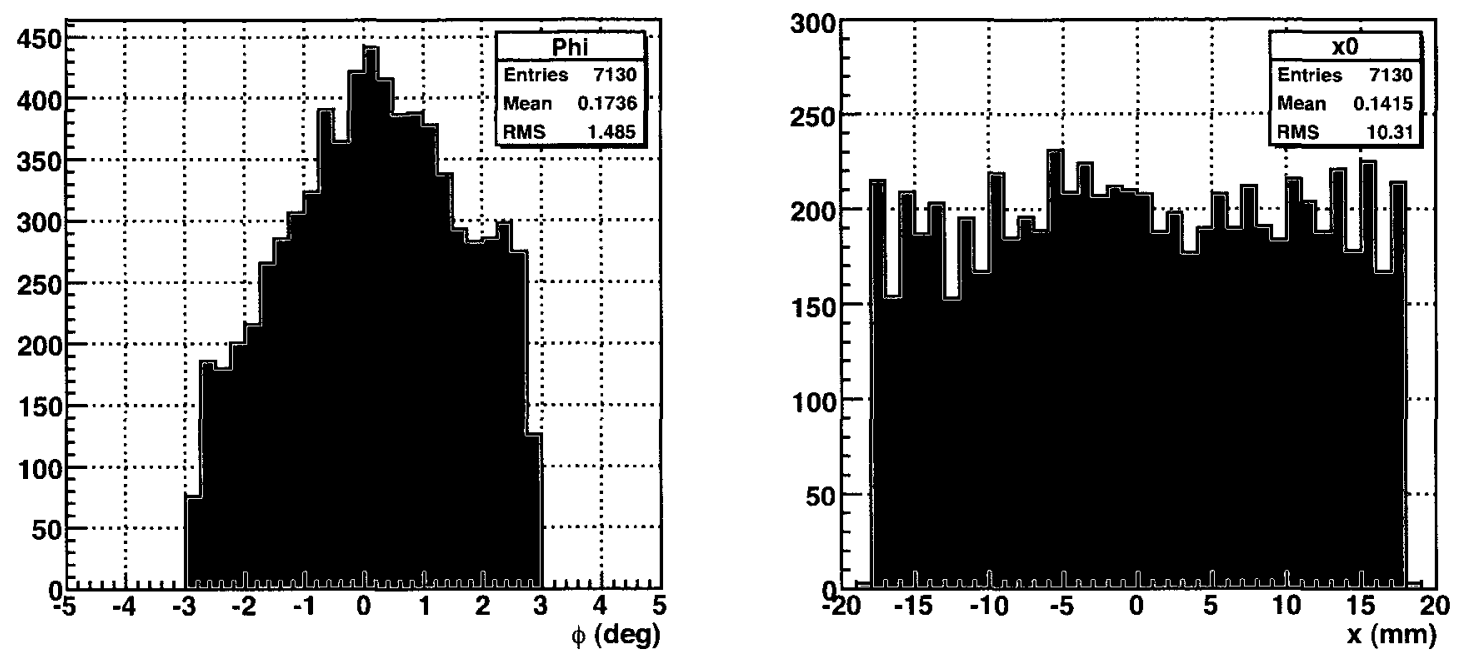

FIGURE 4.5: Distributions of $\phi$ and $x$ (center row) of cosmic rays in the DESY analysis.

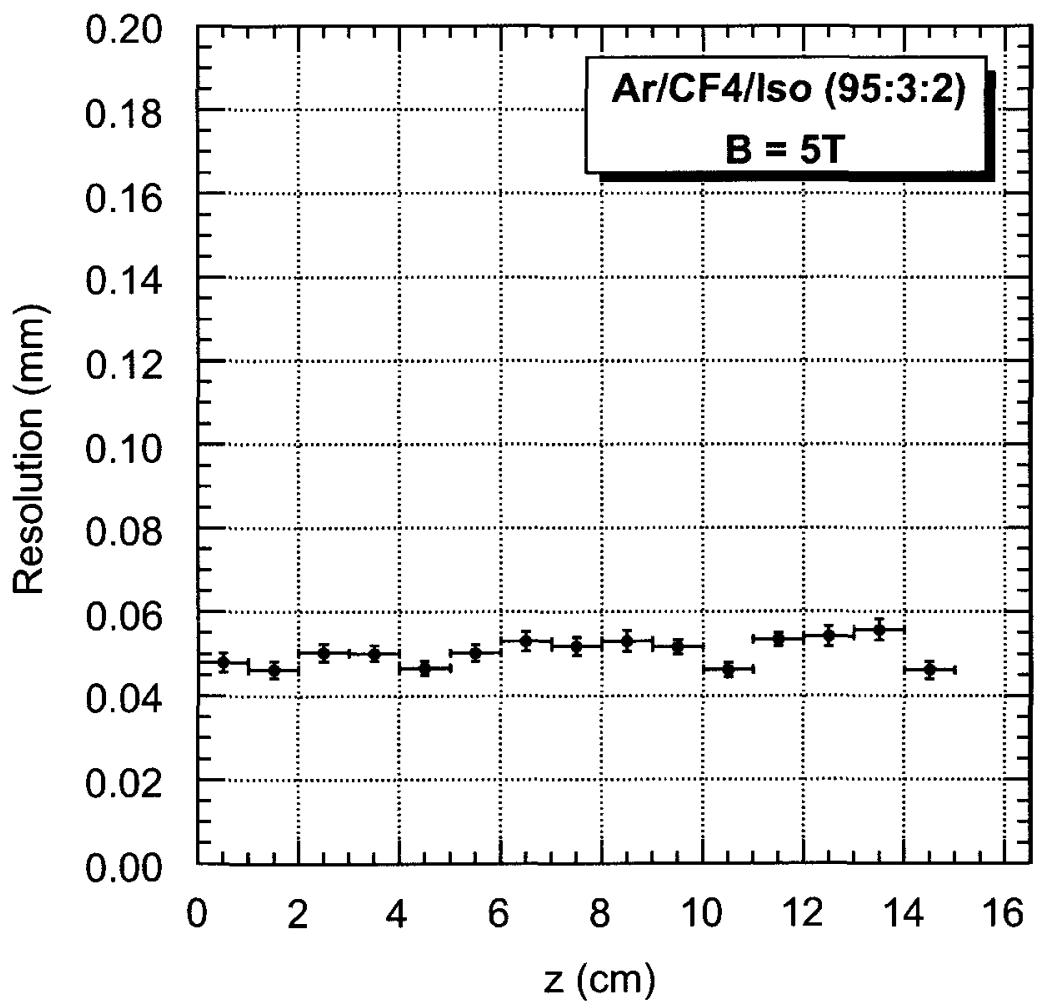

FIgURE 4.6: Transverse resolution of COSMo TPC for cosmic rays in a $5 \mathrm{~T}$ magnetic field [17]. 


\section{Chapter 5}

\section{Time Analysis}

Successful track fits in the readout plane $(x, y)$, previously done for the transverse resolution analysis, are used to select tracks for an analysis of the time resolution. Data for these tracks were re-analyzed by a program written in the ROOT [18] framework which determines their timing, leading to a linear fit of the track projected into the $(z(t), y)$ plane. This allows for approximate measurements of drift velocities and the longitudinal diffusion constants as consistency tests, and ultimately the single hit longitudinal resolution.

\subsection{Pulse Timing}

To perform a track fit in $(z(t), y)$, as well as measure properties such as drift velocity, diffusion, and resolution, we need a consistent method of determining 
the time of the primary charge signals for each row of pads in the readout. To accomplish this, it is necessary to identify a characteristic feature of the pulse. One choice would be to choose the time that the pulse starts to rise above baseline noise, which would correspond to the time that charge begins to arrive at the pad row (depicted as $t_{\text {begin }}$ in Figure 5.1). Although this may be a good choice for some detectors such as photomultipliers, it would be a poor choice for a TPC. As discussed in Chapter 2, drift clusters have a Gaussian profile due to diffusion, and therefore the first charges that arrive at the anode would be from the leading tanl of the longitudinal diffusion Gaussian where the density is low, which would carry a large uncertainty. This is depicted in Figure 5.1. This same argument also applies to the choice of the pulse peak ( $t_{\text {end }}$ in Figure 5.1) as it is also located in the Gaussian tail.

For a drift chamber, the correct choice of arrival time $\left(t_{\mathrm{zero}}\right)$ is the mean arrival time of the cluster ( $t_{\text {mean }}$ in Figure 5.1), which is the center of the longitudinal diffusion Gaussian. This corresponds to the inflection point on the leading edge of a primary charge pulse - the time when roughly half the total charge has been collected. So determining the arrival time is reduced to finding an algorithm which consistently identifies the inflection point of a primary charge pulse. 


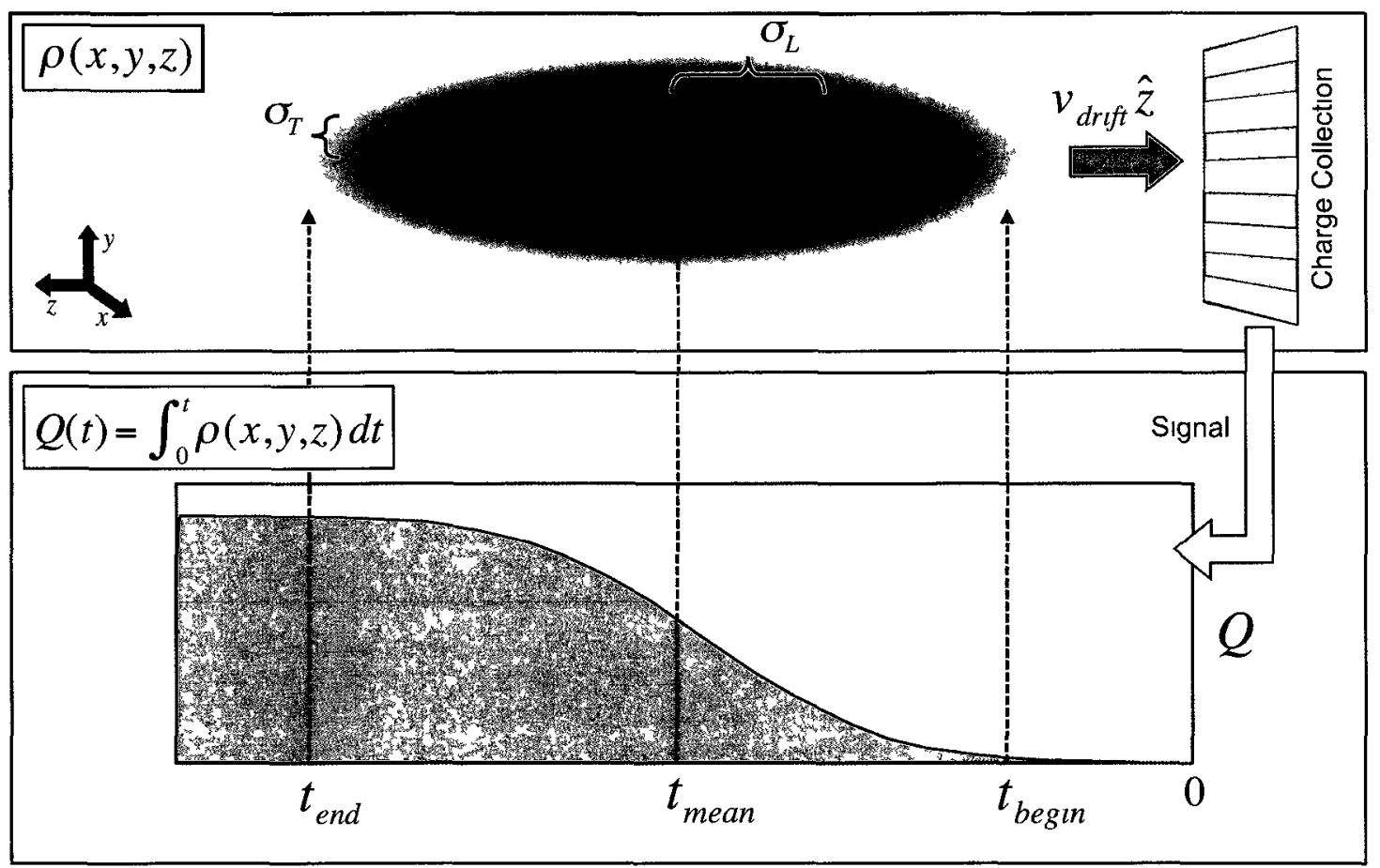

FIgURE 5.1: Sketch of the Gaussian density of a drift cluster arising from diffusion. The proper location to measure arrival time is $t_{\text {mean }}$, the center of the cluster.

\subsubsection{Differentiation Method}

Our first attempt at determining the mean cluster arrival time was to numerically differentiate the measured charge pulse, $Q(t)$, for the primary charge collecting pad. Because the readout pad effectively integrates a Gaussian density to obtain $\mathrm{Q}(\mathrm{t})$, the differentiated pulse, $I(t)$, contains a Gaussian that peaks at the time of maximum current in the Micromegas. This peak corresponds to the center of the charge distribution Gaussian, which is the mean arrival time.

Two different numerical methods were used to differentiate measured primary pulses: the so-called 3-point and 5-point formulas. The 3-point formula determines the derivative at a point by calculating the slope of the secant line 

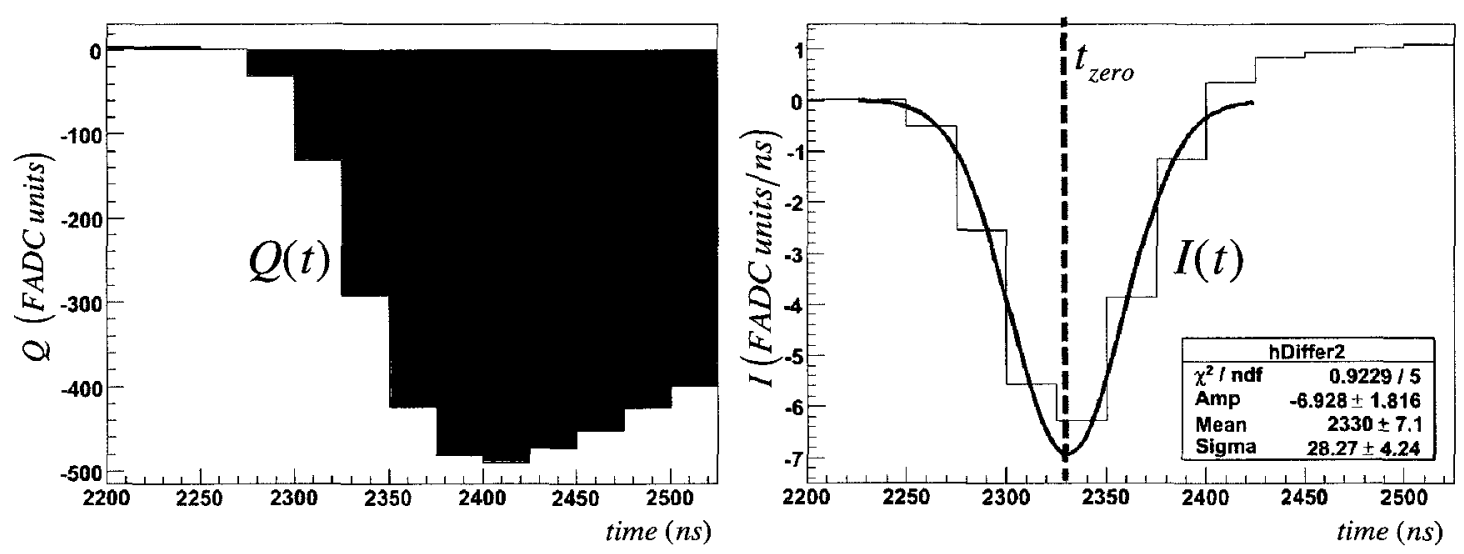

FIgURE 5.2: The primary charge pulse, $\mathrm{Q}(\mathrm{t})$, and the numerically differentiated pulse, $\mathrm{I}(\mathrm{t})$, using the 5 -point formula. The Gaussian fit determines the mean arrival time, $t_{\text {zero }}$.

drawn between the two adjacent points as follows:

$$
Q^{\prime}(t)_{3 p}=\frac{Q(t-h)-Q(t+h)}{2 h}
$$

where $h$ is the bin size. The 3 -point formula is accurate to $2^{\text {nd }}$ order. The 5 -point formula incorporates the next 2 nearest neighbouring points into the fit:

$$
Q^{\prime}(t)_{5 p}=\frac{Q(t-2 h)-8 Q(t-h)+8 Q(t+h)-Q(t+2 h)}{12 h},
$$

and is accurate to $4^{\text {th }}$ order. The derivation of these two approximations is explained in Appendix C. This method produces good results (using both algorithms), however, there were some drawbacks. The technique is sensitive to highfrequency noise, which becomes a problem when the signal-to-noise ratio is low. For such pulses, the differentiated noise peaks can be large enough to mask the true current peak. Such a situation is demonstrated in Figure 5.3, for two primary 

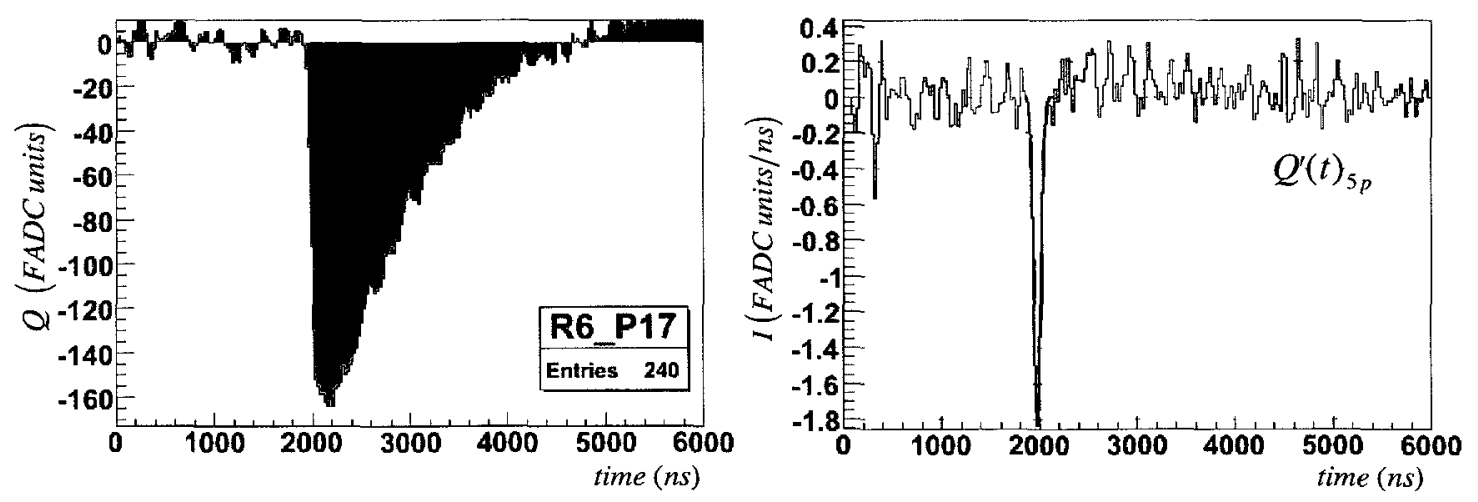

(a) High Amplitude Pulse
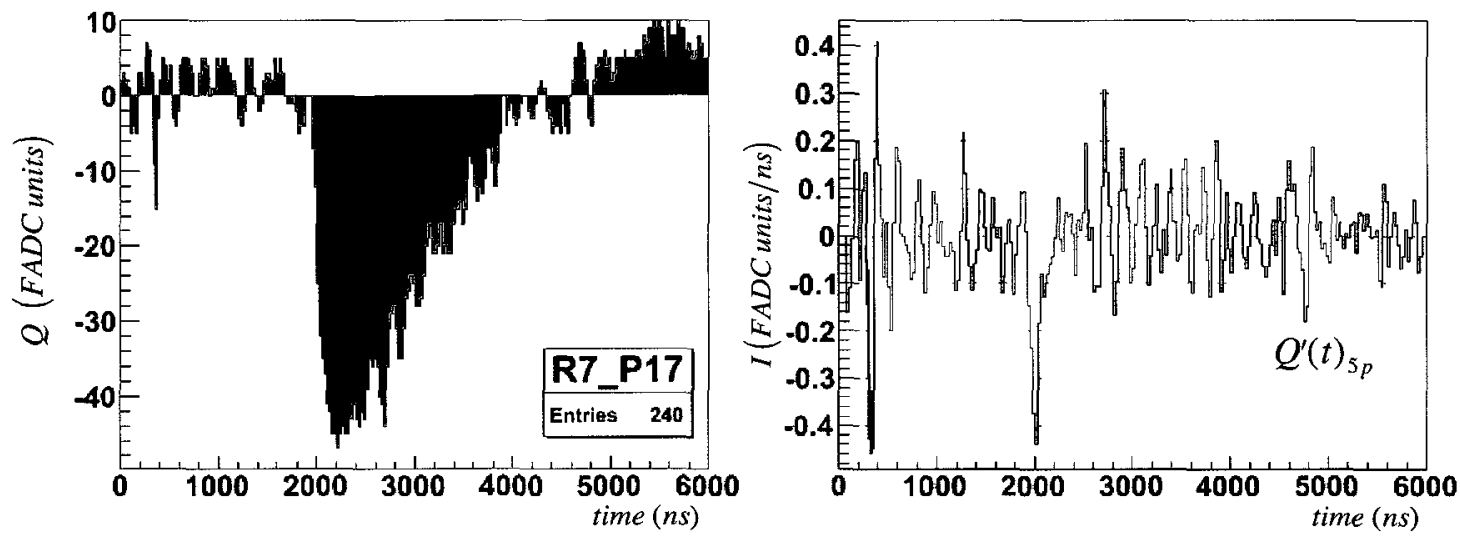

(b) Low Amplitude Pulse

Figure 5.3: Two primary pulses from neighbouring pad rows with similar high-frequency noise, but different amplitudes. In this example, the fit for $t_{z e r o}$ failed for the low amplitude pulse. (DESY, Run:6007, Event:1349)

charge pulses from neighbouring rows (6 and 7). The pulse from Row 6 has a large enough amplitude that the differentiated signal peak is easily identifiable from the noise, which is not the case for Row 7. To improve the probability of fitting the correct peak, it is necessary to scan the differentiated pulse to provide adequate estimates of the fit parameters, and this slows the algorithm considerably.

Problems caused by high-frequency noise can be reduced by using higher order differentiation formulas, which have the effect of smoothing out the noise, but the limited number of bins in the leading edge makes this undesirable. The 
problem could also be addressed using a discrete Fourier transform to calculate the low frequency components of the pulse. However, a new method was developed, that involves directly fitting an Error function to the pulse leading edge. This is described in the next section.

\subsubsection{Error Function Fit Method}

Again, we use the fact that the distribution of drifting electrons arriving at the micromegas is primarily determined by diffusion, and the longitudinal profile should follow a Gaussian. Thus, the collection of charge should behave as the running integral of a Gaussian, known as an Error function. Therefore the leading edge of the primary charge pulse, which has negative slope, is fit with a function of the form:

$$
Q(t) \approx \operatorname{Erf}(t)=\frac{A}{\sigma_{\mathrm{L}} \sqrt{2 \pi}} \int_{0}^{t} \exp \left[-\frac{1}{2}\left(\frac{t-t_{\text {zero }}}{\sigma_{\mathrm{L}}}\right)^{2}\right] d t .
$$

The inflection point is obtained directly from the fit parameter $t_{\text {zero }}$, which corresponds to the mean arrival time of the charge cluster at the primary pad. The parameter $\sigma_{L}$ corresponds to the characteristic rise-time of the pulse (which scales approximately with the charge cluster width), and parameter $A$ scales the amplitude. An additional parameter, $B$, is added to the error function to allow for a shift in the baseline, should this occur after the calculated baseline was subtracted. The fitting is procedure is carried out using the chi-squared minimization principle, which is explained in Appendix D. 


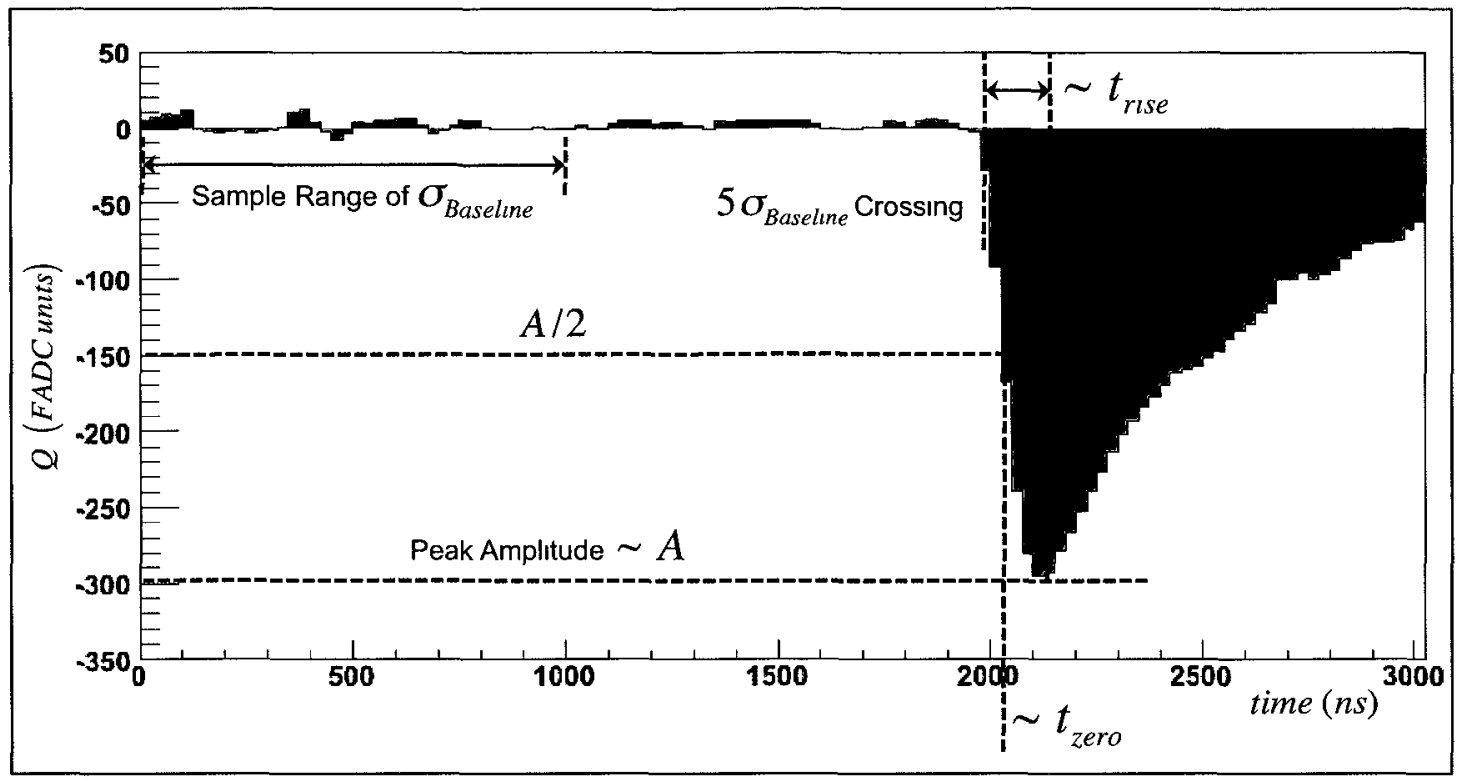

FIgURE 5.4: Parameter estimates of the Error function, which are required before the fitting routine can be called.

Before a charge pulse can be successfully fit, the algorithm requires a few critical inputs: the range of bins to include in the fit, and estimates of the fit parameters to use as a starting point in the chi-squared minimization procedure. First, the standard deviation of the baseline fluctuation $\left(\sigma_{\text {Baselne }}\right)$ is calculated using $1 \mu$ s of channels before the signal starts to rise, as shown in Figure 5.4. The time between the first bin with amplitude that exceeds the baseline by $5 \sigma_{\text {Baselne }}$, and the bin with the highest amplitude (peak time), gives an estimate of the pulse rise-time $\left(t_{\mathrm{rise}}\right)$. The fit range is made proportional to the rise-time. The start of the range is set to the time equal to $t_{\text {rise }} n$ s prior to the 5 -sigma crossing. The end of the fit range was originally set to peak time, however, we found that the fit results could be greatly improved by modifying a few consecutive bins after the channel with peak amplitude to also have the same amplitude. Depending on 


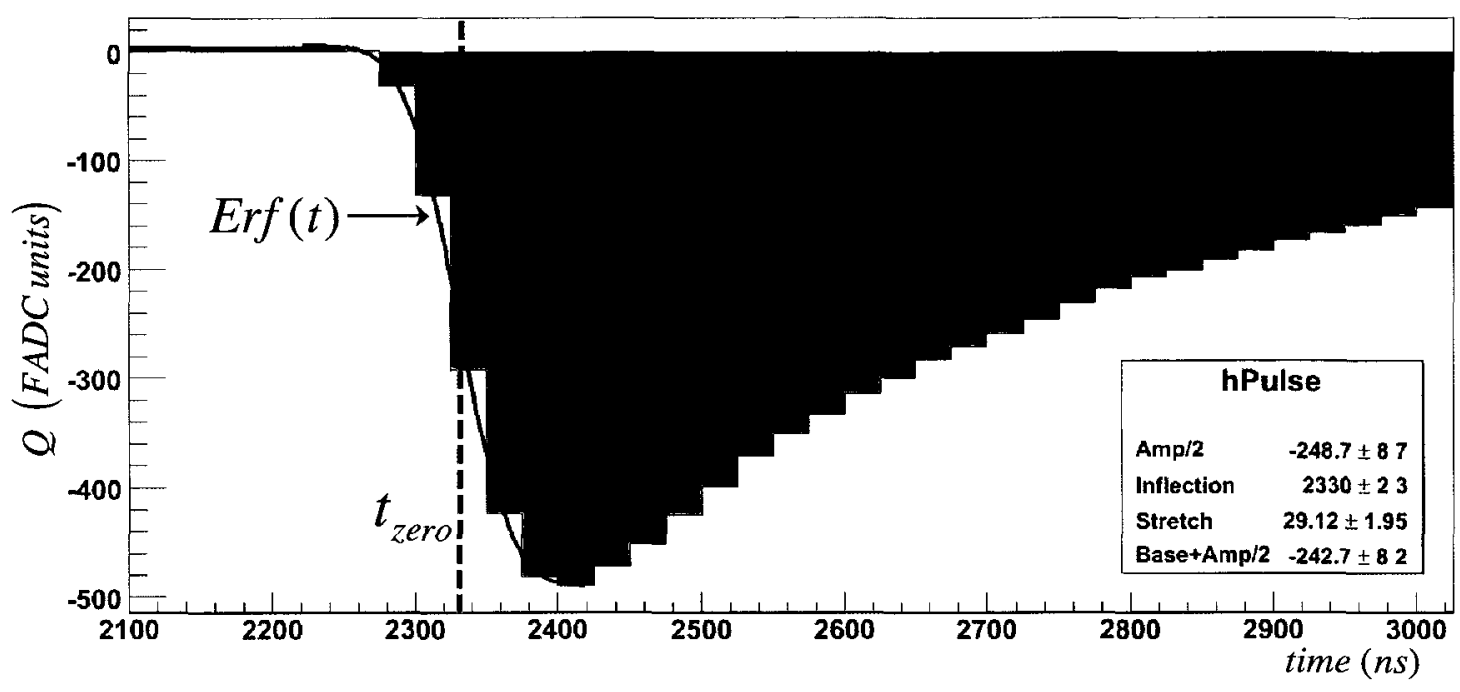

Figure 5.5: The Error function fit to the leading edge of the charge pulse. The inflection point, $t_{z e r o}$, is taken as the mean arrival time of charge at the pad.

the chosen bin-width for analysis, up to 10 bins were modified $^{1}$. Since the error function is symmetric about its inflection point, this modification greatly improved the probability of a successful fit.

The fit parameters were estimated as follows: (1) $t_{\text {zero }}$ is estimated by locating the first bin with an amplitude greater than half that of the peak amplitude, (2) $\sigma_{L}$ is estimated as $t_{\text {rise }}$ divided by 4 , and (3) $A, B$ are both estimated to be the highest amplitude of the pulse.

To compare the Error function fit of the raw pulse, shown in Figure 5.5, to the Gaussian fit of the differentiated pulse, the Error function fit parameters are extracted and substituted into a Gaussian function, which is plotted over top of the fitted differentiated pulse. The result is shown in Figure 5.6, which demonstrates that the two methods produce essentially the same results as expected.

\footnotetext{
${ }^{1}$ Attempts were made at avoiding this by convolving the error function with a decaying exponential function, but the probability of a successful fit was very low.
} 


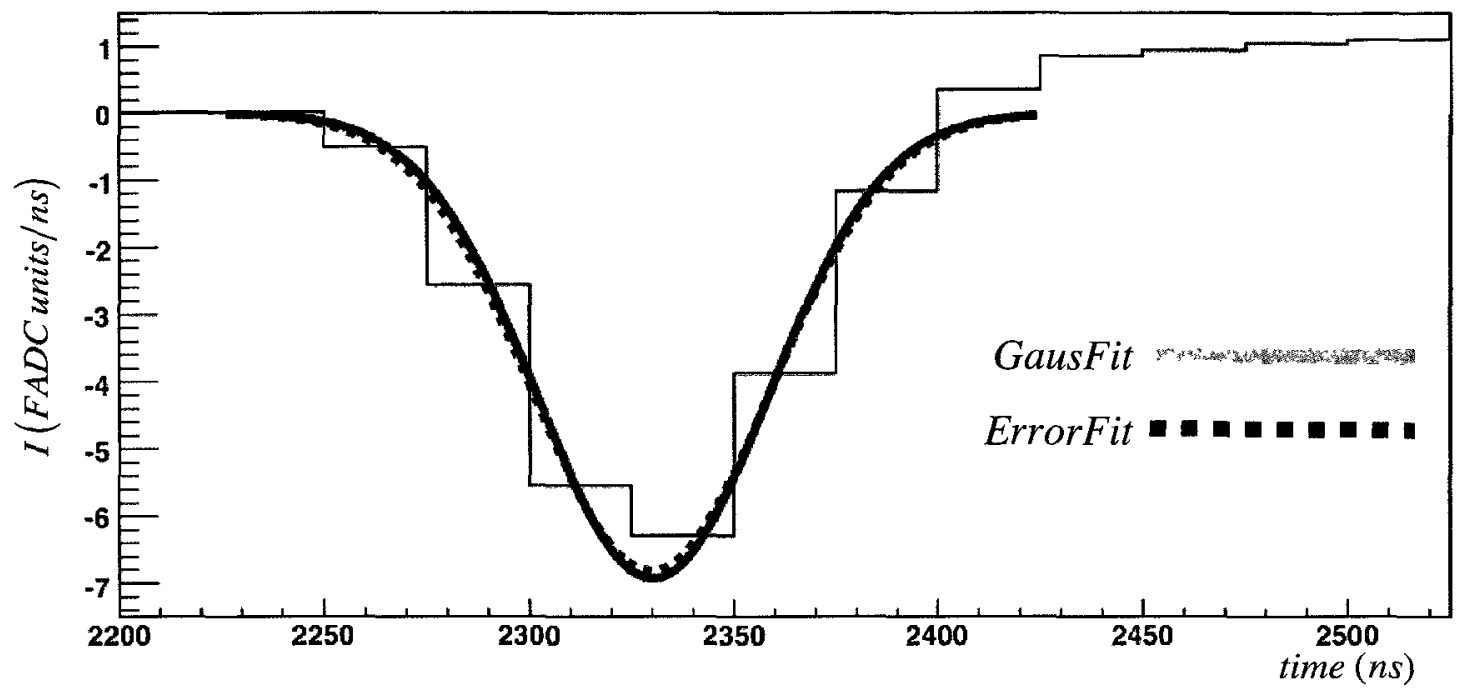

Figure 5.6: Comparison of results from an Error function fit of the raw pulse, with a Gaussian fit of the numerically differentiated pulse.

\subsection{Track Fit}

With the time for each primary charge pulse determined $\left(t_{\text {zero }}\right)$, and the corresponding pad centre known ( $y$ coordinate), the linear $(\mathrm{t}, \mathrm{y})$ track fit can be performed to determine the best estimate of the true track projection in this plane. ${ }^{2}$. The chi-squared minimization principle is used to derive the weighted least-squares equations for the parameters $\mathrm{A}$ and $\mathrm{B}$ in the straight line equation:

$$
t=A+B y .
$$

\footnotetext{
${ }^{2}$ See Appendix A for a justification of using a linear track fit in the $(y, t)$ plane.
} 
Since the $y$ coordinate is known (row position), and the $t$ coordinates have a statistical error, $\sigma_{t}$, from the pulse fit, we obtain ${ }^{3}$ :

$$
\begin{gathered}
A=\frac{\sum_{\imath=1}^{N} w_{\imath} y_{\imath}^{2} \sum_{\imath=1}^{N} w_{\imath} t_{\imath}-\sum_{\imath=1}^{N} w_{\imath} y_{\imath} \sum_{\imath=1}^{N} w_{\imath} y_{\imath} t_{\imath}}{\sum_{\imath=1}^{N} w_{\imath} \sum_{\imath=1}^{N} w_{\imath} y_{\imath}^{2}-\left(\sum_{\imath=1}^{N} w_{\imath} y_{\imath}\right)^{2}} \\
B=\frac{\sum_{\imath=1}^{N} w_{\imath} \sum_{\imath=1}^{N} w_{\imath} y_{\imath} t_{\imath}-\sum_{\imath=1}^{N} w_{\imath} y_{\imath} \sum_{\imath=1}^{N} w_{\imath} t_{\imath}}{\sum_{\imath=1}^{N} w_{\imath} \sum_{\imath=1}^{N} w_{\imath} y_{\imath}^{2}-\left(\sum_{\imath=1}^{N} w_{\imath} y_{\imath}\right)^{2}}
\end{gathered}
$$

where $A$ is the $t$-intercept and $B$ is the slope $\left(\frac{\Delta t}{\Delta y}\right), y_{\imath}$ is the row number, and $w_{\imath}$ is the weighting factor $\left(=1 / \sigma_{t_{2}}^{2}\right)$. The uncertainties in $\mathrm{A}$ and $\mathrm{B}$ are given by:

$$
\begin{aligned}
& \sigma_{A}^{2}=\frac{\sum_{\imath=1}^{N} w_{\imath} y_{\imath}^{2}}{\sum_{\imath=1}^{N} w_{\imath} \sum_{\imath=1}^{N} w_{\imath} y_{\imath}^{2}-\left(\sum_{\imath=1}^{N} w_{\imath} y_{\imath}\right)^{2}}, \\
& \sigma_{B}^{2}=\frac{\sum_{\imath=1}^{N} w_{\imath}}{\sum_{\imath=1}^{N} w_{\imath} \sum_{\imath=1}^{N} w_{\imath} y_{\imath}^{2}-\left(\sum_{\imath=1}^{N} w_{\imath} y_{\imath}\right)^{2}} .
\end{aligned}
$$

An example of the weighted least-squares fit to the reconstructed points from a single event is shown in Figure 5.7. The error bars, extracted from the charge pulse fit for $t_{\text {zero }}$, determine the weights assigned to each point in the fit.

\footnotetext{
${ }^{3}$ See Appendix D for the derivation of the weighted least-squares equations.
} 


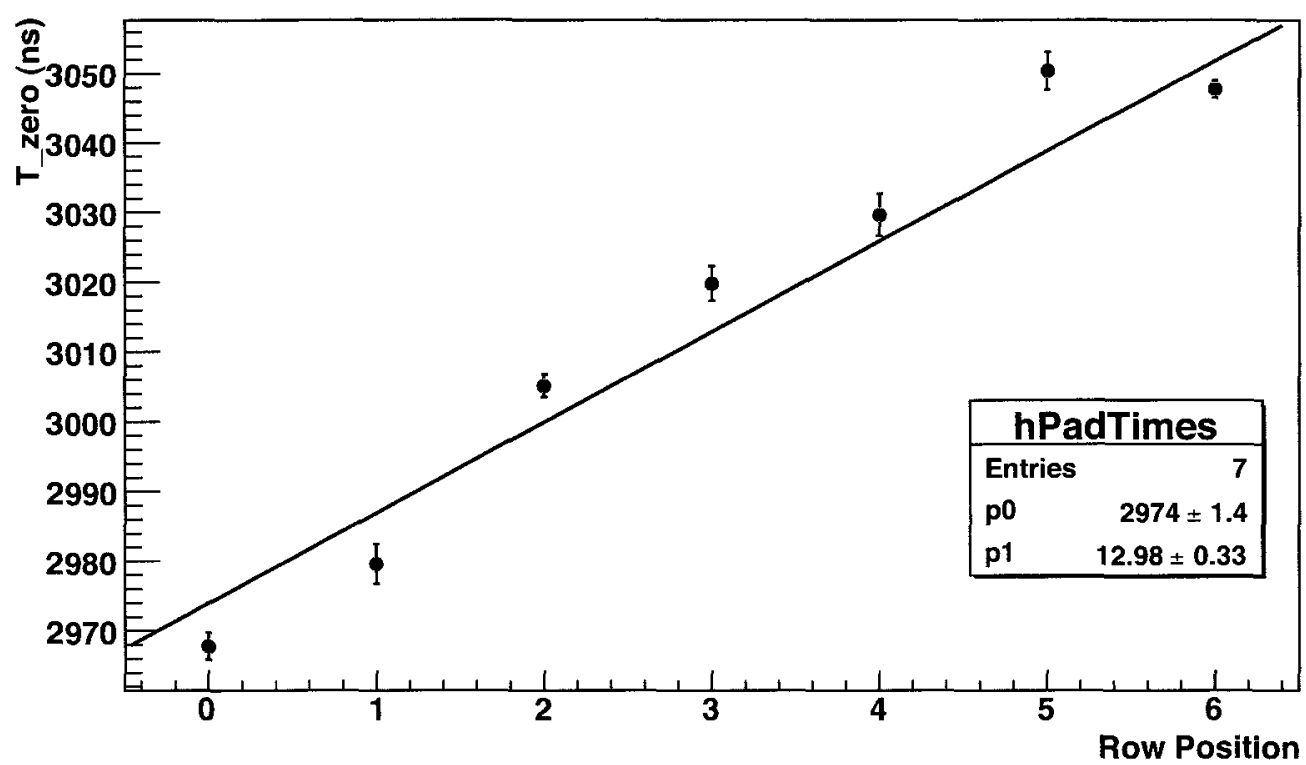

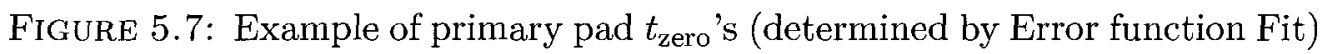
plotted against row position, fitted by least squares straight line (DESY, Run 6007 , Event $\left.11, z=8.1 \mathrm{~cm}, \theta=-9.9^{\circ}\right)$.

\subsubsection{Start Time}

The track time is taken as the fitted time for the center pad row, which is the center of the track along the $y$-axis. However, because there is some fixed time delay between the event trigger and when the FADCs begin digitizing, an offset time, $t_{\text {start }}$, is required to determine the actual drift time. This offset is found by entering all track times from a run into a histogram. Tracks from an entire run will sample the full volume of the TPC, thus $t_{\text {start }}$ is determined by the minimum track times in the distribution, as shown in Figures 5.8 and 5.9. The correct drift time for a given track is therefore: $t_{\text {drift }}=t_{\text {zero }}($ center row $)-t_{\text {start }}$.

To determine $t_{\text {start }}$, the early edge of the distribution is fit with a straight line (diagonal in Figures 5.8 and 5.9). The average bin amplitude of the flat portion 

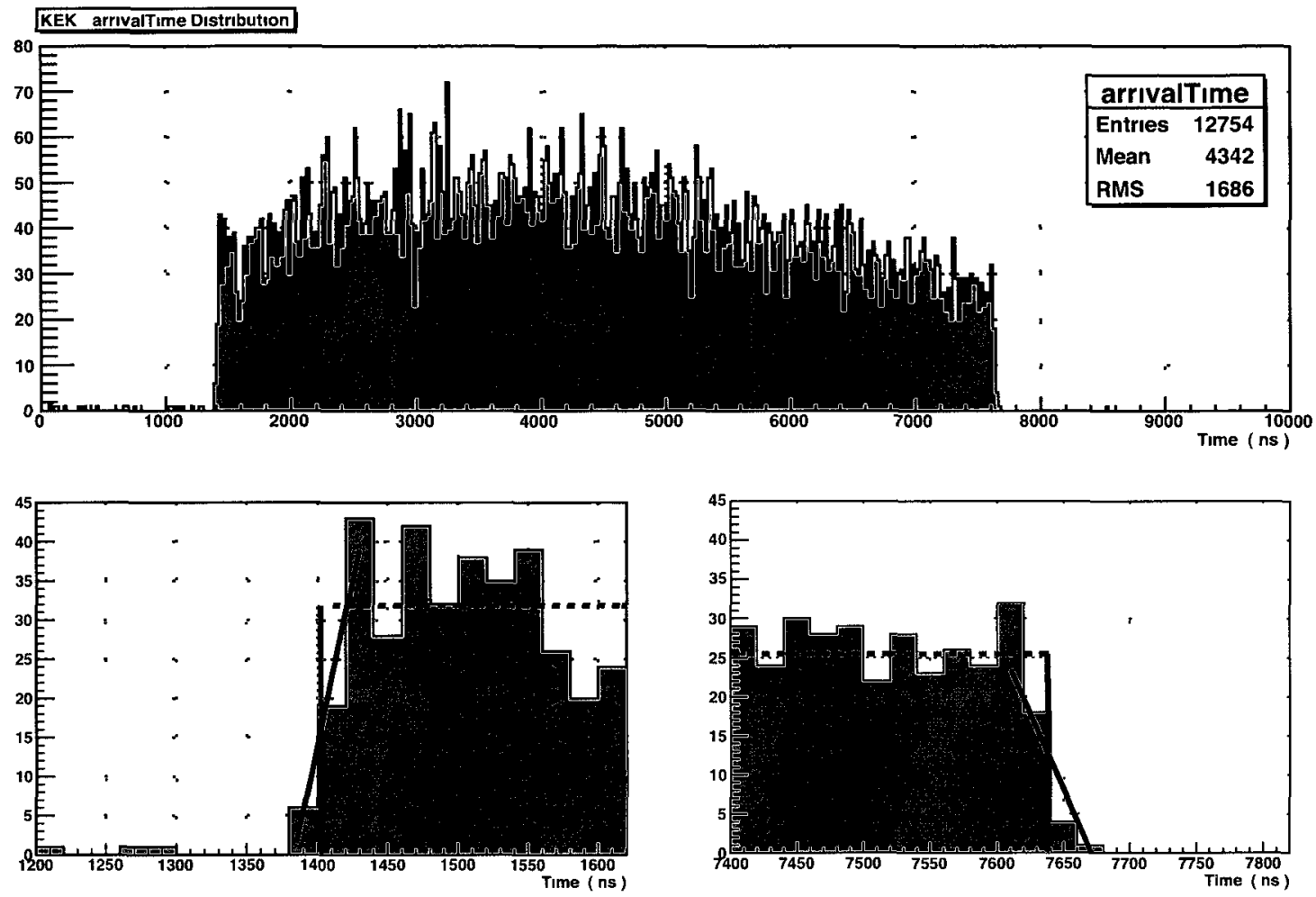

Figure 58 The arrival time $\left(t_{\text {zero }}\right)$ distribution of events from KEK, which is used to determine the drift time correction $\left(t_{\text {start }}\right)$, maximum drift time $\left(t_{\max }\right)$, and calculate the drift velocity

of the histogram (dashed blue horizontal lines) is also calculated The intersection of the fitted diagonal line with the $t$-axis, and with the dashed line, defines the early region of the distribution, which we are confident contains the true $t_{\text {start }}$ The time corresponding to the fitted edge at half the average amplitude is taken as $t_{\text {start }}$, and the uncertainty is estımated by the width of the region divided by $\sqrt{12}$ The same process is used to determine the maximum arrival time, $t_{\max }$, and the results are shown in Table 51 

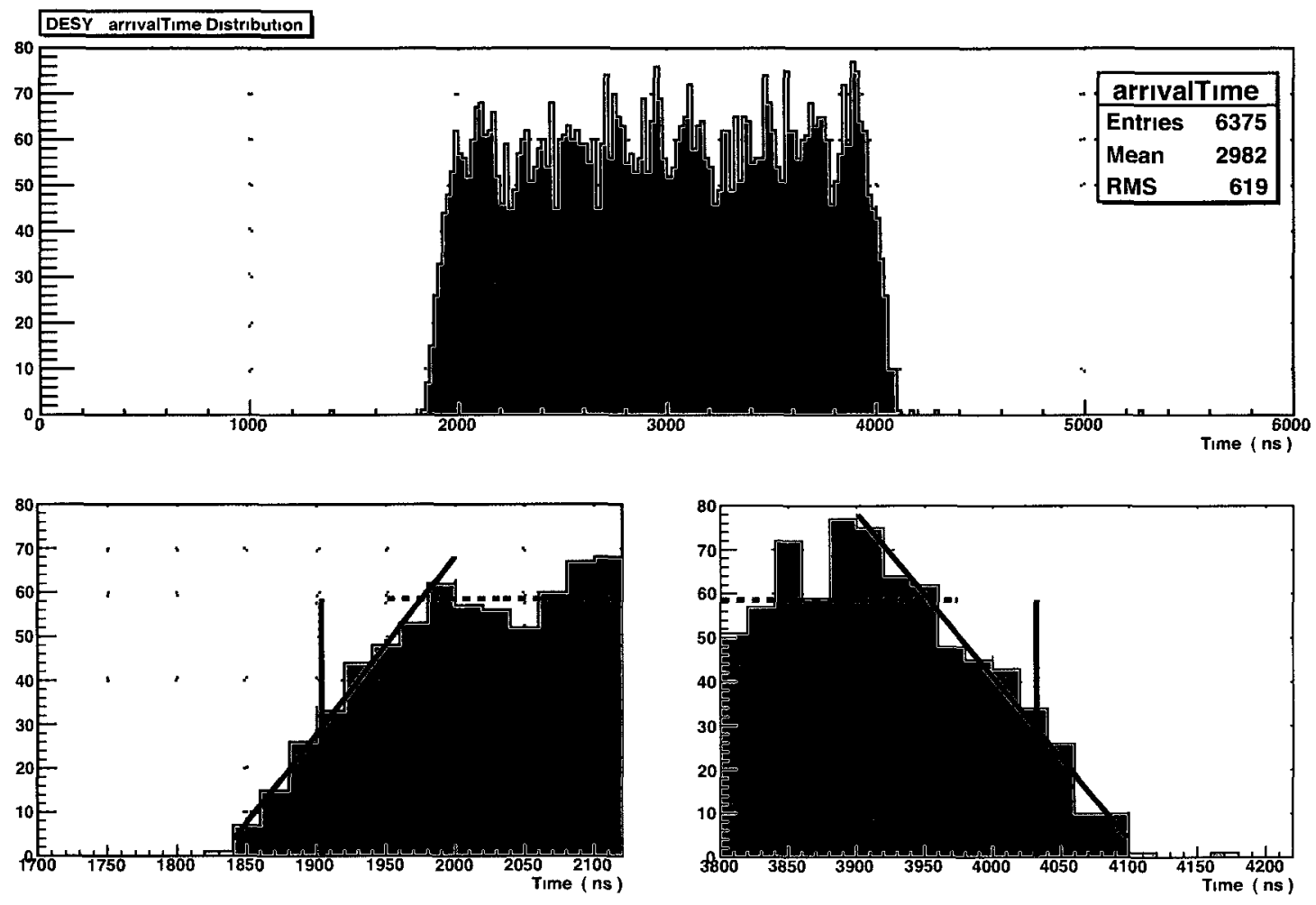

FigurE 59 The arrival time ( $\left.t_{\text {zero }}\right)$ distribution of events from DESY, which is used to determine the drift time correction $\left(t_{\text {start }}\right)$, maximum drift time $\left(t_{\max }\right)$, and calculate the drift velocity

\begin{tabular}{lcc}
\hline Experiment & $\begin{array}{c}t_{\text {start }} \\
(\mathrm{ns})\end{array}$ & $\begin{array}{c}t_{\max } \\
(\mathrm{ns})\end{array}$ \\
\hline KEK & $1402 \pm 11$ & $7638 \pm 19$ \\
DESY & $1903 \pm 41$ & $4030 \pm 47$ \\
\hline
\end{tabular}

TABLE 51 Drift time distribution analysis results 


\subsubsection{Drift Velocity}

To measure the drift velocity, we use the fact that the maximum time, $t_{\max }$, in the arrival time distribution must correspond to tracks which drifted the furthest in the TPC. The drift velocity can then be calculated as:

$$
v_{\mathrm{drift}}=\frac{l}{t_{\max }-t_{\mathrm{start}}}
$$

where $l$ is length of the TPC drift region $(157 \mathrm{~mm})$. From the KEK arrival time distribution, we find $v_{\text {drift(KEK) }}=25.2 \frac{\mu \mathrm{m}}{\mathrm{ns}}$. For DESY, we find $v_{\mathrm{drIft}(\mathrm{DESY})}=73.8 \frac{\mu \mathrm{m}}{\mathrm{ns}}$. The uncertainty in the drift velocity is derived using error propagation, which yields:

$$
\sigma_{v_{\mathrm{drift}}}=\frac{v_{\mathrm{drrft}}}{t_{\max }-t_{\mathrm{start}}} \sqrt{\sigma_{t_{\max }^{2}}^{2}+\sigma_{t_{\mathrm{start}}}^{2}}
$$

The measurements for KEK and DESY experiments are given in Table 5.2. This is a somewhat crude measurement of drift velocity as systematic errors have not been accounted for in the uncertainties. Larger TPCs are typically equipped with a laser calibration system to achieve more reliable measurements. Nonetheless, the results of the simple method used here are in agreement with the drift velocity as calculated by the Magboltz ${ }^{4}$ simulation[6], as given in Table 5.2.

\footnotetext{
${ }^{4}$ The Magboltz calculated drift velocity curves can be seen in Appendix B.
} 


\begin{tabular}{lccc}
\hline \multirow{2}{*}{ Experiment } & $E_{\text {drift }}$ & \multicolumn{2}{c}{$v_{\text {drift }}$} \\
\cline { 3 - 4 }$(\mathrm{V} / \mathrm{cm})$ & $(\mu \mathrm{m} / \mathrm{ns})$ & $\begin{array}{c}\text { Magboltz } \\
(\mu \mathrm{m} / \mathrm{ns})\end{array}$ \\
\hline KEK & 70 & $25.2 \pm 0.1$ & 26.8 \\
DESY & 200 & $74 \pm 2$ & 73.5 \\
\hline
\end{tabular}

TABLE 5.2: Drift velocity measurement results.

\subsubsection{Track Reconstruction}

With the drift velocity and start time, the track angle, $\theta$, and $z$ position can be calculated along with their uncertainties. The angle is found from:

$$
\begin{aligned}
\theta & =\arctan \left(\frac{v_{\mathrm{druft}}}{h} B \cos \phi\right) \\
\sigma_{\theta} & =\frac{\sqrt{\left(v_{\mathrm{drrft}} \cos \phi \sigma_{B}\right)^{2}+\left(B \cos \phi \sigma_{v_{\mathrm{dr} \text { rt }}}\right)^{2}+\left(v_{\mathrm{drift}} B \sin \phi \sigma_{\phi}\right)^{2}}}{h\left(1+\left(\frac{v_{\mathrm{drnft}}}{h} B \cos \phi\right)^{2}\right)}
\end{aligned}
$$

where $h$ is the pad height, and the angle $\phi$ is obtained from the transverse resolution analysis. The track height, $z$, measured from the center of the track along the $y$-axis, is calculated as

$$
\begin{aligned}
z & =v_{\mathrm{drift}}\left(y_{\text {centre }} B+A-t_{\mathrm{start}}\right) \\
\sigma_{z} & =\sqrt{\left(z \sigma_{v_{\mathrm{drrft}}}\right)^{2}+v_{\mathrm{drlft}}^{2}\left[\left(y_{\text {centre }} \sigma_{B}\right)^{2}+\sigma_{A}^{2}+\sigma_{t_{\mathrm{start}}}^{2}\right]},
\end{aligned}
$$

where $\mathrm{A}$ and $\mathrm{B}$ are the track fit parameters. The results of the track reconstruction are histogrammed in Figures 5.10 and 5.11. 


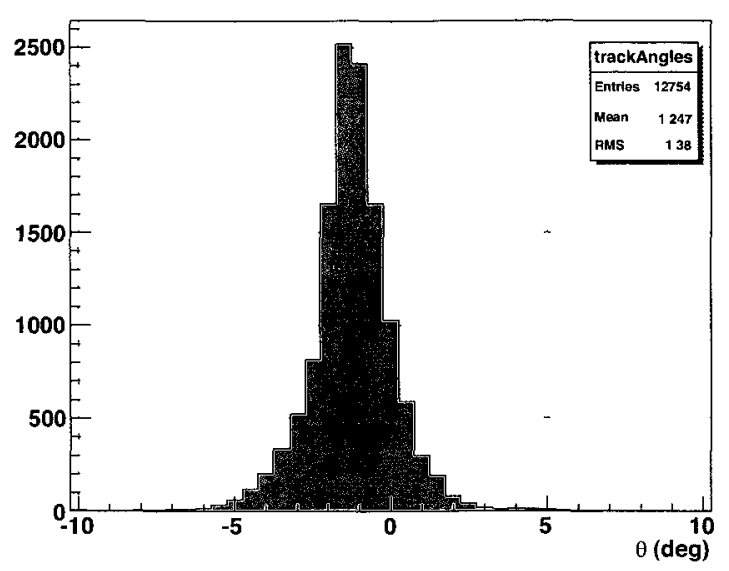

(a)

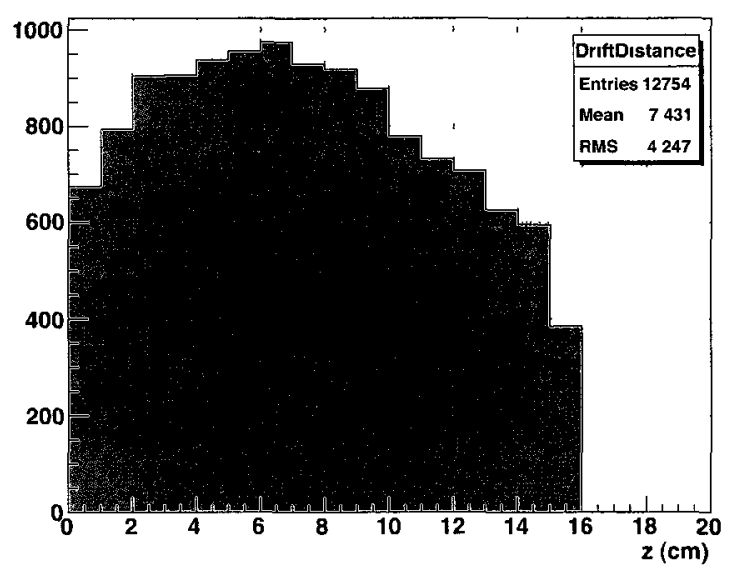

(b)

FIGURE 5.10: Distribution of track parameters $\theta$ and $z$ calculated from track reconstruction of KEK test beam of $4 \mathrm{GeV} \pi^{+}, \mathrm{B}=1 \mathrm{~T}$.

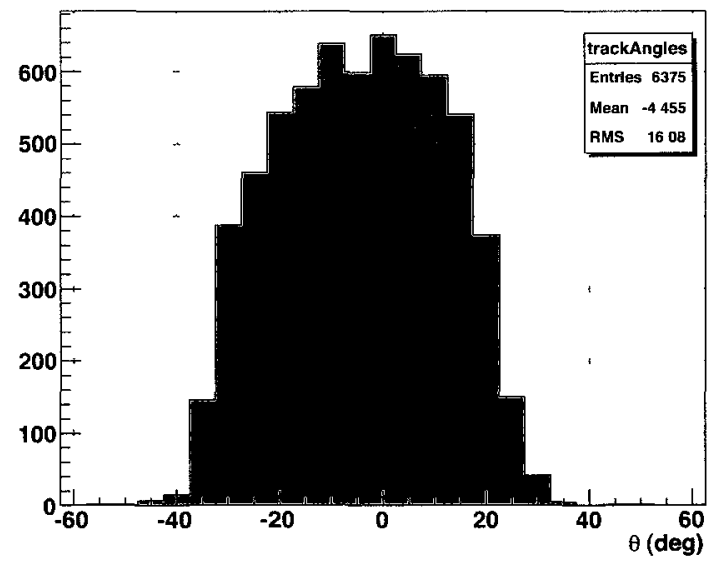

(a)

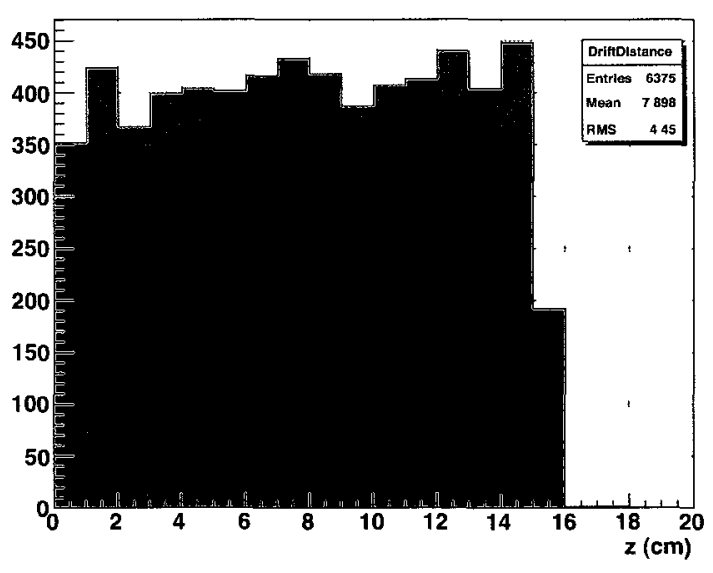

(b)

Figure 5.11: Distribution of track parameters $\theta$ and $z$ calculated from track reconstruction of DESY cosmic rays, $\mathrm{B}=5 \mathrm{~T}$. 


\subsection{Cuts}

\subsubsection{Track Angle}

Cuts based on track angle $\phi$ have already been made by the previous transverse resolution analysis, to reflect the types of events that are most interesting in actual beam collision experiments - high transverse momentum tracks. Often referred to as stiff tracks, they curve less in the magnetic field of the TPC, and therefore make smaller angles $(\phi)$ in the $\mathrm{x}-\mathrm{y}$ plane with respect to the readout pads (recall that the ILD-TPC has radial pads, rather than the rectangular readout of the COSMo TPC). The cut chosen for both the DESY and KEK analysis was $|\phi| \leq 3^{\circ}$.

\subsubsection{Edge Events}

The area covered by resistive film is larger than the area covered by pads. This leads to a problem where certain tracks that do not pass directly above the readout area would still be counted because the charge deposited on the resistive film spreads over pads nearby. In such cases, the fitting algorithm would treat these secondary pulses as primary pulses, which, because of the different characteristic shape and lower signal-to-noise ratio, would lead to poor fit results that degrade the resolution measurement. Therefore, the decision was made to cut events for 
which the primary pulse of the middle row is on an edge pad (Pads 79 and 15 in Figure 3.7$)^{5}$.

\subsubsection{Overflow Pulses}

The 8-bit FADCs can store values from 0 to 255 , so the pre-amplifier pedestals are tuned to $\sim 220$, allowing for pulses with a maximum amplitude of -220 counts, and 35 counts for undershoot. When the pulse amplitude exceeds this range, the FADC count is set to maximum. Such pulses are flagged as overflow. Timing information cannot be reliably extracted from overflow pulses because part of the peak has been chopped off, leading to poor fit results that would degrade the resolution. Therefore, the analysis program identifies each overflow pulse in an event, and these pulses are discarded from the timing analysis.

\subsection{Longitudinal Diffusion Constant}

The rise-time of primary pulses, $t_{\mathrm{rise}}$, depends on intrinsic characteristics of the detector, such as the gap width of the micromegas, characteristics of the gas, and readout electronics. However, $t_{\text {rise }}$ also depends on the elapsed time over which charge is collected by the pad - the longitudinal diffusion width, $\sigma_{D_{\mathrm{L}}}$. As discussed in Chapter 2, this depends on the drift distance, $z$, and the longitudinal diffusion

\footnotetext{
${ }^{5}$ This is sufficient because the small $\phi$ angles allowed by the cut guarantee that if the center row primary pad is not on the edge of the readout, then the track must have passed entirely over the readout pads.
} 
constant, $D_{\mathrm{L}}$. Therefore $t_{\text {rise }}$ should be proportional to the longitudinal width, $\sigma_{\mathrm{L}}$, which we interpret as a characteristic rise-time of the pulse:

$$
t_{\text {rise }} \propto \sigma_{\mathrm{L}}=\sqrt{\sigma_{\mathrm{L}_{0}}^{2}+\frac{D_{\mathrm{L}}^{2} z}{v_{\mathrm{drIft}}^{2}}},
$$

where $\sigma_{\mathrm{L}_{0}}$ is zero drift distance longitudinal width, and can be interpreted as the minimum rise-time due to intrinsic properties of the detector ${ }^{6}$.

Each time we fit a primary pulse, using either the Error Function Fit Method or the Differentiation Method, we not only obtain the arrival time $\left(t_{\text {zero }}\right)$, but also a measurement of $\sigma_{\mathbf{L}}$. By plotting $\sigma_{\mathbf{L}}$ against the drift distance $z$, and knowing the drift velocity, we can fit for the longitudinal diffusion constant, $D_{\mathrm{L}}$. However, because Equation 5.15 does not include the effect of charge dispersion, it is an only an approximation.

\subsection{Resolution}

A detector's accuracy in reconstructing a single track point is quantified by the single-hit resolution. Ideally, this would be measured by comparing the reconstructed track points to the known track position. The residuals are defined as the difference between the reconstructed point, $t_{\text {zero }}$, and the true track position,

\footnotetext{
${ }^{6}$ The $\theta$ angle will also effect $t_{\text {rise }}$, and will be explored in Chapter 6 .
} 
$t_{\text {track: }}$

$$
R=t_{\text {track }}-t_{\text {zero }} .
$$

These residuals, in the absence of systematic bias, are Gaussian distributed, and the uncertainty in each $t_{\text {zero }}$ measurement is the standard deviation of this distribution. This is known as the single-hit resolution. Our tests of the COSMo TPC, however, did not include an external reference for the particle trajectories through the chamber, so the actual positions of track points are not known. Therefore, the resolution can only be estimated from the internal consistency of the data. The solution we have used is known as the Geometric Mean Method.

\subsubsection{The Geometric Mean Method}

This method calculates the resolution from the geometric mean of standard deviations of residuals from track fits done in two different ways: including and excluding the row for which the residual is calculated. For each event, the leastsquares track fit over all rows is used to calculate an inclusive residual for each pad row:

$$
R_{\imath}^{\mathrm{INC}}=t_{\mathrm{fit}_{1}}-t_{\mathrm{zero}_{1}} \quad(\imath=1,2, \ldots, 7) .
$$

The 7 residuals are added to the inclusive histogram. Next, seven additional track fits are performed for the same event, each with a different single point, $t_{\text {zero, }}$ 
removed from the fit, to calculate the exclusive residuals:

$$
R_{\jmath}^{\mathrm{EXC}}=t_{\mathrm{fit}_{\mathrm{t}}}^{*}-t_{\mathrm{zero}_{\mathrm{J}}} \quad(\jmath=1,2, \ldots, 7)
$$

These 7 residuals are added to the exclusive histogram. This processes is illustrated by Figure 5.12. The best estimate of the longitudinal single-hit resolution is then given by:

$$
\epsilon_{L}=\sqrt{\sigma_{\mathrm{INC}} \cdot \sigma_{\mathrm{EXC}}}
$$

which is the geometric mean of the standard deviations of the inclusive and exclusive residual distributions. The derivation of this method comes from [19], and is included in Appendix E.

The motivation behind taking the resolution as the geometric mean can be thought of as follows: the inclusive residuals are expected to be smaller than the actual residuals, because the reconstructed points are correlated with the fitted track position - the points pull the fitted track towards them. Therefore the distribution width of inclusive residuals, $\sigma_{\mathrm{INC}}$, is too narrow, and an over estimate of the resolution. On the other hand, the exclusive residuals are expected to be too large. The absence of the reconstructed point in the least-squares fit allows the other points, which themselves have uncertainty, to pull the fitted track away from the true track position. Therefore, the exclusive residual distribution, $\sigma_{\mathrm{EXC}}$, is wider than the true residual distribution, giving an underestimate of the resolution. 


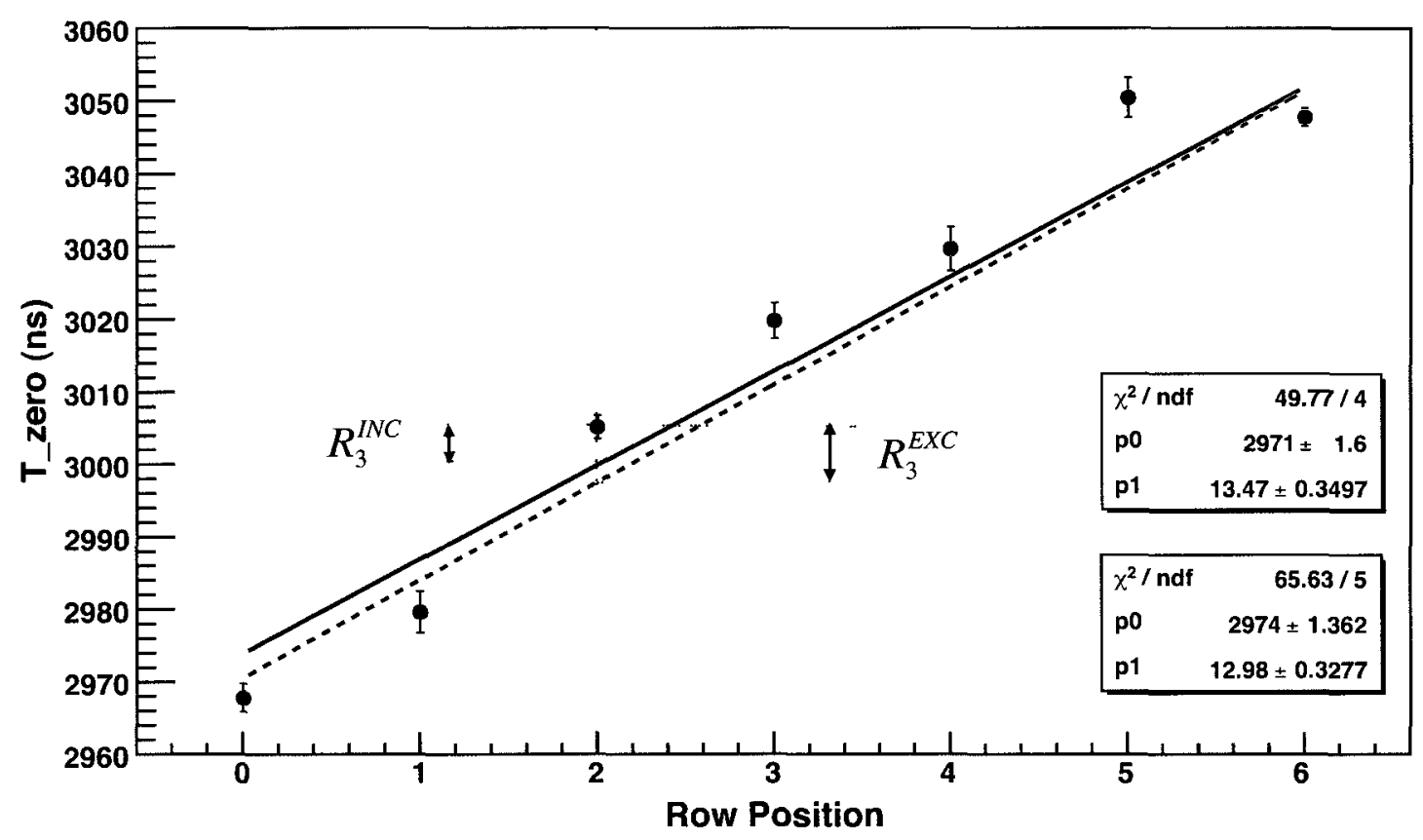

FigURE 5.12: Illustration of how residuals are calculated to measure the resolution. The solid line (black) is the weighted least-squares fit to all 7 data points, from which the inclusive residuals, $R^{I N C}$ are calculated. The dashed line (red) is one of the 7 additional fits, each with a different single point removed. In this example, the 3 rd data point has been removed from the fit, which yields $R_{3}^{E X C}$. 


\section{Chapter 6}

\section{Results}

As previously mentioned, the raw pulses used in the analysis have 5 ns wide bins, a result of the extremely fast $200 \mathrm{MHz}$ FADCs in the COSMo TPC. To demonstrate that this speed is not necessary, the raw data has been re-binned to 25 and $40 \mathrm{~ns}$, which correspond to effective sampling rates of 40 and $25 \mathrm{MHz}$ respectively for comparison, as slower FADCs are less expensive. Measurements of longitudinal resolution were carried out for all 3 bin widths. The plots displayed in this chapter are for 25 ns bins, as this is the most likely choice for the ILDTPC. All plots in this chapter were generated using the Error function fit method of timing. Measurements using the 5-point numerical differentiation method are included in the summary tables for comparison. 


\subsection{Longitudinal Diffusion}

As described in Chapter 5, each primary pulse analyzed for the charge cluster arrival time, $t_{z e r o}$, also yields a measurement of the primary pulse rise time, which should be approximately proportional to the cluster width, $\sigma_{\mathrm{L}}$, allowing us to make a rough measurement of the longitudinal diffusion constant, $D_{\mathrm{L}}$. To test this relationship, scatter plots of $\sigma_{\mathrm{L}}$ versus $z$ are displayed in Figures 6.1 and 6.2. The dashed line (blue) is the expected behaviour:

$$
\sigma_{\mathrm{L}}=\sqrt{\sigma_{\mathrm{L}_{0}}^{2}+\sigma_{\mathrm{D}_{\mathrm{L}}}^{2}},
$$

using gas parameters from Magboltz. The scatter plot from the KEK analysis shows a much stronger dependence of the primary pulse rise-time on the drift distance, compared to the relatively flat curve from the DESY analysis, which is not surprising given the different drift velocities. The DESY drift velocity $(74 \mu \mathrm{m} / \mathrm{ns})$ is approximately three times higher than the KEK drift velocity $(25 \mu \mathrm{m} / \mathrm{ns})$, which means less time for the drifting electrons to diffuse.

To measure the diffusion constant, the $\sigma_{\mathrm{L}}$ are divided into $z$-bins, and each histogram is fitted with a Gaussian to determine the mean pulse width $\left(\bar{\sigma}_{\mathrm{L}}\right)$ and standard deviation corresponding to the drift distance at the center of the bin ${ }^{1}$. Half centimetre wide $z$-bins were chosen for the KEK analysis, compared to $1 \mathrm{~cm}$

\footnotetext{
${ }^{1}$ See Appendix B for the fitted histograms.
} 
wide bins for DESY, due to the difference in the number of points in the scatter plots (76997 and 42372 respectively).

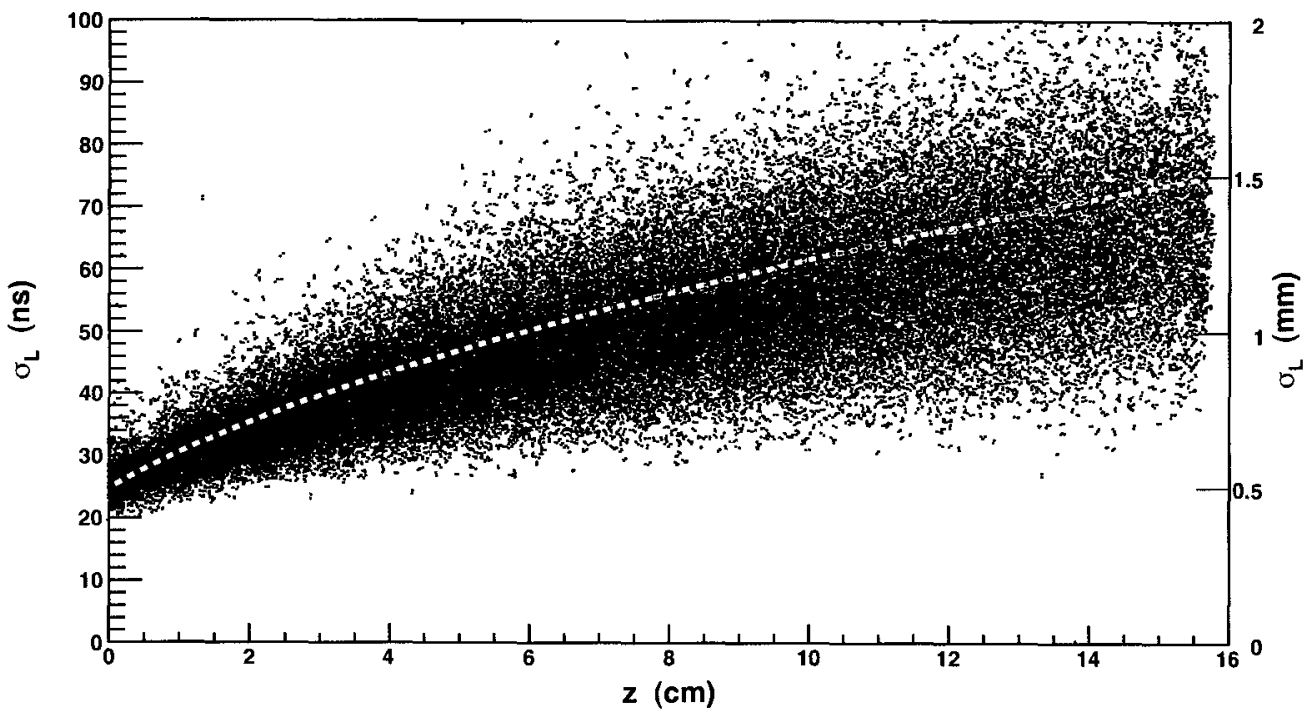

Figure 6.1: Scatter plot of pulse width versus drift distance, $z$, from the KEK analysis. The dashed line (blue) is the expected curve using the Magboltz parameters: $D_{\mathrm{L}}=479 \mu \mathrm{m} / \sqrt{\mathrm{cm}}$, and $v_{\text {drift }}=26.8 \mu \mathrm{m} / \mathrm{ns}$.

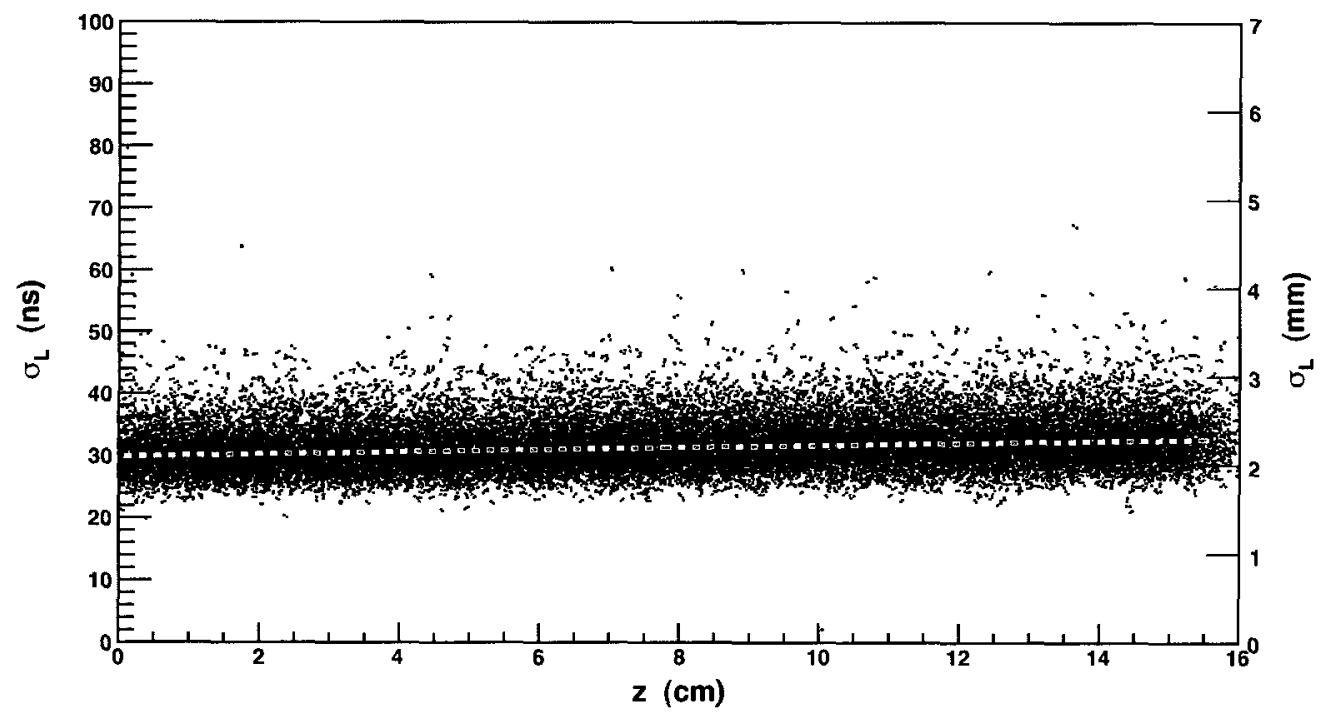

FiguRe 6.2: Scatter plot of pulse width versus drift distance, $z$, from the DESY analysis. The dashed line (blue) is the expected curve using the Magboltz parameters: $D_{\mathrm{L}}=248 \mu \mathrm{m} / \sqrt{\mathrm{cm}}$, and $v_{\text {drft }}=73.5 \mu \mathrm{m} / \mathrm{ns}$. 
The longitudinal diffusion constant is obtained from the slope, $B$, of the line:

$$
\bar{\sigma}_{\mathrm{L}}^{2}=\sigma_{\mathrm{L}_{0}}^{2}+B z
$$

which is fit to the points using weighted least-squares, where $B=D_{\mathrm{L}}^{2} / v_{\mathrm{drift}}^{2}$.

For the KEK analysis, using the error function fit timing method and 25 ns wide bins, we find that $D_{\mathrm{L}} \approx 413 \mu \mathrm{m} / \sqrt{\mathrm{cm}}$, in rough agreement with the Magboltz calculation for the diffusion constant, $479 \mu \mathrm{m} / \sqrt{\mathrm{cm}}$. Similarly for the DESY analysis, we find $D_{\mathrm{L}} \approx 259 \mu \mathrm{m} / \sqrt{\mathrm{cm}}$, which is close to the Magboltz calculated value of $248 \mu \mathrm{m} / \sqrt{\mathrm{cm}}$. So we can see that this analysis technique is sensitive to changes in cluster width, however because of charge dispersion, the fitting algorithm would need to be improved to make a precise measurement of $D_{\mathrm{L}}$.

\subsection{Resolution}

As described in Section 5.5, the single-hit longitudinal resolution, $\epsilon_{\mathrm{L}}$, is determined from the geometric mean of inclusive and exclusive distributions of track fit residuals. To examine the drift distance dependence of resolution in COSMo TPC, the analysis program directs residuals into $1 \mathrm{~cm}$ wide $z$-bins. Figures 6.3 and 6.5 show the inclusive and exclusive residual histograms of $z$-bins centred at 0.5 and 14.5 
$\mathrm{cm}$ for comparison. The greater change in width of the KEK distributions over the full TPC drift distance is expected given the large increase in pulse width observed in Figure 6.2. As the charge cloud diffuses, the uncertainty in its mean position increases, therefore the width of the track fit residual distribution increases.

With the longitudinal point resolution calculated for each of the $15 z$ regions, the zero drift resolution, $\epsilon_{\mathrm{L}_{0}}$, is found by fitting these points with the curve:

$$
\epsilon_{\mathrm{L}}=\sqrt{\epsilon_{\mathrm{L}_{0}}^{2}+\xi z}
$$

which was derived in Section 2.1.3. The parameters $\epsilon_{\mathrm{L}_{0}}$ and $\xi$ (which is equal to $\left.D_{\mathrm{L}}^{2} /\left(v_{\text {drift }}^{2} N_{\text {eff }}\right)\right)$ are determined by a $\chi^{2}$ minimization.

Figure 6.4 shows the KEK resolution plotted against drift distance. Using the Error function fit timing method and 25 ns time bins, we find $\epsilon_{\mathrm{L}_{0}}=4.07 \pm 0.08$ ns. Using the drift velocity and its uncertainty, this is equivalent to a $z$-coordinate intrinsic resolution of $\epsilon_{\mathrm{z}_{0}}=103 \pm 2 \mu \mathrm{m}$.

Figure 6.6 shows the DESY resolution, where we find $\epsilon_{\mathrm{L}_{0}}=7.2 \pm 0.1 \mathrm{~ns}$, which is equivalent to $\epsilon_{\mathrm{z}_{0}}=535 \pm 8 \mu \mathrm{m}$. The ILD-TPC requirement for intrinsic $z$-resolution is $\sim 500 \mu \mathrm{m}$, so these are good results. The measurements of $\epsilon_{\mathrm{L}_{0}}$ for other time bin widths and the 5-point numerical differentiation method are included in Table 6.1. The values measured using the error function fit method do not change significantly with re-binning the signal. 
The fit parameter $\xi$ is equal to $D_{\mathrm{L}}^{2} /\left(v_{\text {drift }}^{2} N_{\text {eff }}\right)$, where $N_{\text {eff }}$ is the effective number of electrons contributing to the resolution measurements. Resolution improves with the number of electrons contributing to the measurement, analogous to how the accuracy of a mean improves as the number of measurements increases: the standard deviation of the mean. To calculate the effective number of electrons and uncertainty we have:

$$
\begin{aligned}
N_{\text {eff }} & =\frac{D_{\mathrm{L}}^{2}}{v_{\text {drift }}^{2} \xi}, \\
\sigma_{\mathrm{N}_{\text {eff }}} & =\frac{D_{\mathrm{L}}}{v_{\mathrm{drift}}^{2} \xi} \sqrt{\left(2 \sigma_{D_{\mathrm{L}}}\right)^{2}+\left(\frac{2 D_{\mathrm{L}} \sigma_{v_{\mathrm{drift}}}}{v_{\text {drift }}}\right)^{2}+\left(\frac{D_{\mathrm{L}} \sigma_{\xi}}{\xi}\right)^{2}} .
\end{aligned}
$$

which gives a value of approximately 10 electrons for both KEK and DESY, independent of time bin size when using the error function fit method. All results of the $N_{\text {eff }}$ measurements are shown in Table 6.2.

With $\epsilon_{\mathrm{L}_{0}}$ and $N_{\text {eff }}$ and the diffusion constant, we can extrapolate the expected resolution to longer drift distances. For the maximum drift of the ILD-TPC, using the T2K gas from DESY, we could expect $\epsilon_{\mathrm{L}}(z=200 \mathrm{~cm}) \approx 17 \mathrm{~ns}$, or 1300 $\mu \mathrm{m}$, consistent with the ILD-TPC requirement of $1400 \mu \mathrm{m}$, as shown in Figure 6.7 . 


\subsubsection{KEK Results}
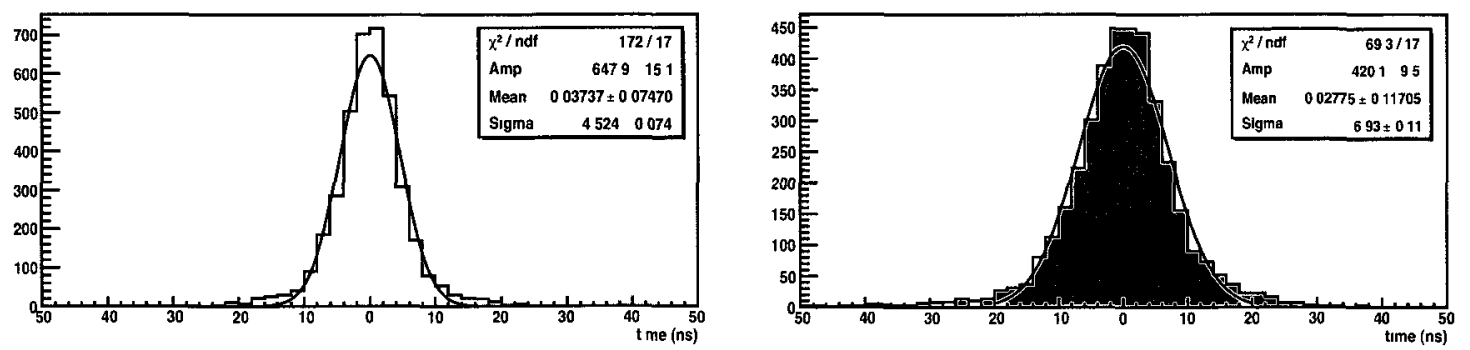

(a) $0 \mathrm{~cm}<z<1 \mathrm{~cm}$
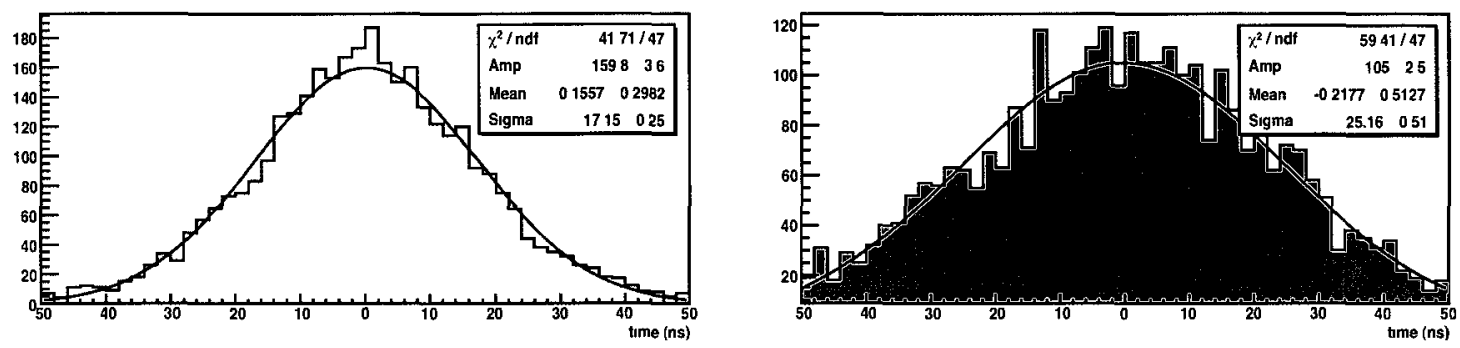

(b) $14 \mathrm{~cm}<z<15 \mathrm{~cm}$

FIGURE 63 Sample of KEK inclusive and exclusive residual distributions

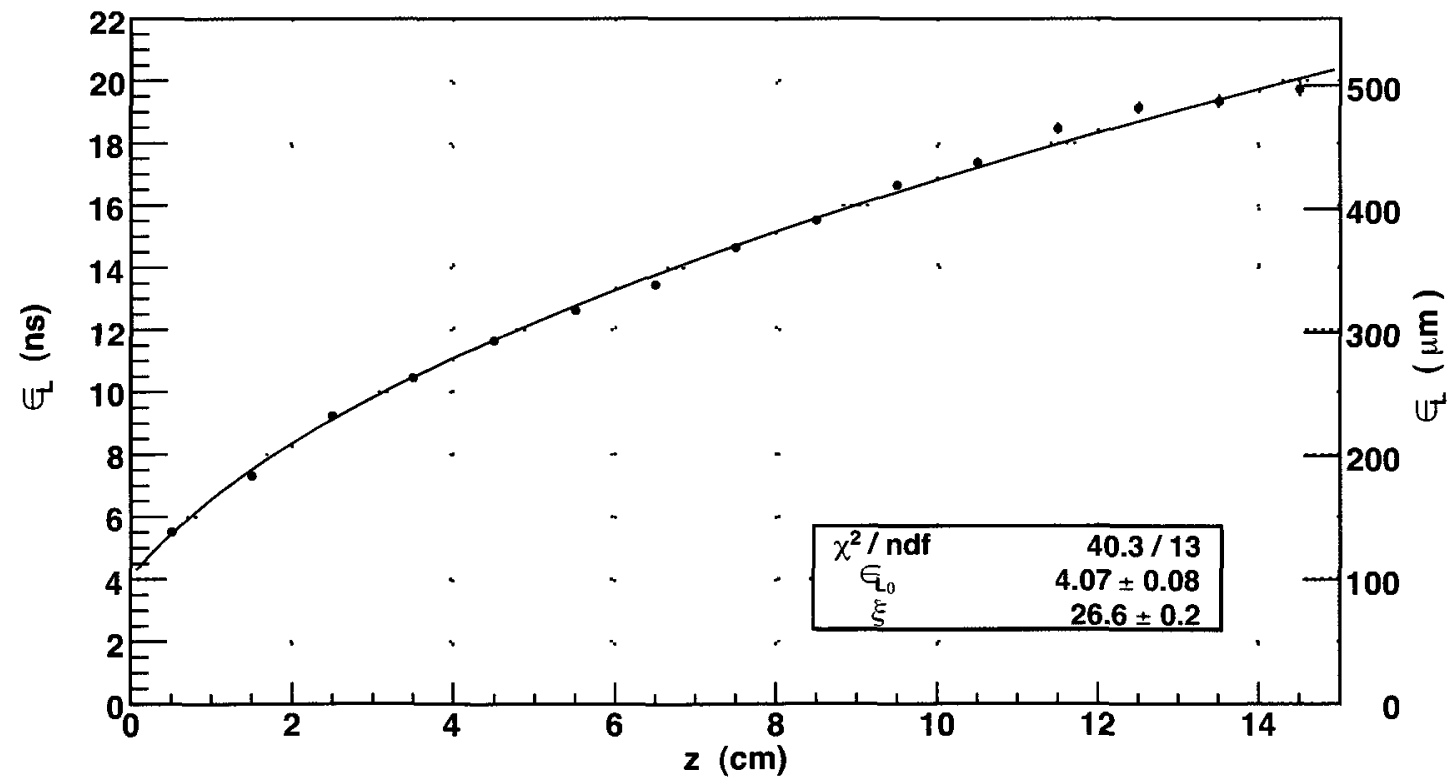

FIgURE 64 KEK Resolution dependence on $z$ (Error function Fit timing, 25 ns time bins) 


\subsubsection{DESY Results}
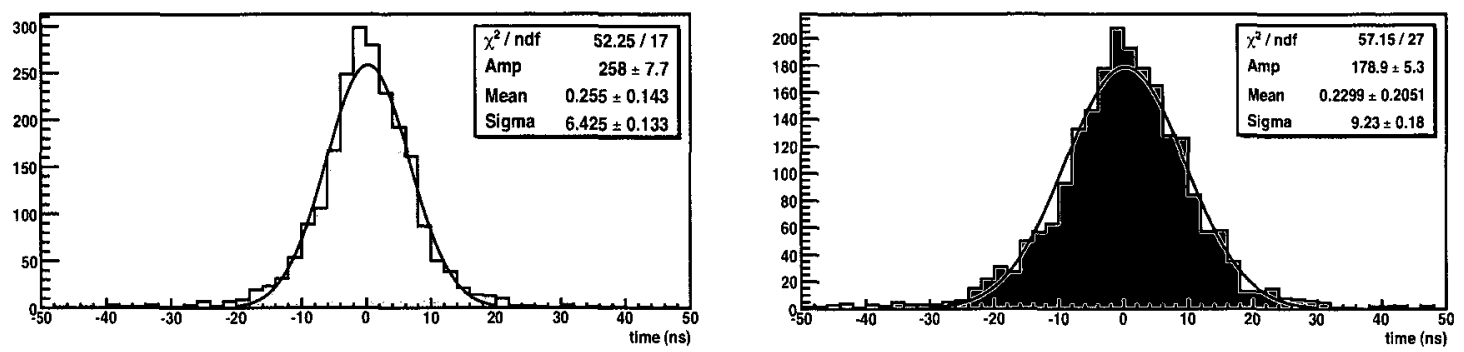

(a) $0 \mathrm{~cm}<z<1 \mathrm{~cm}$
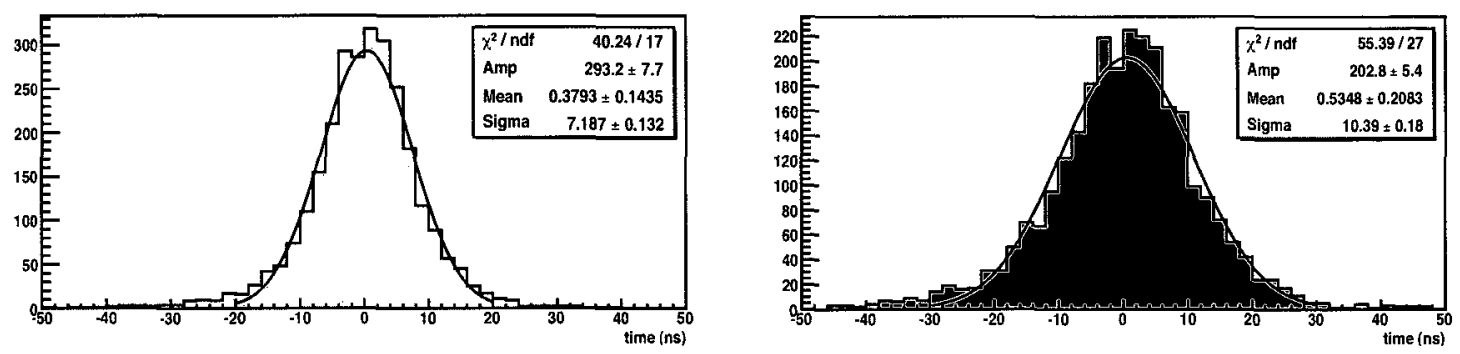

(b) $14 \mathrm{~cm}<z<15 \mathrm{~cm}$

FIGURE 6.5: Sample of DESY inclusive and exclusive residual distributions.

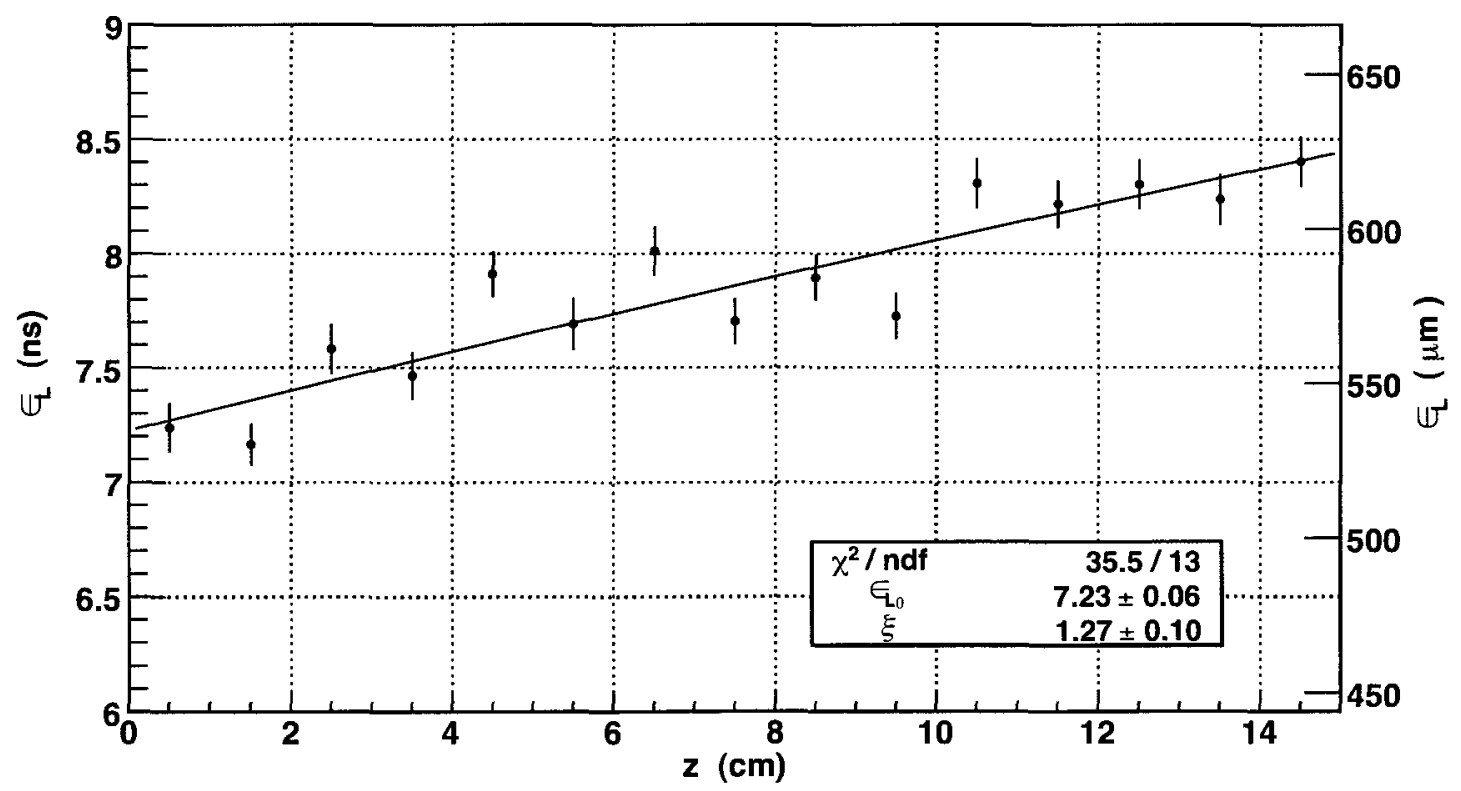

FIGURE 6.6: DESY Resolution dependence on drift distance, $z$. (Error fucntion

Fit timing, 25 ns bins) 


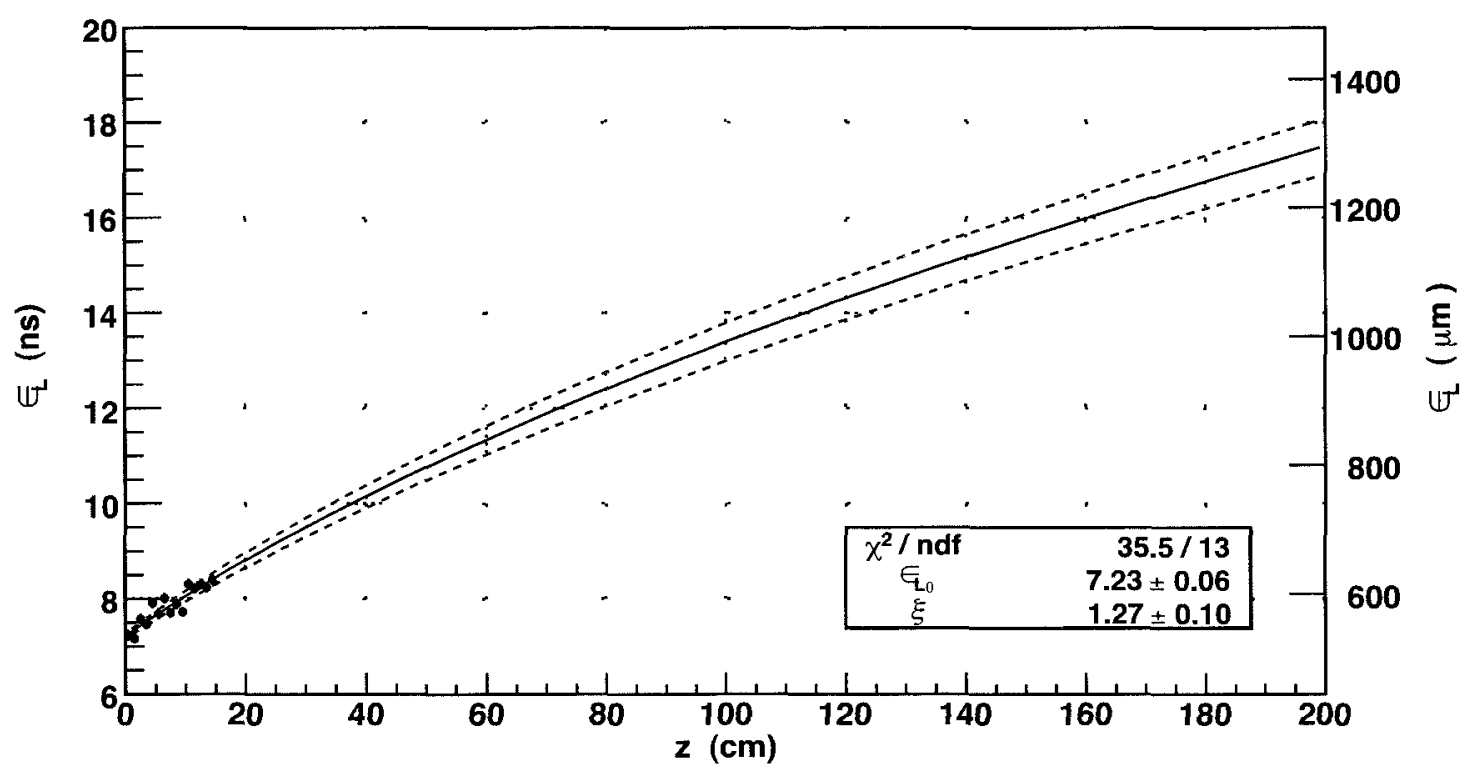

FIGURE 6.7: DESY Resolution dependence on drift distance from Figure 66 extrapolated to the expected $200 \mathrm{~cm}$ drift length of the ILD-TPC. The dashed curves (red) are calculated using the 1-sigma uncertainty in fit parameters. The extrapolated resolution is below the $1400 \mu \mathrm{m}$ requirement of the ILD-TPC.

\subsubsection{Summary}

\begin{tabular}{lcccc}
\hline \multirow{2}{*}{ Experiment } & \multirow{2}{*}{$\mathbf{t}_{\text {zero }}$} & \multicolumn{3}{c}{$\epsilon_{\mathrm{L}_{0}}(\mathrm{~ns})$} \\
\cline { 3 - 5 } & & \multicolumn{3}{c}{ time bin width } \\
\cline { 3 - 5 } KEK & Error Function Fit & $3.9 \pm 0.1$ & $4.1 \pm 0.1$ & $4.2 \pm 0.1$ \\
& 5-pt Differentiation & $3.5 \pm 0.1$ & $4.3 \pm 0.1$ & $5.0 \pm 0.1$ \\
DESY & & & & \\
& Error Function Fit & $7.2 \pm 0.1$ & $7.2 \pm 0.1$ & $7.5 \pm 0.1$ \\
& 5-pt Differentiation & $7.1 \pm 0.1$ & $7.4 \pm 0.1$ & $7.5 \pm 01$ \\
\hline
\end{tabular}

TABLE 6.1: Measurements of the intrinsic longitudinal resolution, $\epsilon_{\mathrm{L}_{0}}$. 


\begin{tabular}{lcccc}
\hline \multirow{2}{*}{ Experiment } & \multirow{2}{*}{$\mathbf{t}_{\text {zero }}$} & \multicolumn{3}{c}{$\mathbf{N}_{\text {eff }}$} \\
\cline { 3 - 5 } & & $5 \mathrm{~ns}$ & time bin width \\
& & $25 \mathrm{~ns}$ & $40 \mathrm{~ns}$ \\
\hline KEK & Error Function Fit & $10.0 \pm 0.1$ & $10.1 \pm 0.1$ & $10.2 \pm 0.1$ \\
& 5-pt Differentiation & $7.4 \pm 0.1$ & $7.9 \pm 0.1$ & $8.1 \pm 0.1$ \\
\multirow{2}{*}{ DESY } & & & & \\
& Error Function Fit & $10.5 \pm 1.0$ & $9.6 \pm 0.9$ & $9.7 \pm 1.0$ \\
& 5-pt Differentiation & $8.3 \pm 0.8$ & $9.7 \pm 1.0$ & $7.3 \pm 0.7$ \\
\hline
\end{tabular}

TABLE 6.2: Measurements of the effective number of electrons. $N_{\text {eff }}$.

\subsection{Resolution Dependence on $\theta$}

As has been shown, the width of the charge cloud distribution in the longitudinal direction depends on diffusion, $\sigma_{D_{L}}$. There is however an additional contribution to the width, $\sigma_{\theta}$, which is a geometrical spread of the charge cluster above a pad due to the angle between the particle momentum and the cluster drift direction:

$$
\sigma_{\theta}=\frac{L_{p a d} \tan \theta}{v_{\text {drift }} \sqrt{12}}
$$

where $L_{\mathrm{pad}} \tan \theta$ is the projected track segment length in the $z$-direction (assuming ${ }^{2}$ $\phi \approx 0$ ), and the $\sqrt{12}$ factor comes from the variance of uniformly distributed electrons along the track [20]. Therefore, the total longitudinal variance can be ${ }^{2}$ If $\phi \neq 0$, then $L_{\text {pad }} \rightarrow L_{\text {pad }} / \cos \phi$. 


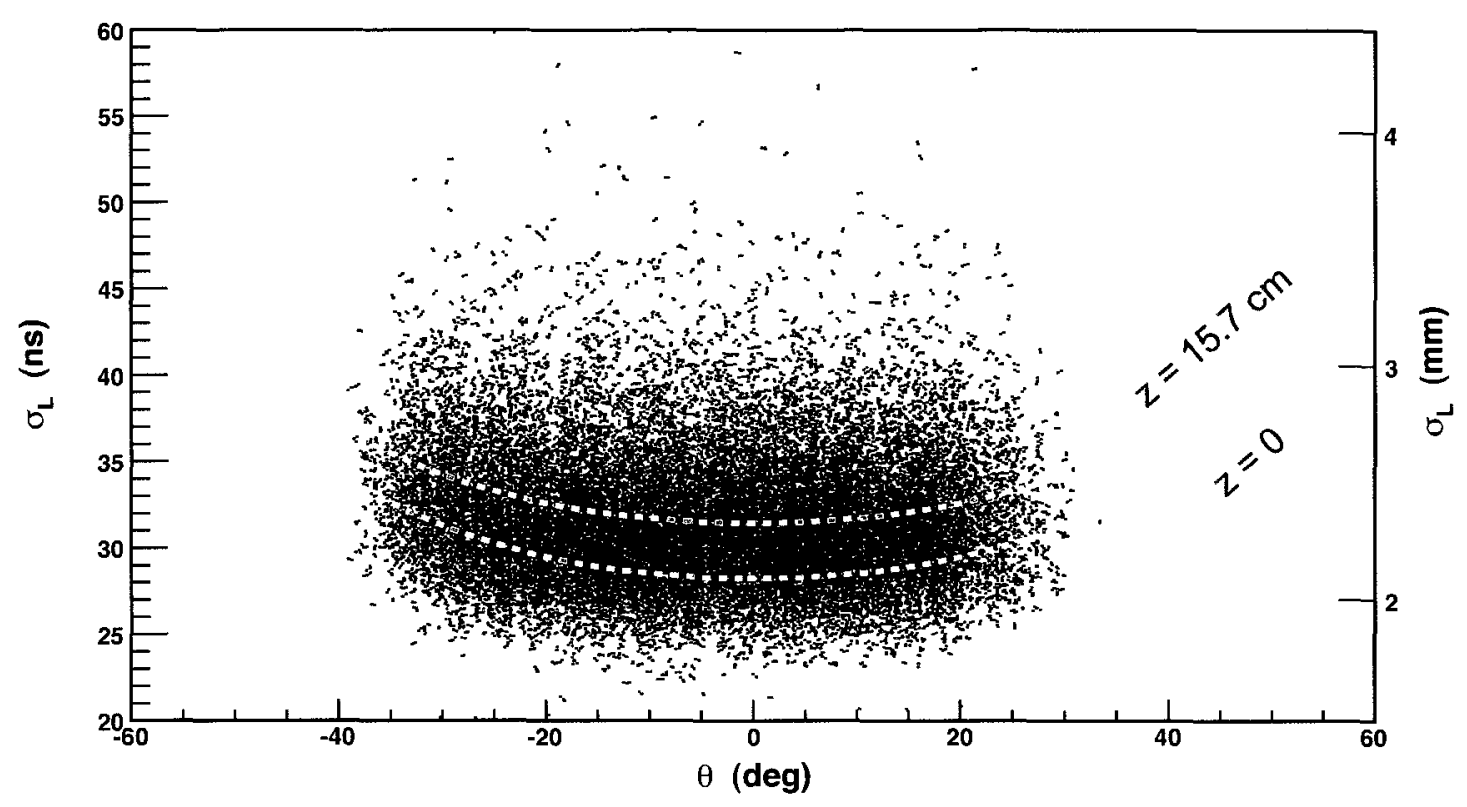

FiguRE 6.8: DESY pulse width dependence on track angle The dashed lines are calculated from Equation 6.7 to show the contribution of diffusion.

estimated as:

$$
\begin{aligned}
& \sigma_{\mathrm{L}}^{2}(z, \theta)=\sigma_{\mathrm{L}_{0}}^{2}+\sigma_{D_{\mathrm{L}}}^{2}+\sigma_{\theta}^{2}, \\
& \sigma_{\mathrm{L}}(z, \theta)=\sqrt{\sigma_{\mathrm{L}_{0}}^{2}+\frac{D_{\mathrm{L}}^{2} z}{v_{\mathrm{drfft}}^{2}}+\left(\frac{L_{p a d} \tan \theta}{v_{\mathrm{drift}} \sqrt{12}}\right)^{2}},
\end{aligned}
$$

The DESY experiment data contans events with a relatively large range of $\theta$ angles $\left(-40^{\circ}<\theta<30^{\circ}\right)$, so the effect of track angle on resolution can also be investigated. To observe this track angle effect, a scatter plot of the pulse width against track angle ( $\sigma_{\mathrm{L}}$ vs $\theta$ ) is shown in Figure 6.8. Some of the vertical spread in the scatter plot can be accounted for by diffusion, as shown by the two dashed curves which were calculated from Equation 67 using $z=0$ and $z=15.7 \mathrm{~cm}$, the minimum and maximum drift distances. 


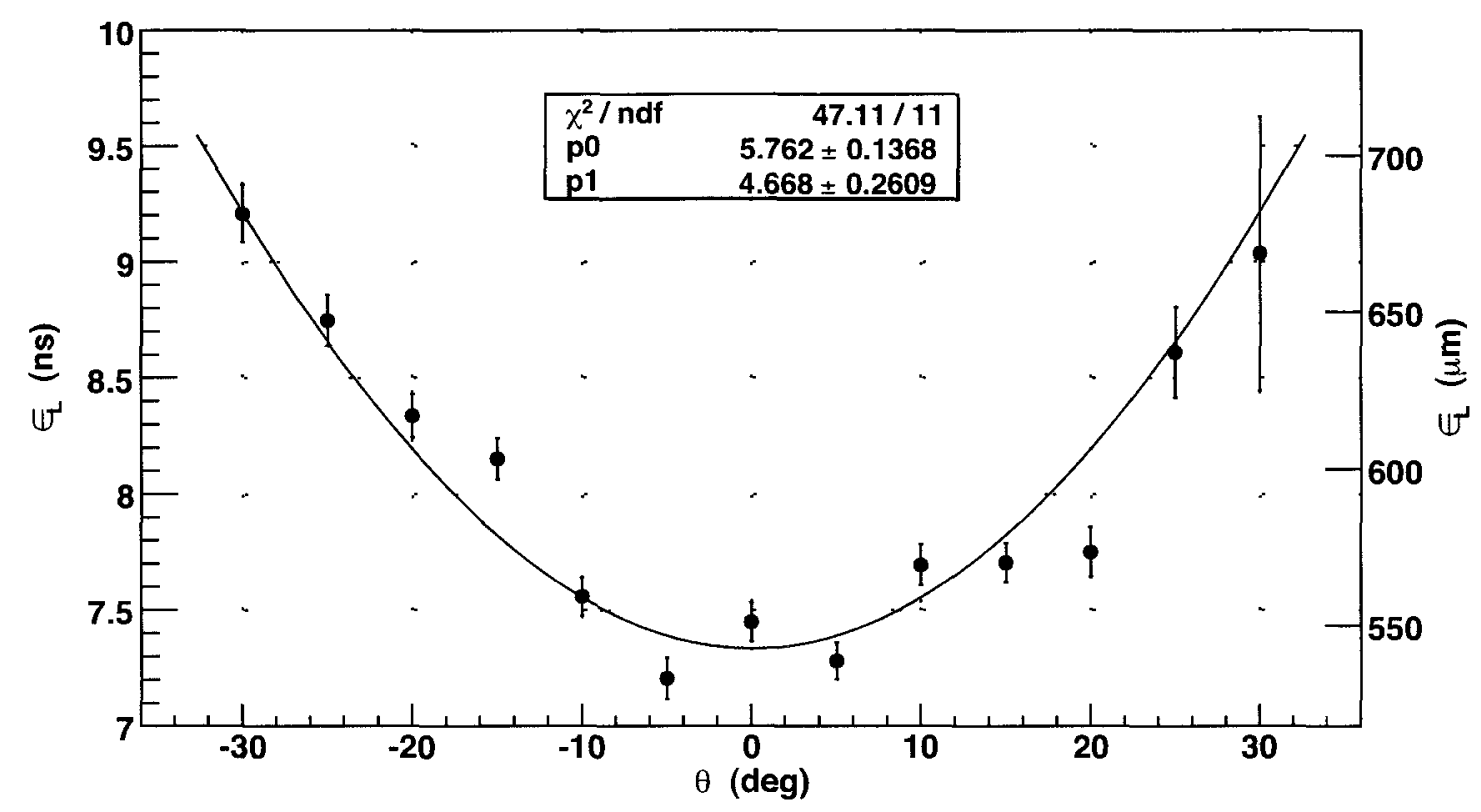

FiguRE 6.9 DESY longitudinal resolution as a function of track angle, $\theta$.

The resolution is again dependent on the effective number of electrons, which increases with the $\theta$ angle because the length of the track segment above the pad is inversely proportional to the cosine of $\theta$. Therefore, the resolution should follow:

$$
\begin{aligned}
& \epsilon_{\mathrm{L}}^{2}(\theta)=\epsilon_{\mathrm{L}_{0}}^{2}+\sigma_{D_{\mathrm{L}}}^{2} \frac{\cos \theta}{N_{\text {eff }}}+\sigma_{\theta}^{2} \frac{\cos \theta}{N_{\mathrm{eff}}} \\
& \epsilon_{\mathrm{L}}(\theta)=\sqrt{\epsilon_{\mathrm{L}_{0}}^{2}+\frac{D_{\mathrm{L}}^{2} z}{v_{\text {drift }}^{2}} \frac{\cos \theta}{N_{\text {eff }}}+\left(\frac{L_{\text {pad }} \tan \theta}{v_{\text {drift }} \sqrt{12}}\right)^{2} \frac{\cos \theta}{N_{\text {eff }}}},
\end{aligned}
$$

which is plotted in Figure 6 9, where the measured resolution has been measured in $\theta$ bins of $5^{\circ}$ width. The value of $\epsilon_{L_{0}}$ obtained from the fit in Figure 6.9 is $\approx 6 \mathrm{~ns}$, smaller than that obtained from the $\epsilon_{L}$ vs $z$ because that plot included all track angles. The values of $N_{\text {eff }}$ also differ. Therefore, the best we can conclude is that the resolution is approximately proportional to $\tan \theta$, as expected. 


\section{Chapter 7}

\section{Conclusion}

I have conducted the first analysis of the longitudinal resolution of an Micromegas TPC with charge dispersion: a proposed central tracking device for the future ILC experiment. A new timing algorithm was developed based on a fit to the leading edge of digitized charge pulses with an error function to determine the mean cluster arrival time. This new method also provides a means measuring the drift velocity and longitudinal diffusion parameters in the TPC, which serves as a consistency check with values obtained by simulation.

Data from two different tests of the prototype COSMo TPC were analyzed. In the first experiment, conducted in 2005 at KEK in Japan, the TPC was tested inside a $1 \mathrm{~T}$ magnet, using a $4 \mathrm{GeV} \pi^{+}$beam. The TPC was filled with a gas mixture of $95 \%$ argon and $5 \%$ isobutane, and had a $70 \mathrm{~V} / \mathrm{cm}$ drift field. In Chapter 5, using the new error function timing method, I measured the drift velocity 
approximately to be $v_{\text {drift }}(K E K) \approx 25.2 \mu \mathrm{m} / \mathrm{ns}$, in reasonable agreement with the Magboltz calculated $26.8 \mu \mathrm{m} / \mathrm{ns}$. In Chapter 6 , I made an approximate measurement of the longitudinal diffusion constant to be $D_{\mathrm{L}}(K E K) \approx 413 \mu \mathrm{m} / \sqrt{\mathrm{cm}}$ compared to the Magboltz value of $479 \mu \mathrm{m} / \sqrt{\mathrm{cm}}$, which shows the analysis is sensitive to longitudinal diffusion, but could be improved with the inclusion of charge dispersion effects in the pulse fitting algorithm.

The same set of measurements was made from an analysis of data collected in 2006 at the DESY facility in Germany. In this test, COSMo was set inside a $5 \mathrm{~T}$ superconducting magnetic, and used the so-called T2K gas mixture of: 95\% argon, $2 \%$ isobutane, and $3 \% \mathrm{CF}_{4}$, operating with a $200 \mathrm{~V} / \mathrm{cm}$ drift field. I measured the drift velocity to be $v_{\text {drift }}(D E S Y) \approx 74 \mu \mathrm{m} / \mathrm{ns}$, and the longitudinal diffusion constant $D_{\mathrm{L}}(D E S Y) \approx 259 \mu \mathrm{m} / \sqrt{\mathrm{cm}}$, both of which were found to be in reasonale agreement with Magboltz calculations.

Finally, the intrinsic longitudinal resolution was measured using the Geometric Mean method (described in Section 5.5). The resolution from the KEK test was found to be $\epsilon_{\mathrm{L}_{0}}(K E K) \approx 4.1 \mathrm{~ns}$, (equivalent to $\left.103 \mu \mathrm{m}\right)$. The DESY analysis yielded $\epsilon_{\mathrm{L}_{0}} \approx 7.2 \mu \mathrm{m}(535 \mu \mathrm{m})$. It is not surprising that the KEK resolution at zero drift distance is much better, given the much slower drift velocity. However, the cost is that the resolution is strongly dependent on drift distance, as can be seen in Figure 6.4, whereas the DESY resolution is relatively constant over the full TPC drift distance of $157 \mathrm{~mm}$. The T2K gas used in the DESY test is a much more likely candidate for future experiments due to the excellent transverse 
resolution that was achieved in a previous study (see Figure 4.6), so, that we see the longitudinal resolution close to the ILD requirement of $500 \mu \mathrm{m}$ is a promising result. An extrapolation to $2 \mathrm{~m}$ drift gives $\epsilon_{\mathrm{L}}=1240 \mu \mathrm{m}$, which is within the 1400 $\mu \mathrm{m}$ goal. I have also found that re-binning the pulses to larger time bin widths (refer to Tables 6.1 and 6.2) has little effect on the results, which is important because it is unlikely that a large scale TPC would use $200 \mathrm{MHz}$ FADCs due to the higher cost.

Some in the TPC community have questioned the use of charge dispersion because of the negative effect they believed it would have on timing resolution. Although it is difficult to make a true comparison of the performance of the COSMo TPC with other detectors, as there are so many variables involved, we can safely say that we are within the same ballpark. Table 7.1 contains the longitudinal resolution of other time projection chambers. The UVic UVTPC [10] and Carleton-GEM TPC [19] were prototypes of similar dimensions to COSMo, but used double GEM foils for amplification and had conventional (direct charge collection) readout pads. The COSMo results certainly appears better, however, it should be noted that the UVTPC experiment was not designed to measure the longitudinal resolution.

STAR [21] and ALEPH [7] were both full scale TPC's that used the conventional MWPC's for amplification, with drift time determined from signals on sense wires. With similar gas parameters and drift fields, both detectors achieved $\sim 750 \mu \mathrm{m}$ resolution for small $\theta$ tracks, over their full $\approx 2 \mathrm{~m}$ drift distances. One 


\begin{tabular}{|c|c|c|c|c|c|c|}
\hline \multirow[t]{2}{*}{ TPC } & \multirow[t]{2}{*}{ Amplifier } & \multirow[t]{2}{*}{ Readout } & \multirow[t]{2}{*}{ Gas } & \multirow{2}{*}{$\begin{array}{c}\mathbf{v}_{\mathrm{drrft}} \\
(\mu \mathrm{m} / \mathrm{ns})\end{array}$} & \multicolumn{2}{|c|}{$\epsilon_{\mathrm{L}_{0}}$} \\
\hline & & & & & (ns) & $(\mu \mathrm{m})$ \\
\hline \multirow[t]{2}{*}{ UVTPC } & \multirow[t]{2}{*}{ double GEM } & \multirow{2}{*}{$\begin{array}{l}\text { Anode } \\
\text { pads }\end{array}$} & $\mathrm{Ar}: \mathrm{CH}_{4}(\mathrm{P} 5)$ & 38.4 & 23.5 & 902 \\
\hline & & & $\mathrm{Ar}: \mathrm{CH}_{4}: \mathrm{CO}_{2}$ & 45.1 & 20.5 & 925 \\
\hline \multirow{2}{*}{$\begin{array}{l}\text { Carleton } \\
\text {-GEM }\end{array}$} & \multirow{2}{*}{ double GEM } & \multirow{2}{*}{$\begin{array}{l}\text { Anode } \\
\text { pads }\end{array}$} & $\mathrm{Ar}: \mathrm{CH}_{4}(\mathrm{P} 10)$ & 55 & 13 & 715 \\
\hline & & & $\mathrm{Ar}: \mathrm{CO}_{2}$ & 8.9 & 9 & 80 \\
\hline STAR & MWPC & $\begin{array}{l}\text { Wire/ } \\
\text { pads }\end{array}$ & $\mathrm{Ar}: \mathrm{CH}_{4}(\mathrm{P} 10)$ & 54.5 & 14 & $750^{*}$ \\
\hline ALEPH & MWPC & $\begin{array}{l}\text { Wire/ } \\
\text { pads }\end{array}$ & $\mathrm{Ar}: \mathrm{CH}_{4}(\mathrm{P} 9)$ & 52.4 & 14 & $740^{*}$ \\
\hline \multirow[t]{2}{*}{ COSMo } & \multirow[t]{2}{*}{ Micromegas } & \multirow{2}{*}{$\begin{array}{l}\text { Resistive } \\
\text { anode }\end{array}$} & $\mathrm{Ar}: \mathrm{C}_{4} \mathrm{H}_{10}$ & 25.5 & 4.08 & 103 \\
\hline & & & $\mathrm{Ar}: \mathrm{C}_{4} \mathrm{H}_{10}: \mathrm{CF}_{4}$ & 74 & 7.29 & 535 \\
\hline
\end{tabular}

TABLE 7.1: Comparison of COSMo TPC $\epsilon_{\mathrm{L}_{0}}$ with two other small prototype detectors: UVTPC [10], and Carleton-GEM TPC [19]; and two full scale detectors: STAR TPC [21] and ALEPH TPC [7]. The resolution listed for STAR and ALEPH is are approximately independent of $z$.

needs to consider the track segment size that these MWPC detectors had compared to the MPGD TPCs. For example, ALEPH had $30 \mathrm{~mm}$ long pads, compared to the $6 \mathrm{~mm}$ long COSMo pads, therefore each ALEPH time measurement would have $\sim 5 \times$ better electron statistics.

Although we have shown the ILD-TPC longitudinal resolution goals are within reach, there is still much room for improvement and future work to be done. The methods that I developed ignored the effect of charge dispersion on the shape of the primary pulse. Adding the charge signals from the neighbouring pads to the primary signal would account for most of the charge lost from the primary signal due to dispersion. I was unable to take advantage of this, however it should 
be further investigated with a more robust algorithm, possibly that includes fitting the entire pulse shape, rather than just the leading edge. One alternative may be to avoid the dispersed charge altogether by measuring the arrival time directly off the Micromegas mesh, which would be equivalent to summing all pads together, but without the addition of noise. 


\section{Appendix A}

\section{Justification of Linear Track Fit}

\section{for $(\mathbf{t}, \theta)$}

When a charged particle traverses a Time Projection Chamber (TPC), it is subject to the Lorentz force:

$$
\mathbf{F}=q(\mathbf{E}+\mathbf{v} \times \mathbf{B}) .
$$

With both electric and magnetic fields uniform and parallel to the cylindrical axis of the chamber, the force on the moving particle is decoupled into a force in the transverse plane $\mathrm{F}_{t}=q v_{t} \mathrm{~B}$ and a force in the longitudinal plane $\mathrm{F}_{l}=q \mathrm{E}$, as shown in Figure A.1. The magnetic field causes the particle trajectory to curve in the transverse plane of the fields, while the electric field causes the trajectory to curve in the longitudinal plane of the fields. 


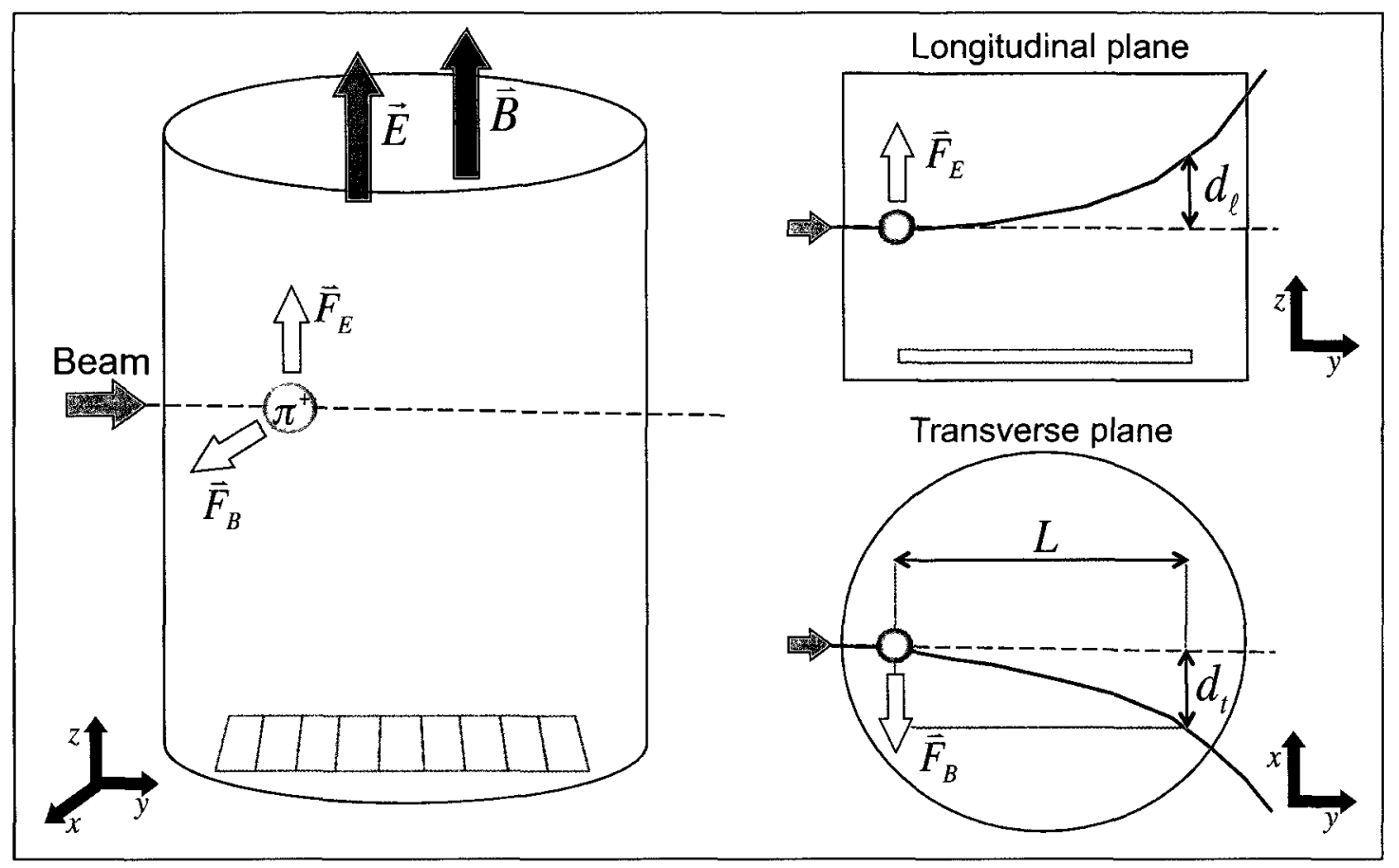

FIGURE A.1: The forces on a positively charged beam particle $\left(\pi^{+}\right)$inside a TPC. The track curvature in both the Longitudinal and Transverse planes is greatly exaggerated to show the displacements $d_{l}$ and $d_{t}$.

To show that the curvature in the longitudinal plane is sufficiently small to treat it as a straight line, we derive a simple equation for the maximum longitudinal deflection, $d_{l}$, of the particle trajectory away from the linear case (the result in the absence of the electric field). Since the force in the longitudinal direction is constant, the actual trajectory should be parabolic, and the deflection from the linear case is simply:

$$
d_{l}=\frac{1}{2} a t^{2}
$$

where $a$ is the acceleration of the particle, and $t$ is the total time spent in the TPC. Taking the particle to be a pion, travelling near the speed of light, $c$, the 
path length of the particle above the readout is $L_{\text {plane }}(7 \times 6 \mathrm{~mm}=4.2 \mathrm{~cm})$, and substituting in the longitudinal force $(q \mathrm{E})$, then the deflection becomes:

$$
d_{l}=\frac{q \mathrm{E}}{2 m_{\pi^{+}}}\left(\frac{L_{\text {plane }}}{c}\right)^{2},
$$

which, for the $\pi^{+}$at $\mathrm{KEK}\left(m_{\pi^{+}}=139.6 \mathrm{MeV} / \mathrm{c}^{2}\right)$, is approximately $44 \mathrm{~nm}$. To convert the deflection distance into a difference in arrival times of electron clusters, we use the drift velocity, $(25.2 \mu \mathrm{m} / \mathrm{ns})$ giving $t_{l}=0.0017 \mathrm{~ns}$. To put this in perspective, our best timing resolution measured from the KEK experiment was $\sim 4$ ns, over 2000 times greater.

Repeating this calculation for a cosmic $\mu^{-}\left(m_{\mu^{-}}=105.7 \mathrm{MeV} / \mathrm{c}^{2}\right)$ at the DESY experiment $(E=200 \mathrm{~V} / \mathrm{cm})$, gives $d_{l}=166 \mathrm{~nm}$. Using the drift velocity $(74 \mu / \mathrm{ns})$ to convert to time gives $t_{l}=0.0022 \mathrm{~ns}$, which again is three orders of magnitude smaller than our best resolution measurement. Therefore the approximation we have made using a linear track fit in $(z(t), y)$ appears to be a safe one. 
Appendix B

\section{Additional Figures}




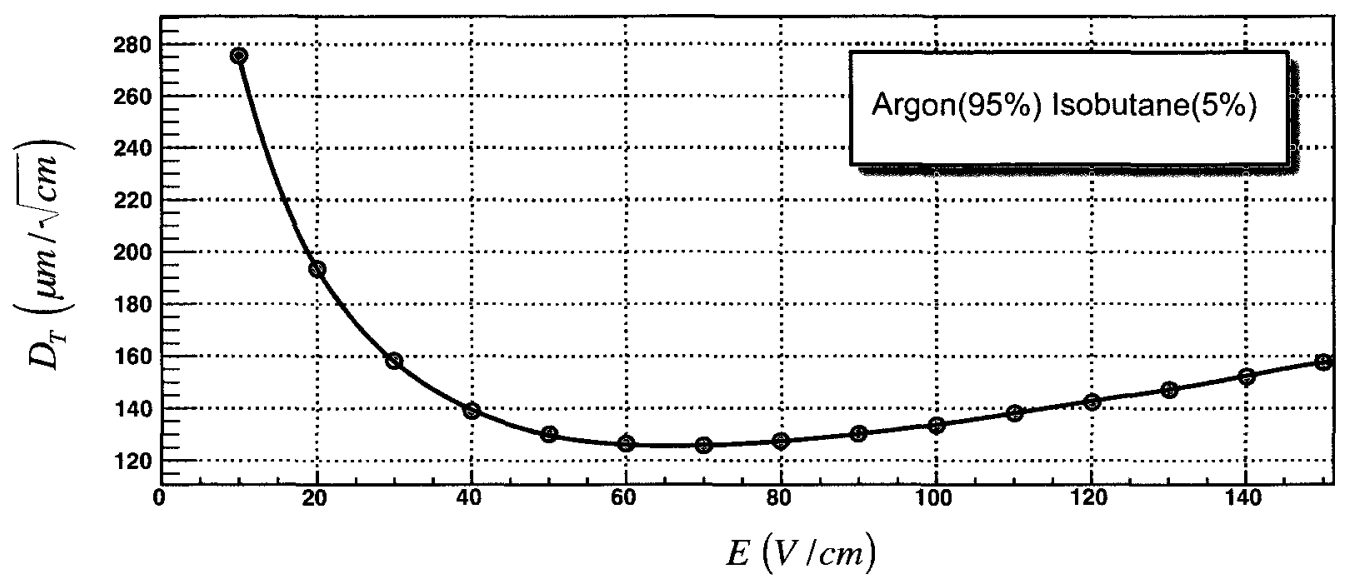

(a) Transverse Diffusion

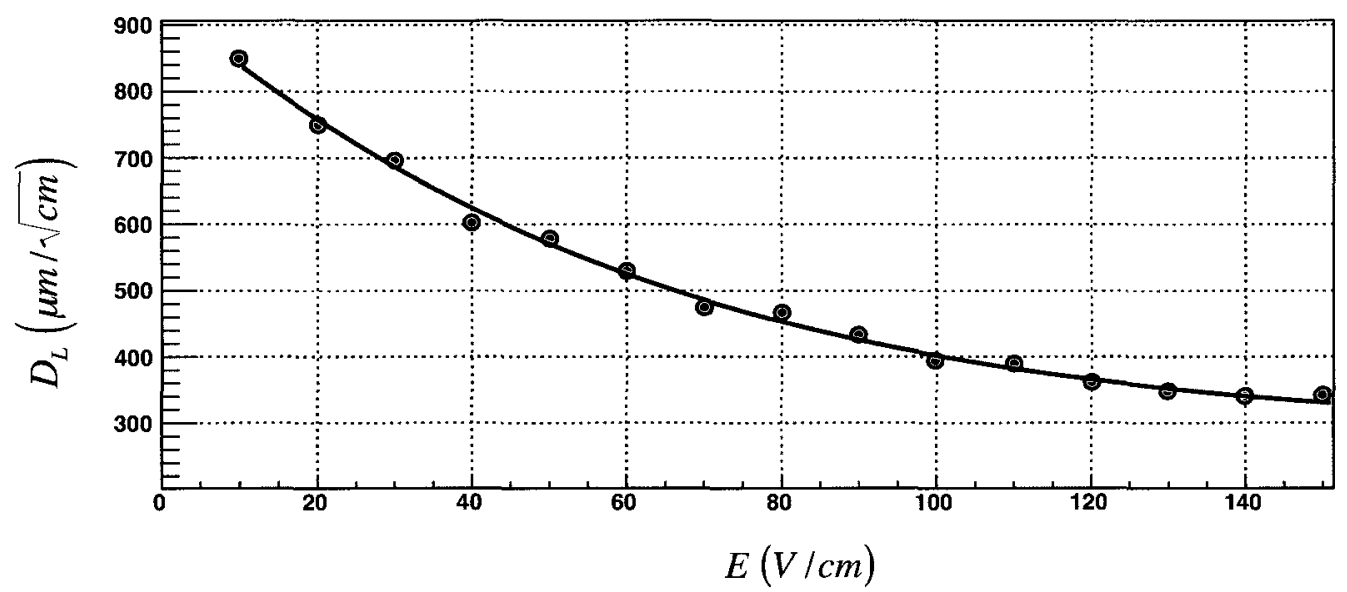

(b) Longitudinal Diffusion

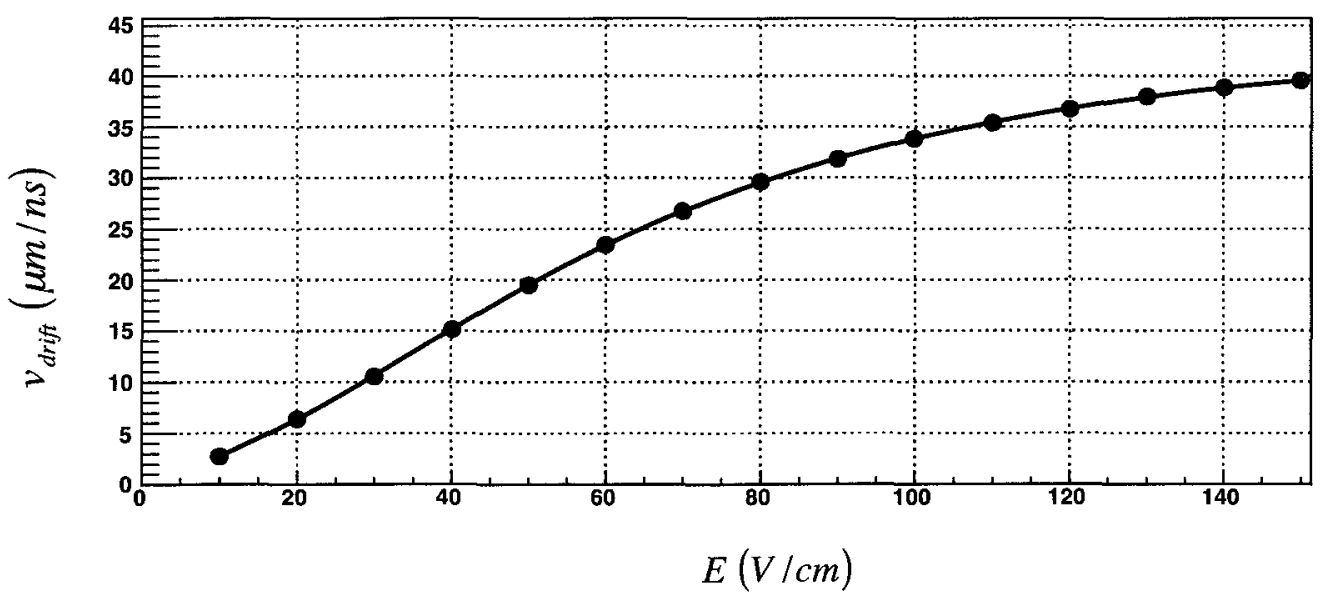

(c) Drift Velocity

Figure B.1: MAGBOLTZ calculations for (a) Transverse Diffusion, (b) Longitudinal Diffusion, and (c) Drift Velocity, as functions of drift field for the gas mixture used in the KEK 2005 experiment (Argon-Isobutane), with $B=1 \mathrm{~T}$. 


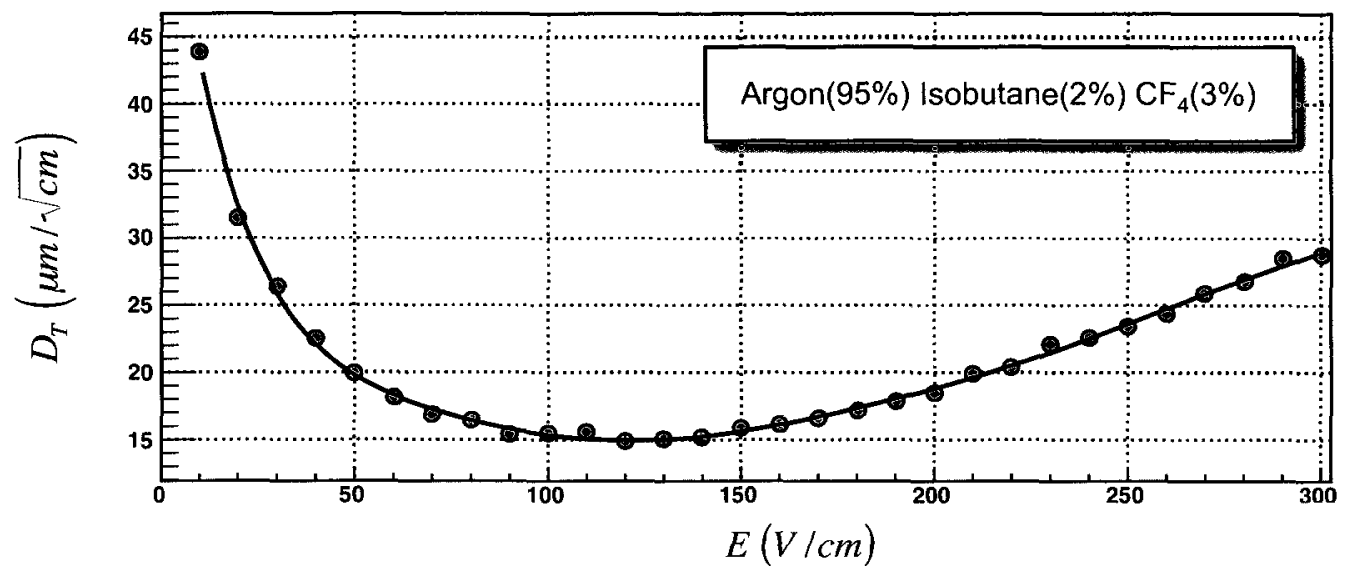

(a) Transverse Diffusion

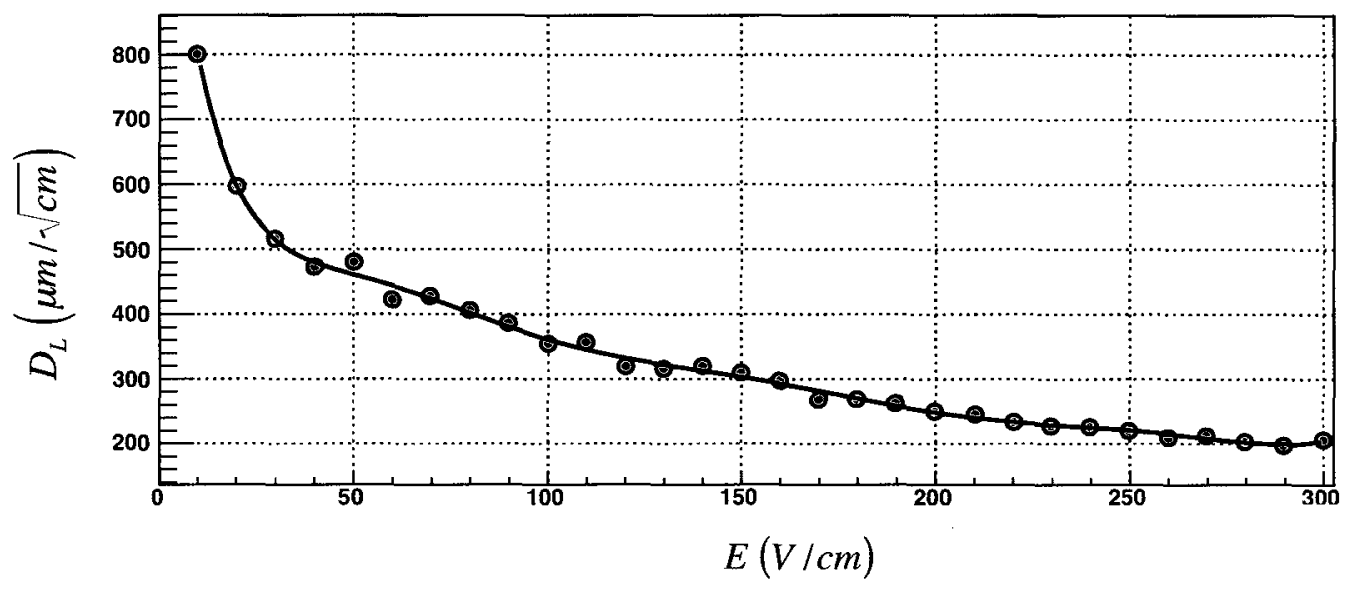

(b) Longitudinal Diffusion

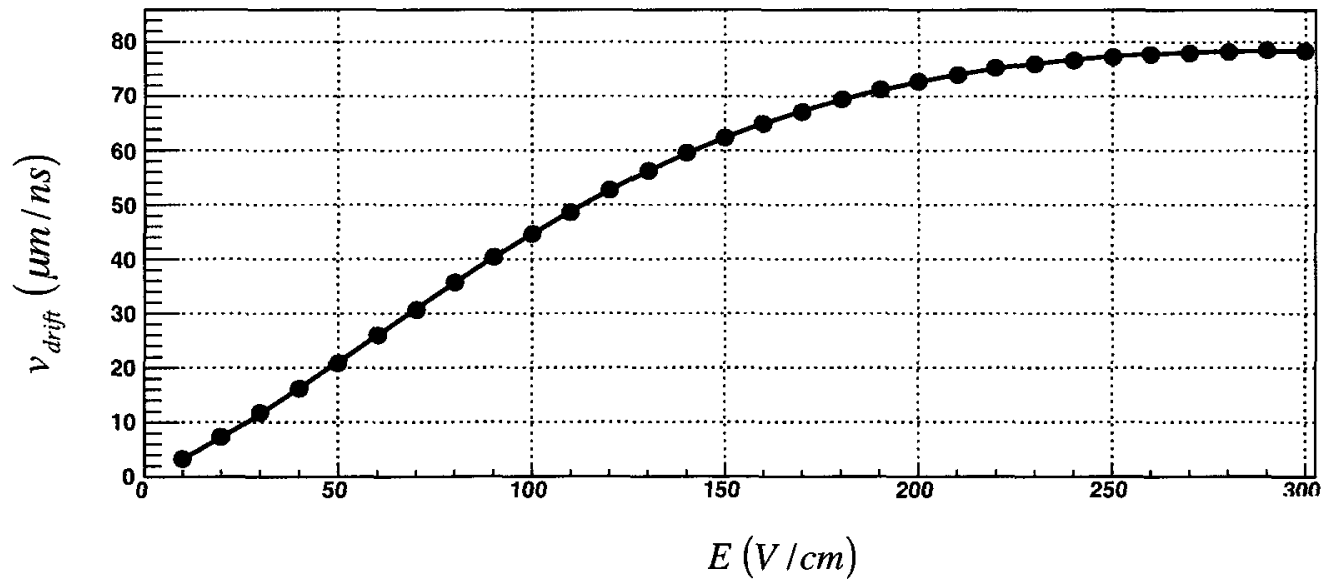

(c) Drift Velocity

FIgURE B.2: MAGBOLTZ calculations for (a) Transverse Diffusion, (b) Longitudinal Diffusion, and (c) Drift Velocity, as functions of applied drift field for the gas mixture used in the DESY 2006 experiment (Argon-Isobutane- $\mathrm{CF}_{4}$ ) with $B=5 \mathrm{~T}$. 


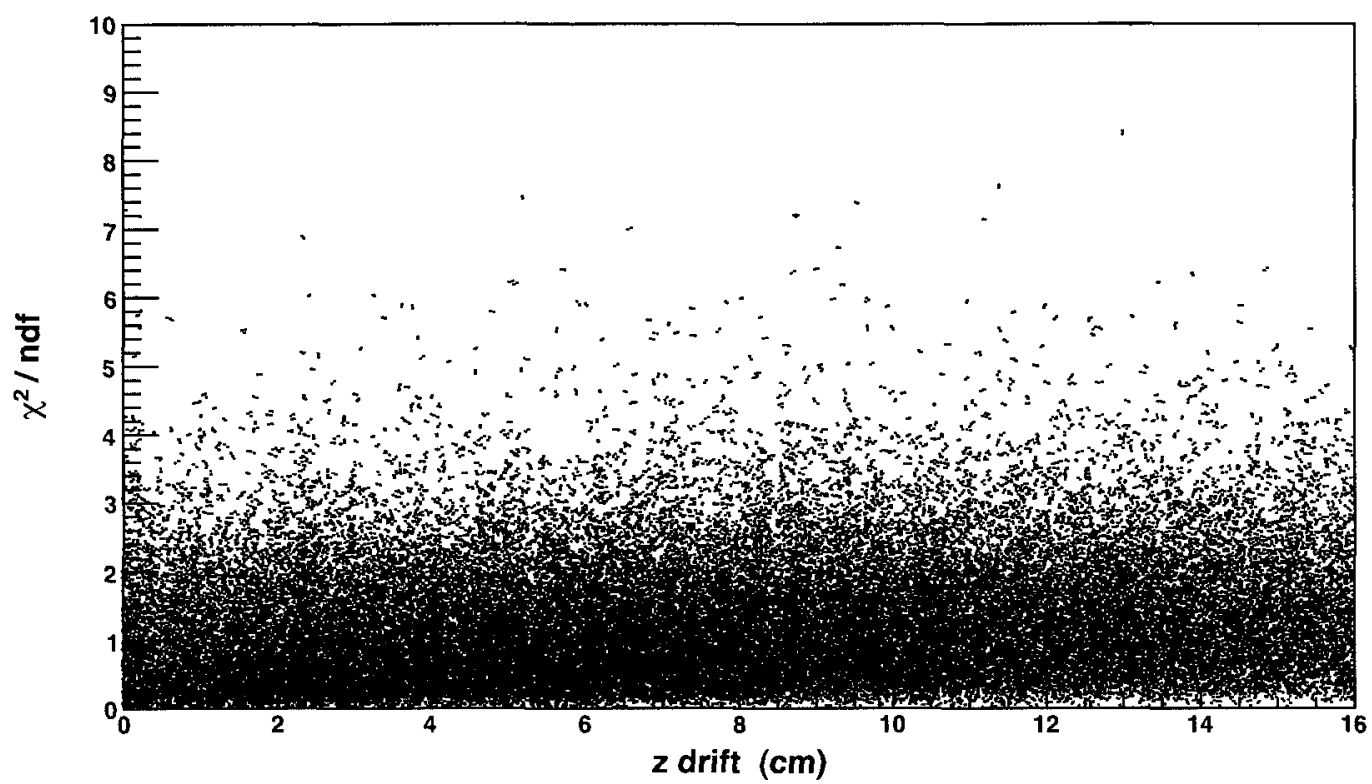

Figure B.3: KEK pulse fit results: $\chi^{2} / n d f$ vs drift distance. (Error function Fit Method, 25 ns time bins)

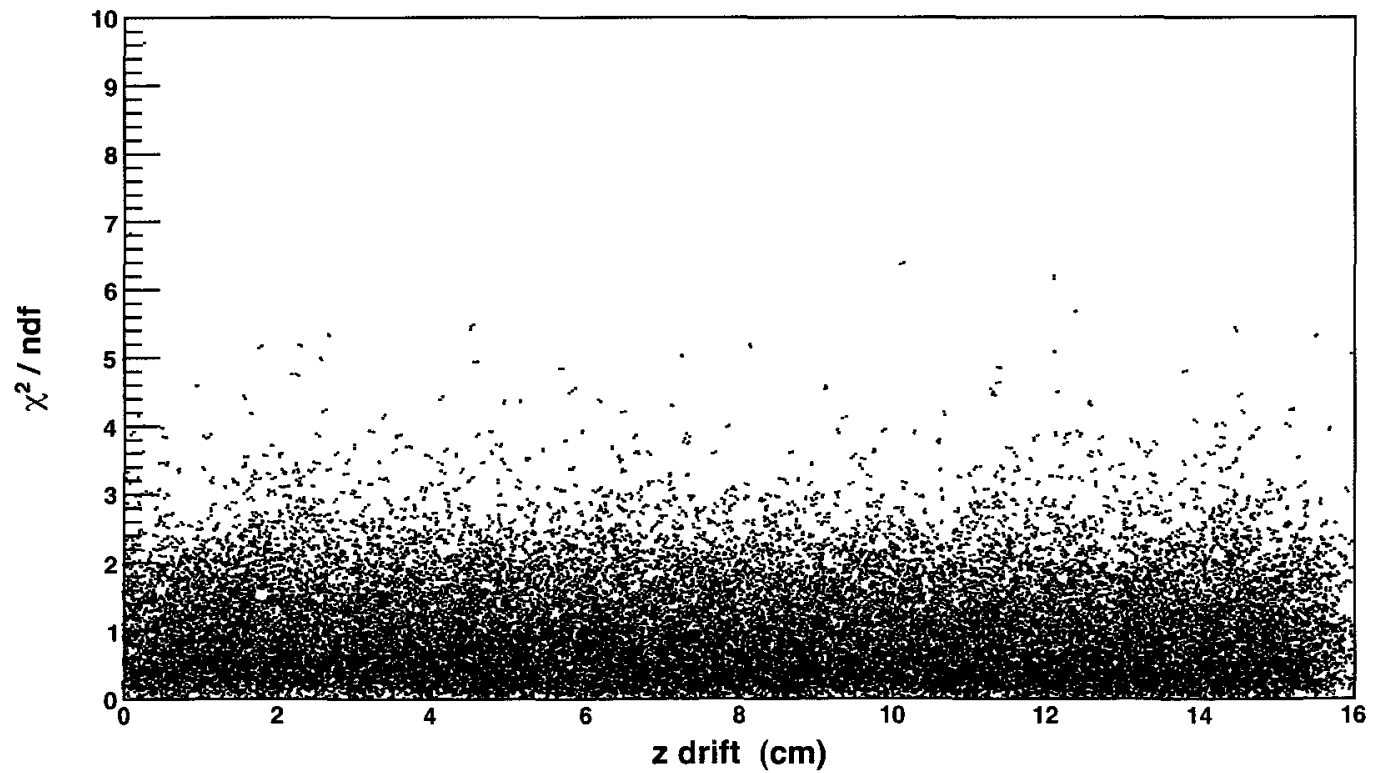

FiguRE B 4: DESY pulse fit results: $\chi^{2} / n d f$ vs drift distance. (Error function Fit Method, 25 ns time bins) 


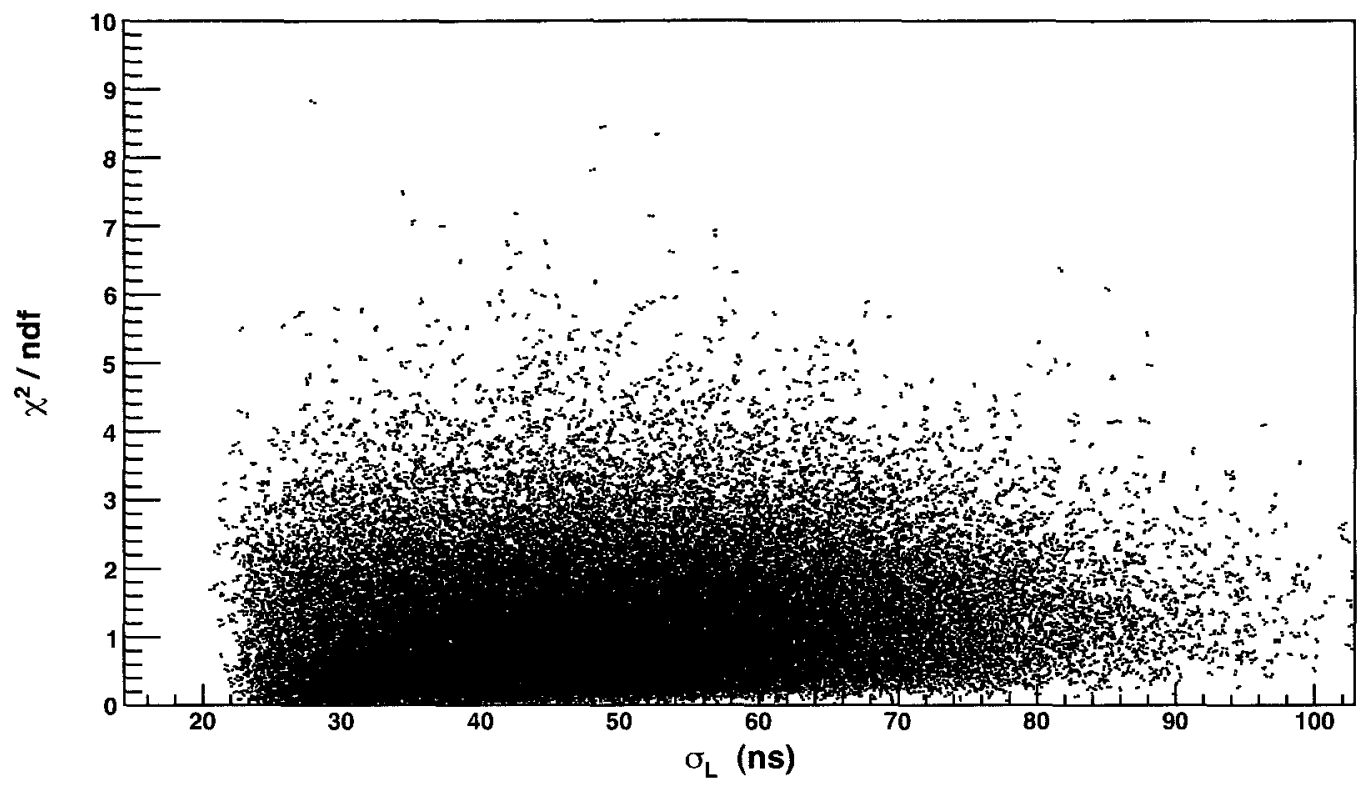

Figure B.5: KEK pulse fit results: $\chi^{2} / n d f$ vs $\sigma_{L}$ (pulse width). (Error function Fit Method, 25 ns time bins)

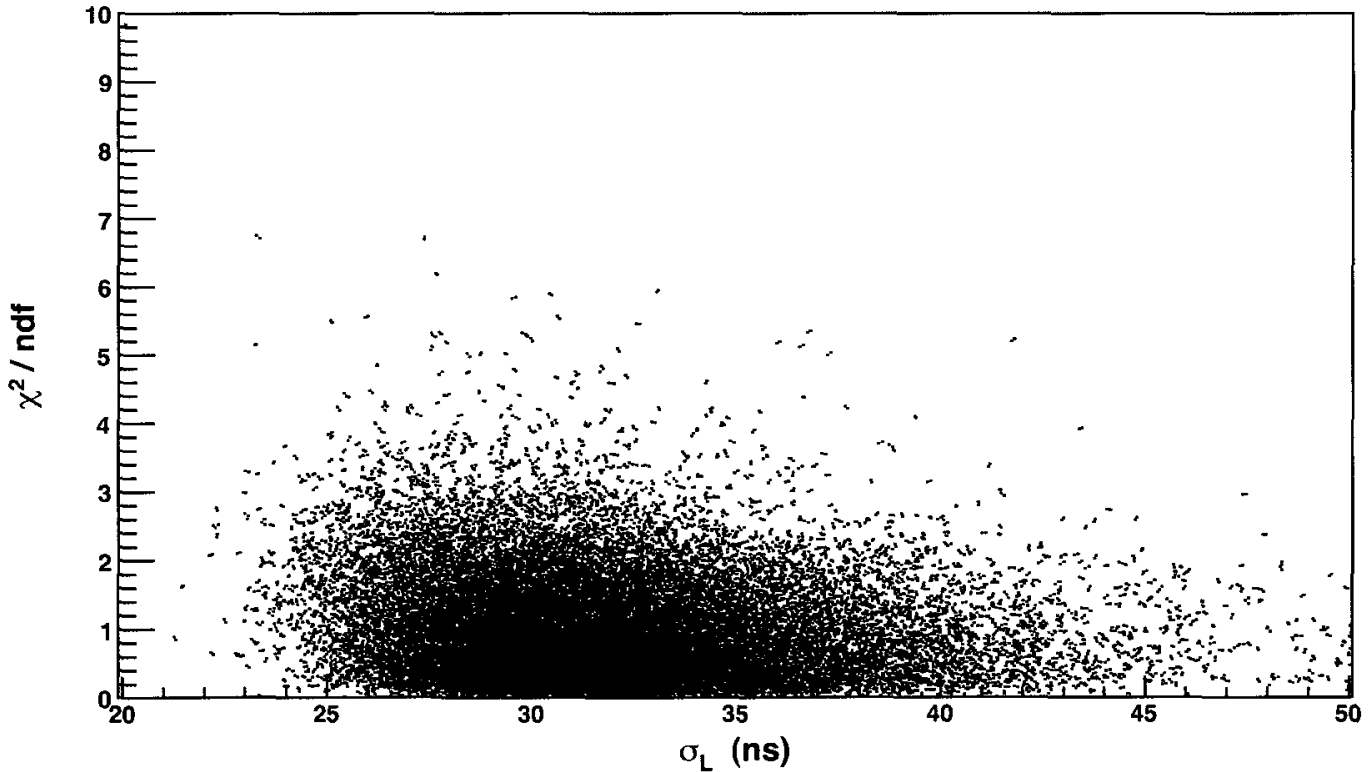

Figure B.6. DESY pulse fit results: $\chi^{2} / n d f$ vs $\sigma_{L}$ (pulse width). (Error function Fit Method, 25 ns time bins) 

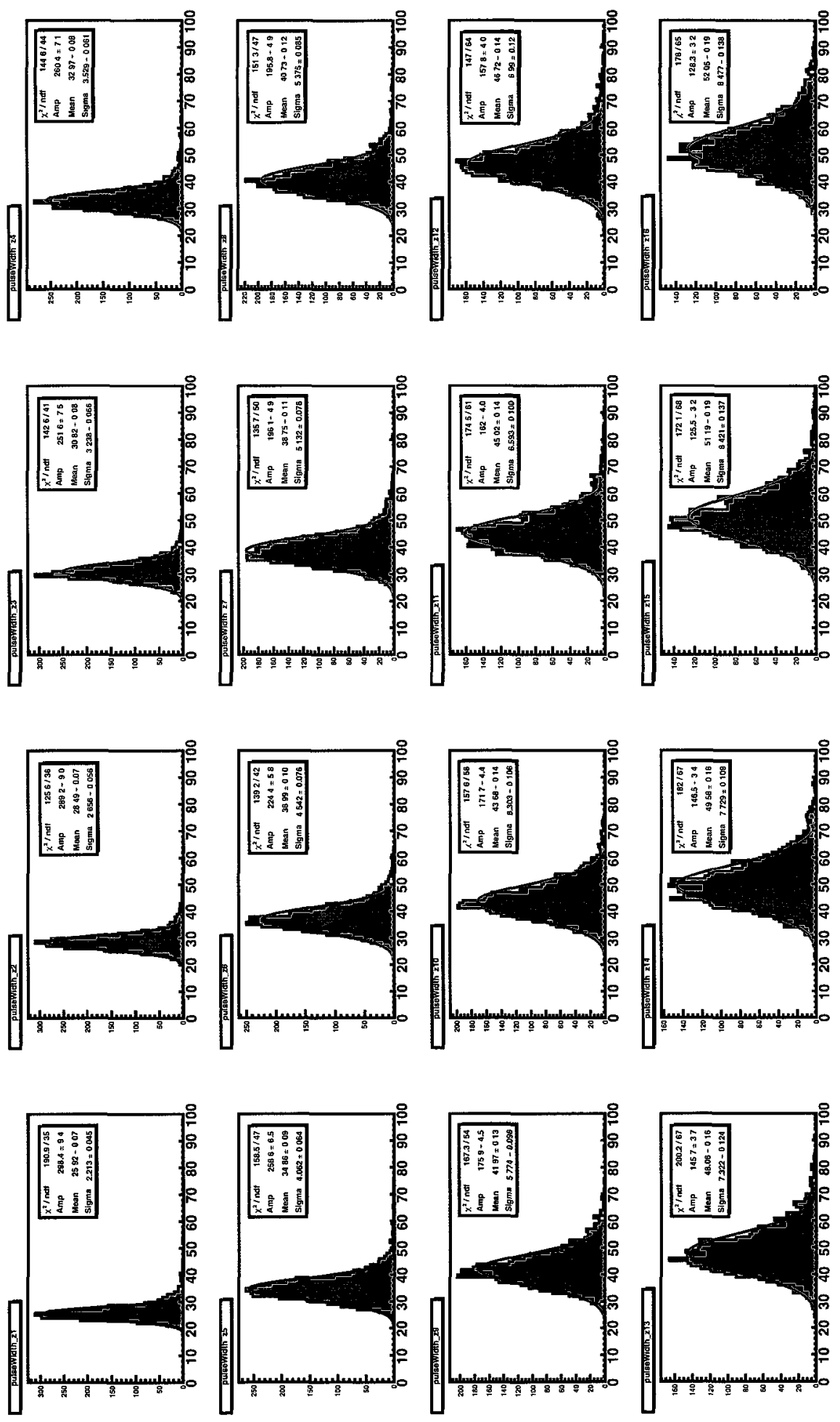

FIGURE B.7: KEK pulse width $\left(\sigma_{L}\right)$ distributions, for drift distances $z \leq 8 \mathrm{~cm}$, in $0.5 \mathrm{~cm}$ wide bins. The Gaussian fitted mean of each distribution is plotted to determine the diffusion constant, $D_{\mathrm{L}}$. (Error function Fit Method, 25 ns time bins) 

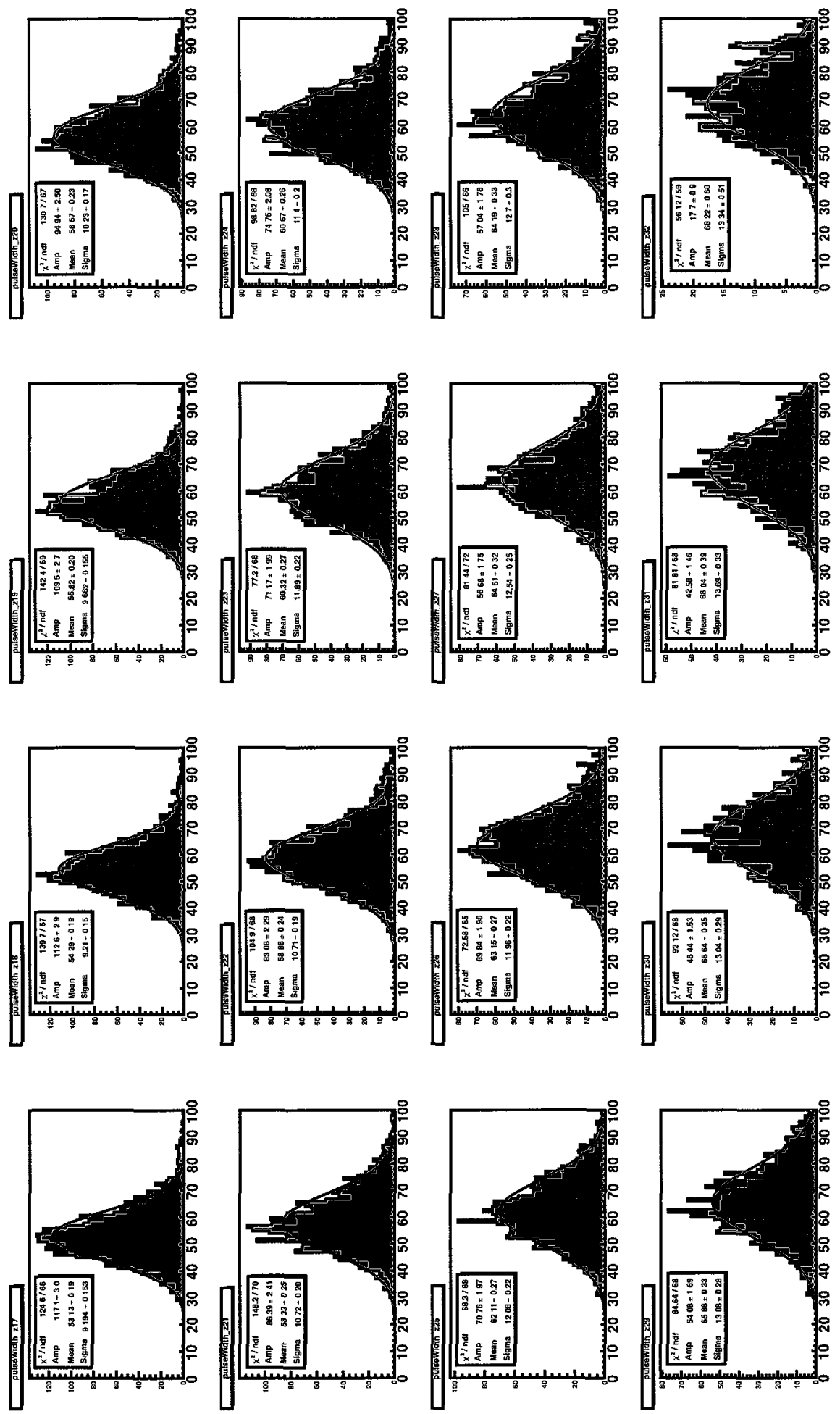

FigURE B.8: KEK pulse width $\left(\sigma_{L}\right)$ distributions, for drift distances $z>8 \mathrm{~cm}$, in $0.5 \mathrm{~cm}$ wide bins. The Gaussian fitted mean of each distribution is plotted to determine the diffusion constant, $D_{\mathrm{L}}$. (Error function Fit Method, 25 ns time bins) 

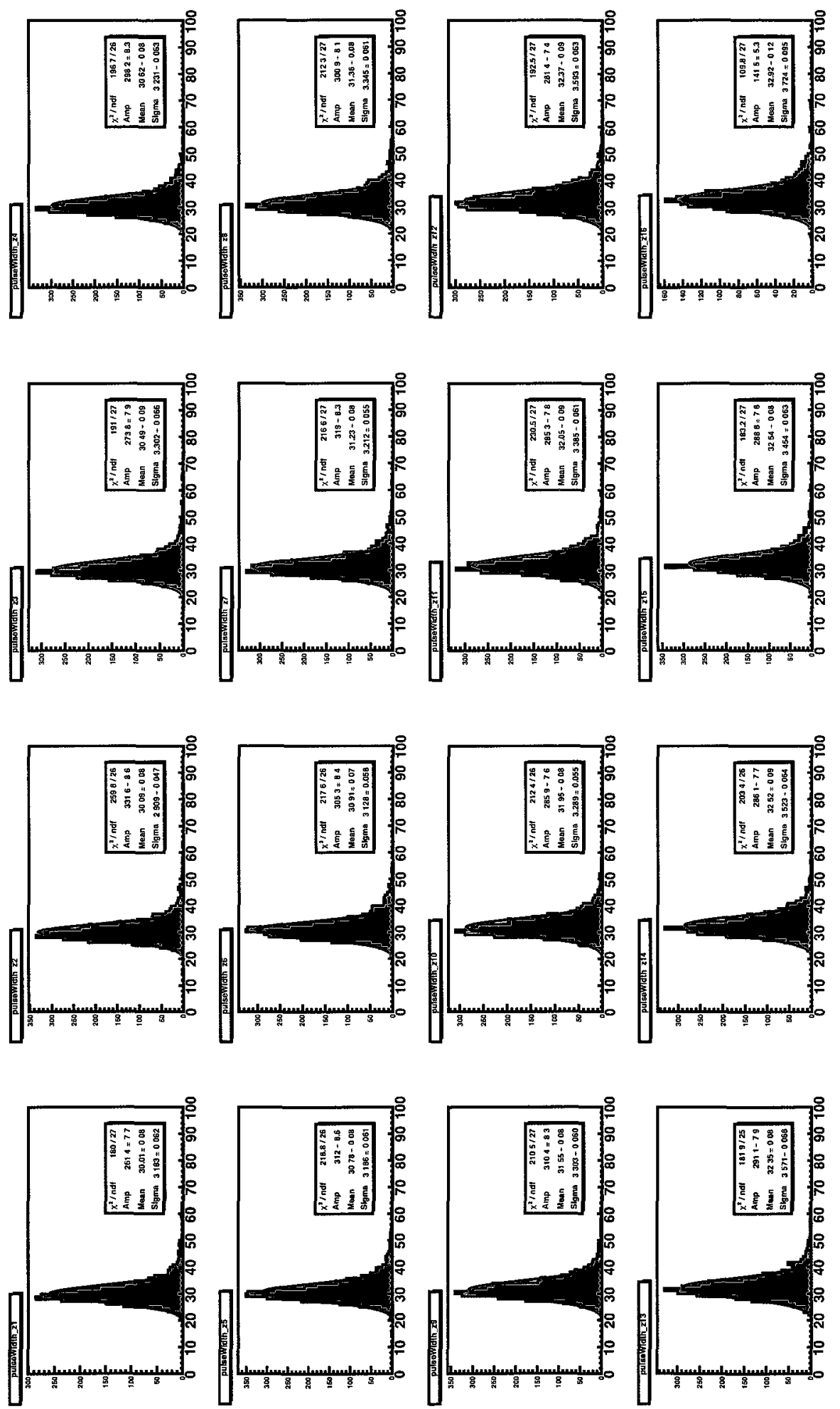

FiguRE B.9: DESY pulse width $\left(\sigma_{L}\right)$ distributions, for drift distances of 0.5 through $15.5 \mathrm{~cm}$, in $1 \mathrm{~cm}$ wide bins. The Gaussian fitted mean of each distribution is plotted to determine the diffusion constant, $D_{\mathrm{L}}$. (Error function Fit Method, 25 ns time bins) 


\section{Appendix $\mathrm{C}$}

\section{Numerical Differentiation}

The derivative of a function $f(x)$ at a point $x_{0}$, denoted $f^{\prime}\left(x_{0}\right)$, is defined by choosing a small change in $x$, denoted $h$. The derivative is then given by Newton's difference quotient [23], which is taken as the limit of the slope as $\mathrm{h}$ approaches zero:

$$
f^{\prime}\left(x_{0}\right)=\lim _{h \rightarrow 0} \frac{f\left(x_{0}+h\right)-f\left(x_{0}\right)}{h} .
$$

In the absence of the functional form of $f(x)$, the numerical derivative can thus be approximated as:

$$
f^{\prime}\left(x_{0}\right) \approx \frac{f\left(x_{0}+h\right)-f\left(x_{0}\right)}{h}
$$


where $h$ is now the bin width of our pulse data (ns). This is known as the forward difference approximation. Equally, we could choose $h$ to be negative, giving:

$$
f^{\prime}\left(x_{0}\right) \approx \frac{f\left(x_{0}\right)-f\left(x_{0}-h\right)}{h},
$$

which is the backward difference formula. Clearly the accuracy of these approximations must depend on the magnitude of $h$. We can check this by a Taylor expansion of the function $f(x)$ about the point $x_{0} \pm h$, and assuming the existence of higher order derivatives, we obtain:

$$
\begin{aligned}
& f\left(x_{0}+h\right)=f\left(x_{0}\right)+f^{\prime}\left(x_{0}\right) h+\frac{f^{\prime \prime}\left(x_{0}\right)}{2} h^{2}+\frac{f^{\prime \prime \prime}\left(\xi_{+}\right)}{6} h^{3}+\ldots \\
& f\left(x_{0}-h\right)=f\left(x_{0}\right)-f^{\prime}\left(x_{0}\right) h+\frac{f^{\prime \prime}\left(x_{0}\right)}{2} h^{2}-\frac{f^{\prime \prime \prime}\left(\xi_{-}\right)}{6} h^{3}+\ldots
\end{aligned}
$$

Keeping terms to second order and solving for $f^{\prime}\left(x_{0}\right)$ yields:

$$
f^{\prime}\left(x_{0}\right)=\frac{ \pm f\left(x_{0} \pm h\right) \mp f\left(x_{0}\right)}{h}+\frac{f^{\prime \prime}\left(\xi_{ \pm}\right)}{2} h,
$$

where $\xi_{+} \in[x 0, x 0+h]$ and $\xi_{-} \in\left[x_{0}-h, x_{0}\right]$. So the forward and backward difference approximations are only accurate to first order in $h$. To improve upon this, we combine the two by taking the average:

$$
f^{\prime}\left(x_{0}\right)=\frac{f\left(x_{0}+h\right)-f\left(x_{0}-h\right)}{2 h},
$$


which is known as the centred difference approximation. To determine the accuracy of this approximation, we again use the Taylor expansions as above, keeping terms to third order. By subtracting equation C.5 from C.4, the second order terms cancel out. Solving for $f^{\prime}\left(x_{0}\right)$ yields:

$$
f^{\prime}\left(x_{0}\right)=\frac{f\left(x_{0}-h\right)-f\left(x_{0}+h\right)}{2 h}-\frac{f^{\prime \prime \prime}\left(\xi_{+}\right)+f^{\prime \prime \prime}\left(\xi_{-}\right)}{12} h^{2}
$$

which can be simplified by invoking the Intermediate Value Theorem to:

$$
f^{\prime}\left(x_{0}\right)=\frac{f\left(x_{0}-h\right)-f\left(x_{0}+h\right)}{2 h}-\frac{f^{\prime \prime \prime}(\xi)}{6} h^{2},
$$

where $\xi \in\left[x_{0}-h, x_{0}+h\right]$. So the centred difference approximation is accurate to second order, an improvement!

Higher order difference approximations can be found using the same Taylor expansion process, however this becomes tedious as the number of simultaneous equations increases. The same results can be achieved by a different approach of differentiating Lagrange interpolating polynomials [24, 25]. The Lagrange polynomials are defined as:

$$
\mathcal{L}_{\jmath}(\alpha)=\prod_{\imath=1, \imath \neq \jmath}^{k} \frac{\alpha-x_{\imath}}{x_{\jmath}-x_{\imath}}
$$

To derive a 5 -point difference approximation, we choose the $k=5$ interpolation points: $x_{1}\left(=x_{0}-2 h\right), x_{2}\left(=x_{0}-h\right), x_{3}\left(=x_{0}\right), x_{4}\left(=x_{0}+h\right), x_{5}\left(=x_{0}+2 h\right)$. The 
resulting fourth-degree polynomial can then be expressed as:

$$
p_{4}(x)=\sum_{\jmath=1}^{5} f\left(x_{\jmath}\right) \mathcal{L}_{\jmath}(x)
$$

where $f\left(x_{\jmath}\right)$ are the interpolation point values. The derivative of the polynomial is then:

$$
p_{4}^{\prime}(x)=\sum_{\jmath=1}^{5} f\left(x_{\jmath}\right) \mathcal{L}_{\jmath}^{\prime}(x)
$$

Writing out the terms, the 5-point difference approximation of $f^{\prime}(x)$ at the centre point $x=x_{0}$ is:

$$
\begin{aligned}
f^{\prime}\left(x_{0}\right) \approx p_{4}^{\prime}\left(x_{0}\right)=f\left(x_{1}\right) \mathcal{L}_{1}^{\prime}\left(x_{0}\right) & +f\left(x_{2}\right) \mathcal{L}_{2}^{\prime}\left(x_{0}\right)+f\left(x_{3}\right) \mathcal{L}_{3}^{\prime}\left(x_{0}\right) \\
& +f\left(x_{4}\right) \mathcal{L}_{4}^{\prime}\left(x_{0}\right)+f\left(x_{5}\right) \mathcal{L}_{5}^{\prime}\left(x_{0}\right)
\end{aligned}
$$

Expanding these terms and simplifying is quite labour intensive by hand, but straight forward for a program such as Maple, and gives the expected form:

$$
f^{\prime}\left(x_{0}\right) \approx \frac{f\left(x_{0}-2 h\right)-8 f\left(x_{0}-h\right)+8 f\left(x_{0}+h\right)-f\left(x_{0}+2 h\right)}{12 h},
$$

which is accurate to fourth order (shown by Taylor expanding the right hand side). The same recipe can be followed for higher ordered approximations, however this is sufficient, given the limited number of points on the leading edge of a charge pulse. 


\section{Appendix D}

\section{Weighted Least-Squares Fit}

The derivation of the weighted least-squares fit to a straight line can be found in [22], and proceeds as follows. Suppose that we have $N$ independent measurements of time, $t_{2}$, corresponding to known pad row positions, $y_{2}$. Assuming random errors, the $t_{\imath}$ will be Gaussian distributed with width $\sigma_{\imath}$ about the most probable value $\left\langle t_{\imath}\right\rangle$, where:

$$
<t_{\imath}>=A+B y_{\imath},
$$

where $A$ is the $t$-intercept and $B$ is the slope of the track. Given these track parameters $\mathrm{A}$ and $\mathrm{B}$, the probability of measuring a given $t_{\imath}$ is:

$$
P\left(t_{\imath}\right)_{A, B} \propto \frac{1}{\sigma_{t_{\imath}}} \exp \left[-\frac{1}{2}\left(\frac{t_{\imath}-\left(A+B y_{\imath}\right)}{\sigma_{t_{\imath}}}\right)^{2}\right] .
$$


Therefore, the probability of all $N$ measurements $\left(t_{1}, t_{2}, \ldots t_{N}\right)$ together is:

$$
\begin{aligned}
P\left(t_{1}, t_{2}, \ldots, t_{N}\right)_{A, B} & =P\left(t_{1}\right)_{A, B} \times P\left(t_{2}\right)_{A, B} \times \ldots \times P\left(t_{N}\right)_{A, B} \\
& \propto \exp \left(-\chi^{2} / 2\right) \prod_{\imath=1}^{N} \frac{1}{\sigma_{t_{2}}}
\end{aligned}
$$

where

$$
\chi^{2}=\sum_{\imath=1}^{N}\left(\frac{t_{\imath}-\left(A+B y_{\imath}\right)}{\sigma_{t_{\imath}}}\right)^{2} .
$$

The best estimate of $\mathrm{A}$ and $\mathrm{B}$ would occur if each $t_{\imath}=\left\langle t_{\imath}\right\rangle$, which must also be the most probable set of measurements. Therefore, we want to maximize D.3, which is equivalent to minimizing the value of $\chi^{2}$. This leads to two simultaneous equations for the fit parameters:

$$
\begin{aligned}
& \frac{\partial \chi^{2}}{\partial A}=-2 \sum_{\imath=1}^{N} \frac{1}{\sigma_{t_{\imath}}^{2}}\left(t_{\imath}-A-B y_{\imath}\right)=0, \\
& \frac{\partial \chi^{2}}{\partial B}=-2 \sum_{\imath=1}^{N} \frac{y_{\imath}}{\sigma_{t_{\imath}}^{2}}\left(t_{\imath}-A-B y_{\imath}\right)=0 .
\end{aligned}
$$

We define the weight factor to be $w_{\imath}=1 / \sigma_{t_{2}}^{2}$. Solving for the constants $A$ and $B$ we find:

$$
A=\frac{\sum_{\imath=1}^{N} w_{\imath} y_{\imath}^{2} \sum_{\imath=1}^{N} w_{\imath} t_{\imath}-\sum_{\imath=1}^{N} w_{\imath} y_{\imath} \sum_{\imath=1}^{N} w_{\imath} y_{\imath} t_{\imath}}{\sum_{\imath=1}^{N} w_{\imath} \sum_{\imath=1}^{N} w_{\imath} y_{\imath}^{2}-\left(\sum_{\imath=1}^{N} w_{\imath} y_{\imath}\right)^{2}},
$$




$$
B=\frac{\sum_{\imath=1}^{N} w_{\imath} \sum_{\imath=1}^{N} w_{\imath} y_{\imath} t_{\imath}-\sum_{\imath=1}^{N} w_{\imath} y_{\imath} \sum_{\imath=1}^{N} w_{\imath} t_{\imath}}{\sum_{\imath=1}^{N} w_{\imath} \sum_{\imath=1}^{N} w_{\imath} y_{\imath}-\left(\sum_{\imath=1}^{N} w_{\imath} y_{\imath}\right)^{2}} .
$$

In this case, the solution to the problem of finding the equation parameters which best fit the data points has an analytical solution. In general, this is not the case, and an algorithm (such as the MINUIT class used by ROOT), must be used to determine the parameters corresponding to a minimum $\chi^{2}$. 


\section{Appendix E}

\section{Geometric Mean Method}

The derivation of the Geometric Mean Method for calculating single-hit resolution, found in [19], is as follows. Supposing that we have a straight track consisting of $\mathrm{N}$ independent time measurements, $t_{\jmath}$, at known pad row positions $y_{\jmath}$, where $1 \leq \jmath \leq N$. Each time measurement should be randomly distributed about the expected mean $\left\langle t_{\jmath}\right\rangle$ with standard deviation $\epsilon_{\jmath}$. Then the true track position is:

$$
<t_{\jmath}>=A+B y_{\jmath}
$$

with $t$-intercept $A$, and slope $B$. Since a TPC provides just one measurement $t_{j}$ for each $y_{\jmath}$, we cannot calculate $\epsilon_{\jmath}$ directly (the point resolution). However, we can estimate the resolution of a single measurement, $i$. To simplify the calculation, the coordinates are chosen such that $y_{\imath}=0$, and therefore the residual is $R_{\imath}=A-t_{\imath}$ where the track parameter $A$ can be estimated using the weighted least-squares 
fit equation, either including $\left(A^{I N C}\right)$ or excluding $\left(A^{E X C}\right)$ the $t_{\imath}$ measurement:

$$
A^{I N C}=\frac{\sum_{k} w_{k} y_{k}^{2} \sum_{\jmath} w_{\jmath} t_{\jmath}-\sum_{k} w_{k} y_{k} \sum_{\jmath} w_{\jmath} y_{\jmath} t_{\jmath}}{\Delta^{I N C}},
$$

where

$$
\Delta^{I N C}=\sum_{\jmath} w_{\jmath} \sum_{\jmath} w_{\jmath} y_{\jmath}^{2}-\left(\sum_{\jmath} w_{\jmath} y_{\jmath}\right)^{2}
$$

and:

$$
A_{\imath}^{E X C}=\frac{\sum_{k \neq \imath} w_{k} y_{k}^{2} \sum_{\jmath \neq \imath} w_{\jmath} t_{\jmath}-\sum_{k \neq \imath} w_{k} y_{k} \sum_{\jmath \neq \imath} w_{\jmath} y_{\jmath} t_{\jmath}}{\Delta_{\imath}^{E X C}}
$$

where

$$
\Delta_{\imath}^{E X C}=\sum_{\jmath \neq \imath} w_{\jmath} \sum_{\jmath \neq \imath} w_{\jmath} y_{\jmath}^{2}-\left(\sum_{\jmath \neq \imath} w_{\jmath} y_{\jmath}\right)^{2}
$$

The inclusive and exclusive residuals will be distributed with standard deviations $\sigma_{I N C_{2}}$ and $\sigma_{E X C_{2}}$, which are related to $\epsilon_{\imath}$, but depend on the other measured points and their werght, defined as $w_{\jmath}=1 / \epsilon_{\jmath}^{2}$. Since we chose the coordinates such that $y_{\jmath=\imath}=0$, we also have the useful identity:

$$
\Delta_{\imath}^{E X C}=\Delta^{I N C}-w_{\imath} \sum_{\jmath} w_{\jmath} y_{\jmath}^{2}
$$


Now we can calculate the inclusive residual, $R_{\imath}^{I N C}$, of the $\imath^{\text {th }}$ point:

$$
\begin{aligned}
R_{\imath}^{I N C}= & A^{I N C}-t_{\imath} \\
= & \frac{\sum_{\jmath} w_{\jmath} t_{\jmath}\left(\sum_{k} w_{k} y_{k}^{2}-y_{\jmath} \sum_{k} w_{k} y_{k}\right)-t_{\imath} \Delta^{I N C}}{\Delta^{I N C}} \\
= & \frac{\sum_{\jmath} w_{\jmath} t_{\jmath}\left(\sum_{k} w_{k} y_{k}^{2}-y_{\jmath} \sum_{k} w_{k} y_{k}\right)-t_{\imath}\left(\Delta_{\imath}^{E X C}+w_{\imath} \sum_{\jmath} w_{\jmath} y_{\jmath}^{2}\right)}{\Delta^{I N C}} \\
= & \frac{\sum_{\jmath \neq \imath} w_{\jmath} t_{\jmath}\left(\sum_{k} w_{k} y_{k}^{2}-y_{\jmath} \sum_{k} w_{k} y_{k}\right)-t_{\imath} \Delta_{\imath}^{E X C}}{\Delta^{I N C}} .
\end{aligned}
$$

Since the $\mathrm{N}$ measurements are independent, the variance of the inclusive residual can be approxımated using error propagation as:

$$
\begin{aligned}
\sigma_{I N C_{\imath}}^{2} & =\left(\frac{\partial}{\partial t_{1}} R_{\imath}^{I N C} \epsilon_{1}\right)^{2}+\left(\frac{\partial}{\partial t_{2}} R_{\imath}^{I N C} \epsilon_{2}\right)^{2}+\cdots\left(\frac{\partial}{\partial t_{N}} R_{\imath}^{I N C} \epsilon_{N}\right)^{2} \\
& =\sum_{\jmath}\left(\frac{\partial}{\partial t_{\jmath}} R_{\imath}^{I N C} \epsilon_{\jmath}\right)^{2} .
\end{aligned}
$$

The partial differentiation picks out the $t_{\jmath}$ terms from $R_{\imath}^{I N C}$, yielding:

$$
\sigma_{I N C_{\imath}}^{2}=\frac{\sum_{j \neq \imath} w_{\jmath}^{2} \epsilon_{\jmath}^{2}\left(\sum_{k} w_{k} y_{k}^{2}-y_{\jmath} \sum_{k} w_{k} y_{k}\right)^{2}-\left(\Delta_{\imath}^{E X C} \epsilon_{\imath}\right)^{2}}{\left(\Delta^{I N C}\right)^{2}} .
$$

Expanding and collecting like terms yields:

$$
\sigma_{I N C_{\imath}}^{2}=\epsilon_{\imath}^{2} \frac{\Delta_{\imath}^{E X C}}{\Delta^{I N C}} .
$$


Repeating this for the exclusive residual variance, $\sigma_{E X C_{2}}^{2}$, gives the similar result:

$$
\sigma_{E X C_{\imath}}^{2}=\epsilon_{\imath}^{2} \frac{\Delta^{I N C}}{\Delta_{\imath}^{E X C}} .
$$

Combining E.10 and E.11 gives:

$$
\epsilon_{\imath}=\sqrt{\sigma_{I N C_{2}} \sigma_{E X C_{\imath}}},
$$

which provides an estimate of the point resolution. 


\section{References}

[1] ILC Collaboration, Internation Linear Collider: Reference Design Report (2007) URL http://www.linearcollider.org/about/Publications/ Reference-Design-Report [Retrieved: May 19, 2011]

[2] ILD Concept Group, The International Large Detector: Letter of Intent (2010). URL http://arxiv.org/abs/1006.3396v1 [Retrieved: May 19, 2011]

[3] D.R. Nygren. A time projection chamber. Presented at 1975 PEP Summer Study, PEP 198 (1975) and included in Proceedings.

[4] R.J. Fernow. Introductıon to Experımental Partıcle Physıcs. Cambridge University Press (1986).

[5] Particle Data Group, Review of particle physics. Phys. Lett. B, 667(1), (2008).

[6] Magboltz 2, version 8.6 (2009) CERN library. Also see: S. Biagi. Monte Carlo simulation of electron drift and diffusion in counting gases under the influence of electric and magnetic fields. Nucl. Instr. and Meth. in Phys. Res. A 421 (1999) 234-240.

[7] W.B. Atwood et al. Performance of the ALPEH Time Projection Chamber. Nucl. Instr. Meth. in Phys. Res. A 306 (1991) 446-458.

[8] F. Sauli. GEM: A new concept for electron amplification in gas detectors. Nucl. Instr. and Meth. in Phys. Res. A 386 (1997) 531-534

[9] GAS DETECTORS DEVELOPMENT - CERN URL http://gdd. web.cern . ch/GDD [Retrieved: May 19, 2011]

[10] G. Rosenbaum. Measuring the Resolution of a GEM - TPC in a Magnetic Field. MSc Thesis, University of Victoria (2005). URL http://www-flc. desy.de/tpc/documents/theses.php [Retrieved: May 19, 2011] 
[11] I. Giomataris. Development and prospects of the new gaseous detector Micromegas. Nucl. Instr. and Meth. in Phys. Res. A 419 (1998) 239-250.

[12] I. Giomataris. MICROMEGAS: results and prospects. URL http: //www.slac.stanford.edu/pubs/icfa/fal199/paper1/paper1.pdf [Retrieved: May 19, 2011]

[13] M.S. Dixit and A. Rankin. Simulating the charge dispersion phenomena in Micro-Pattern Gas Detectors with a resistive anode. Nucl. Instr. Meth. in Phys. Res. A 566 (2006) 281-285.

[14] M. Dixit, J. Dubeau, J.-P. Martin, and K. Sachs. Position Sensing from charge dispersion in micro-pattern gas detectors with a resistive anode. Nucl. Instr. Meth. in Phys. Res. A 518 (2004) 721-727.

[15] A. Yamamoto et al. BESS-Polar: long duration flights at Antarctica to search for primordial antiparticles. Nuclear Physics B 113 (2002) 208-212.

[16] K. Boudjemline et al. Resolution studies in Micromegas-TPC using charge dispersion in a magnetic field. Presented at IEEE NSS/MIC Conf, San Diego, California, Oct. 2006.

[17] M. Dixit, D. Attie, A. Bellerive, K. Boudjemline, P. Colas, P. Giganon, I. Giomataris, V. Lepeltier, S. Liu, J.-P. Martin, K. Sachs, Y. Shin, and S. Turnbull. Micromegas TPC studies at high magnetic field using the charge dispersion signal. Nucl. Instr. and Meth. in Phys. Res. A 581 (2007) 254-257.

[18] R. Brun and F. Rademakers. ROOT - An Object Oriented Data Analysis Framework, Nucl. Instr. Meth. in Phys. Res. A389 (1997) 81-86. URL http: //root.cern.ch [Retrieved: May 19, 2011]

[19] R.K Carnegie, M.S. Dixit, J. Dubeau, D. Karlen, J.-P. Martin, H. Mes, and K. Sachs. Resolution studies of cosmic-ray tracks in a TPC with GEM readout. Nucl. Instr. Meth. in Phys. Res. A 538 (2005) 372-383.

[20] W. Blum, W. Riegler, L. Rolandi. Partıcle Detectıon with Drift Chambers, 2nd Edıtıon. Springer (2008).

[21] J.H. Thomas et al. The STAR time projection chamber: a unique tool for studying high multiplicity events at RHIC. Nucl. Instr. Meth. in Phys. Res. A 499 (2003) 659-678. 
[22] J. R. Taylor. An Introduction to Error Analysıs, 2nd Edıtıon. University Science Books (1997)

[23] J. Stewart. Calculus, 4th Edıtıon. Brooks/Cole Publishing Company (1999)

[24] J.F. Epperson. An Introduction to Numerical Methods and Analysis, Revised Edıtıon. Wiley (2007)

[25] F.B. Hildebrand. Introductıon to Numerical Analysıs, 2nd Edıtıon. Dover Publications (1987) 